Portland State University

PDXScholar

6-2-1993

\title{
Measurement Techniques for Noise Figure and Gain of Bipolar Transistors
}

Wayne Kan Jung

Portland State University

Follow this and additional works at: https://pdxscholar.library.pdx.edu/open_access_etds

Part of the Electrical and Computer Engineering Commons Let us know how access to this document benefits you.

Recommended Citation

Jung, Wayne Kan, "Measurement Techniques for Noise Figure and Gain of Bipolar Transistors" (1993). Dissertations and Theses. Paper 4592.

https://doi.org/10.15760/etd.6476

This Thesis is brought to you for free and open access. It has been accepted for inclusion in Dissertations and Theses by an authorized administrator of PDXScholar. Please contact us if we can make this document more accessible: pdxscholar@pdx.edu. 
AN ABSTRACT OF THE THESIS OF Wayne Kan Jung for the Master of Science in Electrical and Computer Engineering presented June 2, 1993.

Title: Measurement Techniques for Noise Figure and Gain of Bipolar Transistors.

\section{APPROVED BY THE MEMBERS OF THE THESIS COMMITTEE:}

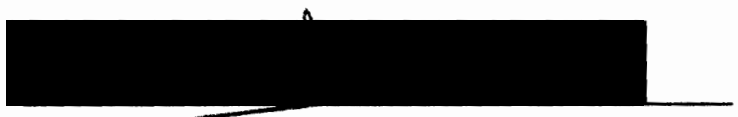

Paul Van Halen, Chair

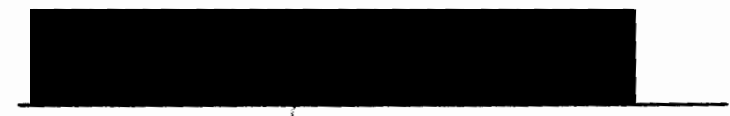

Branimir Pejcinovic

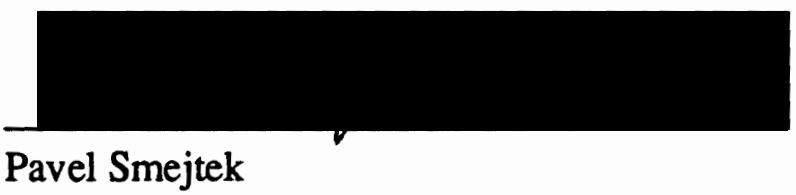

First, the concepts of reflection coefficients, s-parameters, Smith chart, noise figure, and available power gain will be introduced. This lays out the foundation for the presentation of techniques on measuring noise figure and gain of high speed bipolar junction transistors. Noise sources in a bipolar junction transistor and an equivalent circuit including these noise sources will be presented. The process of determining the noise parameters of a transistor will also be discussed. A Pascal program and several TEKSPICE scripts are developed to calculate the stability, available power gain, and noise figure circles. Finally, these circles are plotted on a Smith chart to give a clear view of how a transistor will perform due to a change in source impedances. 


\title{
MEASUREMENT TECHNIQUES FOR NOISE FIGURE AND GAIN OF BIPOLAR TRANSISTORS
}

\author{
by \\ WAYNE KAN JUNG
}

A thesis submitted in partial fulfillment of the requirements for the degree of

\author{
MASTER OF SCIENCE \\ in \\ ELECTRICAL AND COMPUTER ENGINEERING
}

Portland State University

1993 


\section{TO THE OFFICE OF GRADUATE STUDIES:}

The members of the Committee approve the thesis of Wayne Kan Jung presented June 2, 1993.

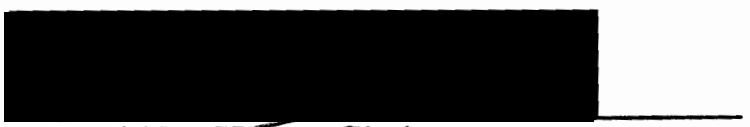

Paul Van Hăen, Chair

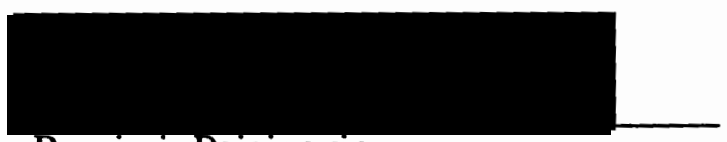

Branimir Pejcinovic

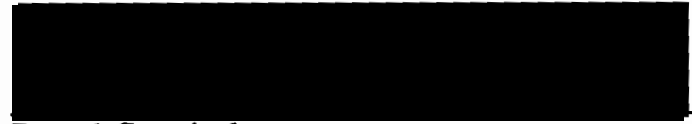

Pavel Smejtek

APPROVED:
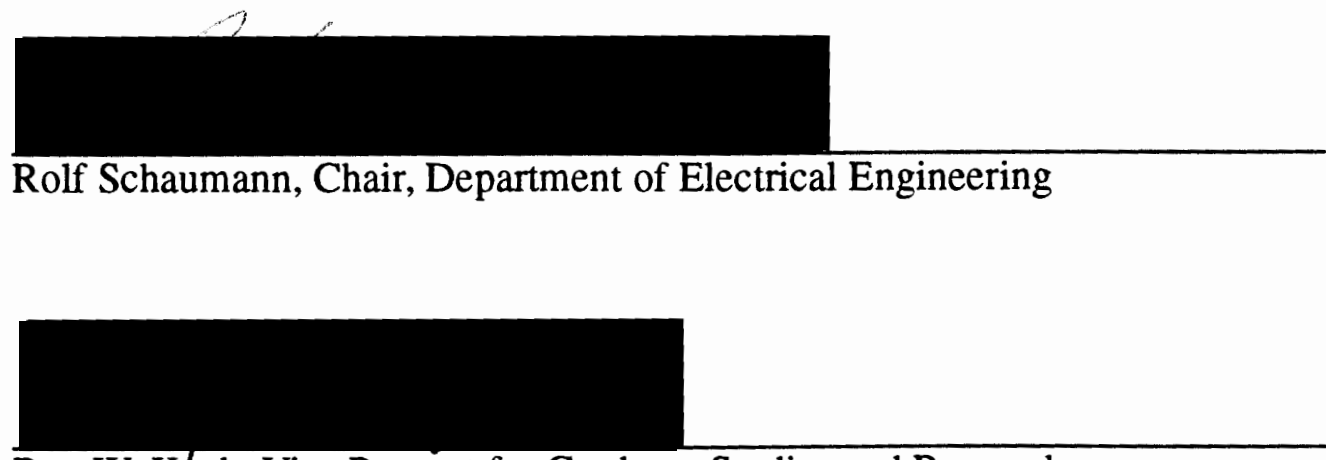

Roy W. Kpch, Vice Provost for Graduate Studies and Research 


\section{ACKNOWLEDGEMENTS}

I would like to take this opportunity to thank those who help make thesis possible. First, I deeply appreciate my academic advisor Dr. Paul Van Halen for his guidance and support throughout the completion of this thesis. Second, I thank my mentor John Brewer, manager of Wireless Telecommunication Product Group of Tektronix' Micro-Product Line, and other engineers from Tektronix: James Marsh, Ken Weigel, Shaun Simpkins, and Tim Davis, for their guidance and support for completing this work. Finally, special thanks are due to Dr. Branimir Pejcinovic and Dr. Pavel Smejtek of Portland State University for their interest and support. 


\section{TABLE OF CONTENTS}

PAGE

ACKNOWLEDGEMENTS $\ldots \ldots \ldots \ldots \ldots \ldots \ldots \ldots \ldots \ldots \ldots \ldots \ldots \ldots$ ii

LIST OF TABLES $\ldots \ldots \ldots \ldots \ldots \ldots \ldots \ldots \ldots \ldots \ldots \ldots$ vi

LIST OF FIGURES $\ldots \ldots \ldots \ldots \ldots \ldots \ldots \ldots \ldots \ldots \ldots \ldots \ldots \ldots \ldots \ldots \ldots$

CHAPTER

I INTRODUCTION $\ldots \ldots \ldots \ldots \ldots \ldots \ldots \ldots \ldots \ldots \ldots \ldots$

Purpose of the Analysis $\ldots \ldots \ldots \ldots \ldots \ldots \ldots \ldots 1$

Outcome of the Analysis $\ldots \ldots \ldots \ldots \ldots \ldots \ldots \ldots 2$

II EXPLANATION OF TERMS $\ldots \ldots \ldots \ldots \ldots \ldots \ldots \ldots$

S-parameters $\ldots \ldots \ldots \ldots \ldots \ldots \ldots \ldots \ldots \ldots \ldots$

Introduction

Reflection Coefficients

S-parameters

Smith Chart

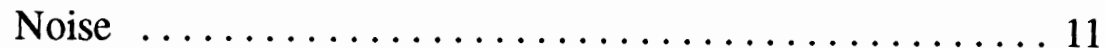

Common Types of Noise

Noise Figure

Available Power Gain $\ldots \ldots \ldots \ldots \ldots \ldots \ldots \ldots \ldots$

III NOISE FIGURE MEASUREMENT $\ldots \ldots \ldots \ldots \ldots \ldots \ldots \ldots$

Noise in Bipolar Transistor $\ldots \ldots \ldots \ldots \ldots \ldots \ldots \ldots$

Flicker Noise

Burst Noise

Shot Noise

Thermal Noise

Equivalent Circuit 
Determination of Noise Parameters $\ldots \ldots \ldots \ldots \ldots \ldots 20$

Definition

Source Impedance Selections

Noise Figure Measurement from TEKSPICE

Noise Parameters Computation

Noise Figure Circles $\ldots \ldots \ldots \ldots \ldots \ldots \ldots \ldots 28$

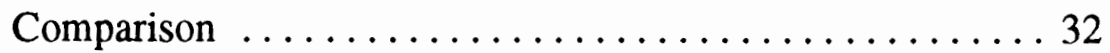

IV GAIN MEASUREMENT $\ldots \ldots \ldots \ldots \ldots \ldots \ldots \ldots \ldots \ldots$

Gain and Stability $\ldots \ldots \ldots \ldots \ldots \ldots \ldots \ldots \ldots \ldots \ldots$

Stability Criterion

Stability Circles

Gain Circles

S-parameters from TEKSPICE $\ldots \ldots \ldots \ldots \ldots \ldots \ldots 42$

Comparison $\ldots \ldots \ldots \ldots \ldots \ldots \ldots \ldots \ldots \ldots \ldots$

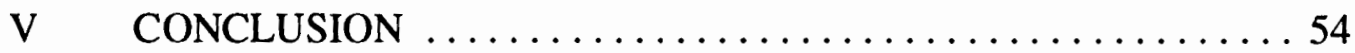

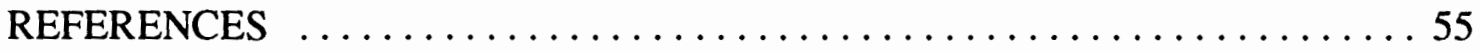

APPENDICES

A CONSTRUCTION AND APPLICATION OF

SMITH CHART $\ldots \ldots \ldots \ldots \ldots \ldots \ldots \ldots \ldots \ldots$

B NOISE FIGURE CALCULATIONS $\ldots \ldots \ldots \ldots \ldots \ldots \ldots \ldots$

C NOISE PARAMETERS AND NOISE FIGURE CIRCLES

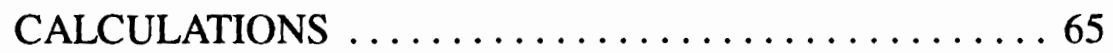

D STABILITY CIRCLES COMPUTATIONS $\ldots \ldots \ldots \ldots \ldots 75$

E GAIN CIRCLES COMPUTATIONS $\ldots \ldots \ldots \ldots \ldots \ldots \ldots$

F S-PARAMETERS EXTRACTION $\ldots \ldots \ldots \ldots \ldots \ldots \ldots \ldots 2$ 


\section{LIST OF TABLES}

TABLE

PAGE

I. Noise Figure Measured at Various Source Impedances

with $\mathrm{V}_{\mathrm{BE}}=0.8 \mathrm{~V}, \mathrm{~V}_{\mathrm{CE}}=4 \mathrm{~V}$ at Frequency $=1 \mathrm{GHz} \ldots \ldots 24$

II. Noise Parameters of N16 and G14V102 Transistors ........ 28 


\section{LIST OF FIGURES}

FIGURE

PAGE

1. A General Two-port Network $\ldots \ldots \ldots \ldots \ldots \ldots \ldots \ldots$

2. Traveling Waves $\ldots \ldots \ldots \ldots \ldots \ldots \ldots \ldots \ldots \ldots \ldots \ldots \ldots \ldots \ldots$

3. Incident and Reflected Waves in a Two-port Network $\ldots \ldots \ldots 8$

4. The Smith Chart $\ldots \ldots \ldots \ldots \ldots \ldots \ldots \ldots \ldots \ldots \ldots$

5. Noise Figure of Network in Cascade $\ldots \ldots \ldots \ldots \ldots \ldots \ldots$

6. Available Power Gain of a Two-port Network $\ldots \ldots \ldots \ldots 14$

7. Bipolar Transistor Small-signal Equivalent Circuit with Noise Sources $\ldots \ldots \ldots \ldots \ldots \ldots \ldots$

8. Noise Figure vs. Frequency for N16 ............. 19

9. Parabolic Noise Surface $\ldots \ldots \ldots \ldots \ldots \ldots \ldots \ldots \ldots \ldots$

10. Test Circuit for Noise Figure $\ldots \ldots \ldots \ldots \ldots \ldots \ldots \ldots \ldots$

11. Noiseless Resistor Model .................. 24

12. Constant Noise Figure Circles for N16 with

$$
\mathrm{V}_{\mathrm{BE}}=0.8 \mathrm{~V}, \mathrm{~V}_{\mathrm{CE}}=4 \mathrm{~V} \text { at Frequency }=1 \mathrm{GHz} \ldots \ldots \ldots \ldots . \ldots 31
$$

13. Constant Noise Figure Circles for N16 with

$\mathrm{V}_{\mathrm{BE}}=0.76 \mathrm{~V}, \mathrm{~V}_{\mathrm{CE}}=4 \mathrm{~V}$ at Frequency $=900 \mathrm{MHz} \ldots \ldots . \ldots 33$

14. Constant Noise Figure Circles for G14V102 with

$\mathrm{V}_{\mathrm{BE}}=0.76 \mathrm{~V}, \mathrm{~V}_{\mathrm{CE}}=4 \mathrm{~V}$ at Frequency $=900 \mathrm{MHz} \ldots \ldots 34$

15. Constant Noise Figure Circles for N16 with

$\mathrm{V}_{\mathrm{BE}}=0.76 \mathrm{~V}, \mathrm{~V}_{\mathrm{CE}}=4 \mathrm{~V}$ at Frequency $=1.6 \mathrm{GHz} \ldots \ldots .35$ 
16. Constant Noise Figure Circles for G14V102 with

$$
\mathrm{V}_{\mathrm{BE}}=0.76 \mathrm{~V}, \mathrm{~V}_{\mathrm{CE}}=4 \mathrm{~V} \text { at Frequency }=1.6 \mathrm{GHz} \ldots \ldots . .36
$$

17. Common Emitter Amplifier with Local Series Feedback ... . . 37

18. Stability Circles Construction on Smith Chart $\ldots \ldots \ldots \ldots . .40$

19. Stability Region for $\Gamma_{s} \ldots \ldots \ldots \ldots \ldots \ldots \ldots \ldots \ldots \ldots$

20. Test Circuit for Obtaining $S_{11}$ and $S_{21} \ldots \ldots \ldots \ldots \ldots$

21. Test Circuit for Obtaining $S_{22}$ and $S_{12} \ldots \ldots \ldots \ldots \ldots$

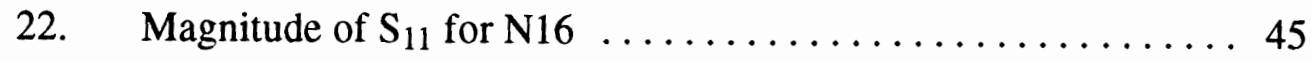

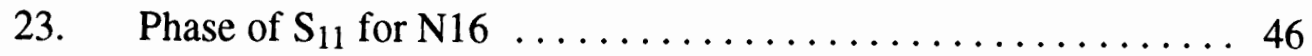

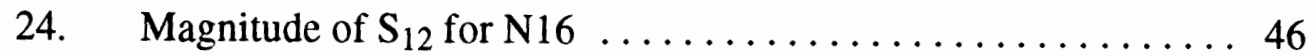

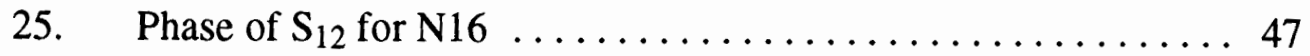

26. Magnitude of $S_{21}$ for $\mathrm{N} 16 \ldots \ldots \ldots \ldots \ldots \ldots \ldots \ldots \ldots$

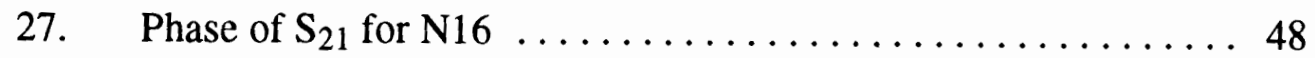

28. Magnitude of $S_{22}$ for $N 16 \ldots \ldots \ldots \ldots \ldots \ldots \ldots \ldots$

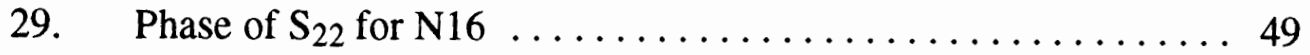

30. Gain and Stability Circles for N16 at

Frequency $=900 \mathrm{MHz} \ldots \ldots \ldots \ldots \ldots \ldots \ldots \ldots$

31. Gain and Stability Circles for G14V102 at

Frequency $=900 \mathrm{MHz} \ldots \ldots \ldots \ldots \ldots \ldots \ldots \ldots \ldots \ldots \ldots$

32. Gain and Noise Figure Circles for N16 at

Frequency $=900 \mathrm{MHz} \ldots \ldots \ldots \ldots \ldots \ldots \ldots \ldots \ldots \ldots \ldots \ldots \ldots$

33. Gain and Noise Figure Circles for G14V102 at

Frequency $=900 \mathrm{MHz} \ldots \ldots \ldots \ldots \ldots \ldots \ldots \ldots \ldots \ldots$

34. Smith Chart Construction $\ldots \ldots \ldots \ldots \ldots \ldots \ldots \ldots$ 
35. A Complete Smith Chart $\ldots \ldots \ldots \ldots \ldots \ldots \ldots \ldots \ldots$

36. Flow Chart for Noise Parameters Calculation ........... 64 


\section{CHAPTER I}

\section{INTRODUCTION}

In all things on earth there exists a degree of randomness. In the world of electronics, the random nature of electric current has been known for over a hundred years. It is a fact that no electronic system is completely free of random noise. Small voltage fluctuations due to noise are always occurring in electronic circuits because electrons are discrete and are constantly moving in time. The term "noise" originated with the study of high-gain audio-frequency amplifiers. When a fluctuating voltage or current generated in a device is amplified by an audio-frequency amplifier and the amplified signal is fed into a loudspeaker, the loudspeaker produces a hissing sound, hence the name noise. This descriptive term, noise, now refers to any spontaneous fluctuation in a system, regardless of whether an audible sound is produced.

\section{PURPOSE OF THE ANALYSIS}

Noise obscures low level electrical signals, therefore it can be a limiting factor on component and system performance. In a spectrum analyzer, for example, noise limits the sensitivity of the instrument-that is, the lowest-amplitude signal that the analyzer can detect and display. In a radar, noise can obscure returns from a target, and limit the effective range of the radar. In digital communications, excessive noise can cause a high Bit-Error-Rate, resulting in the transmission and reception of false information.

As the demands for portable personal communication system have skyrocketed in recent years, more delicate signal receivers are needed to pick up small signals from miniature portable transmitters. How small can the signal be and still be detected 
without severe degradation in signal quality? It depends on the noise immunity of the receiver, which in turn depends on the noise performance of the components inside the receiver. Receivers with high noise immunity require less powerful transmitters. Reduced transmitter power will lower the size and cost of the transmitter. This is highly desirable for low power units, such as hand-held mobile phones, portable Global Positioning Systems (GPS), and Personal Communication Networks (PCN) where size and battery life form constraints on the system.

\section{OUTCOME OF THE ANALYSIS}

This analysis studies the noise and gain characteristics of the basic component in an amplifier block, the transistor, in the microwave frequency range. After identifying the noise sources in the transistor, the effect of each noise source on the performance of the device is discussed. This study will provide spot noise figure data for two high speed silicon bipolar transistors manufactured on two processes from Tektronix' Microelectronic Operation (SHPi and GST-1). SHPi is Tektronix' latest generation Super-High frequency bipolar processes. The $f_{T}$ of the transistor of interest (N16) of this process is measured to be close to $9 \mathrm{GHz}$ under the condition: $I_{C}=9 \mathrm{~mA}$ and $V_{C E}=4 \mathrm{~V}$. GST-1 (Giga-Speed Si-Bipolar Technology) is a high speed self-aligned double-polysilicon process. GST-1 is designed for the purpose of building high density, high performance circuits. The $f_{T}$ for the G14V102, an N16 equivalent in the GST-1 process, is in the proximity of $12 \mathrm{GHz}$ with $I_{C}=25 \mathrm{~mA}$ and $V_{C E}=4 \mathrm{~V}$. A systematic way to measure noise figure is presented. Noise figure data are processed by a computer program to find the minimum noise figures and the coordinates of noise figure circles on a Smith chart. TEKSPICE (An enhanced version of SPICE developed by engineers at Tektronix, Inc.) scripts are also presented for s-parameter extraction, stability circles and available power gain circles calculation of bipolar transistors. Finally, the two most 
important parameters in microwave design (available power gain and noise figure) are plotted on a Smith chart to give a clear view of how a particular device will perform in various source impedances. 


\section{CHAPTER II}

\section{EXPLANATION OF TERMS}

The measurement techniques used at microwave frequencies are different compared with low frequency methods. At lower frequencies, the properties of a circuit or system are determined by measuring voltages and currents. This approach is not applicable to microwave circuits since oftentimes these quantities are not uniquely defined. As a result, most microwave experimentation involves the accurate measurement of impedance and power rather than voltage and current. In this chapter, some basic concepts involved in microwave circuit design will be explained. They are: reflection coefficients, scattering parameters, Smith chart, noise figure, and available power gain.

\section{S-PARAMETERS}

\section{Introduction}

Linear networks can be completely characterized by parameters measured at the network terminals without regard to the contents of the network. Once the parameters of a network have been determined, its behavior in any external environment can be predicted, again without regard to the specific contents of the network.

A two-port device can be described by a number of parameter sets. Hybrid, admittance, and impedance parameters sets are often used at low frequency analysis. They are abbreviated to $\mathrm{H}, \mathrm{Y}$, and Z-parameters respectively. They relate the four variables of a two-port $\left(V_{1}, I_{1}, V_{2}\right.$, and $\left.I_{2}\right)$ in a similar way, the only difference in the 
parameter sets is the choice of independent and dependent variables. The parameters sets

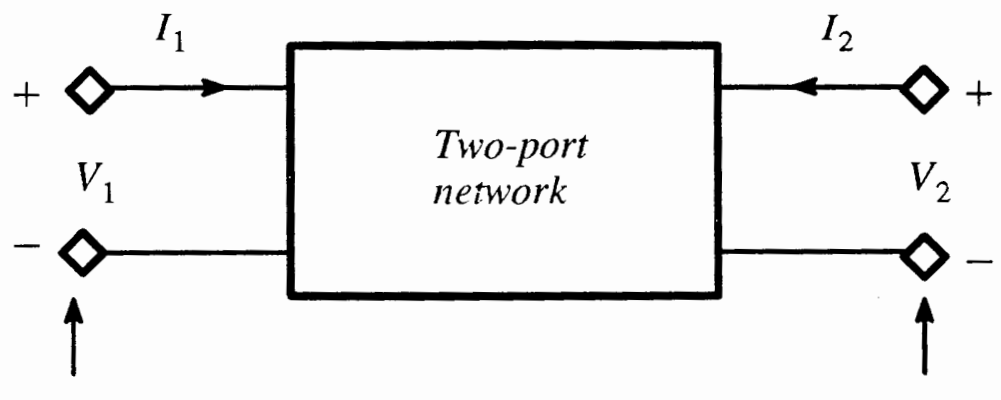

Port 1

Port 2

Figure 1. A general two-port network.

are defined as

\section{H-Parameters}

$$
\begin{aligned}
& V_{1}=h_{11} I_{1}+h_{12} V_{2} \\
& I_{2}=h_{21} I_{1}+h_{22} V_{2}
\end{aligned}
$$

Y-Parameters

$$
\begin{aligned}
& I_{1}=y_{11} V_{1}+y_{12} V_{2} \\
& I_{2}=y_{21} V_{1}+y_{22} V_{2}
\end{aligned}
$$

\section{Z-Parameters}

$$
\begin{aligned}
& V_{1}=z_{11} I_{1}+z_{12} I_{2} \\
& V_{2}=z_{21} I_{1}+z_{22} I_{2}
\end{aligned}
$$

Moving to higher frequencies, some problems arise:

1. Equipment is not readily available to measure total voltage and total current at the ports of the network. The voltage measured will not be the same as the voltage at the ports of the network if the length of the transmission line is comparable with the wavelength of the test signal.

2. Active devices, such as transistors, very often will not be short or open circuit stable. 
3. Parasitics in active devices may cause unwanted oscillations.

Some other sets of parameters are necessary to overcome these problems. Hence, dissipating (resistive) loads are used in measurements to minimize parasitic oscillations. In addition, the concept of traveling waves rather than total voltages and currents is used to describe the networks. Voltage, current, and power can be considered to be in the form of waves traveling in both directions along a transmission line (Fig. 2). A portion

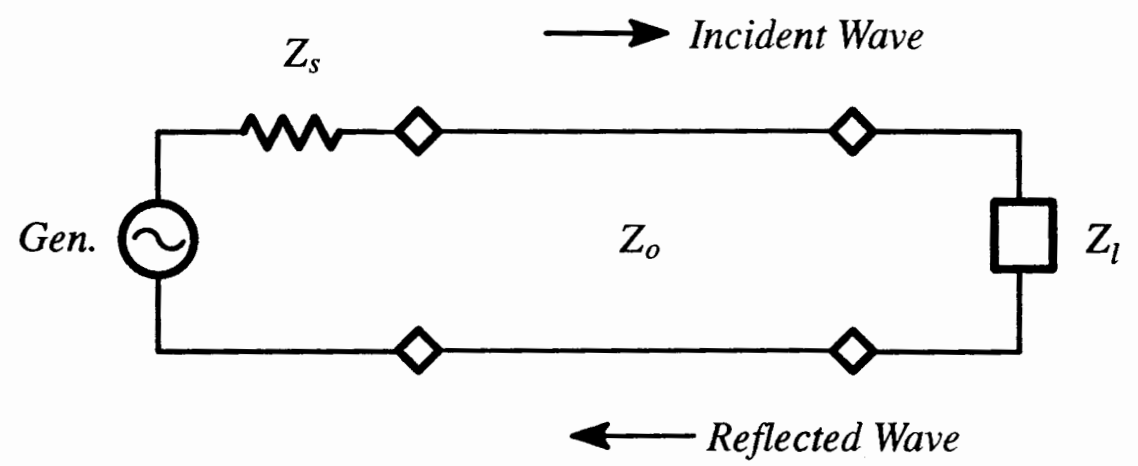

Figure 2. Traveling waves.

of the waves incident on the load will be reflected if the load impedance $Z_{l}$ is not equal to the characteristic impedance of the transmission line $Z_{o}$. This is analogous to the concept of maximum power transfer. A source will deliver maximum power to a load (no reflection from the load) if the load impedance is equal to the source impedance.

\section{$\underline{\text { Reflection Coefficients }}$}

The reflection coefficient Eq. (1) is a mathematical representation of the reflected voltage wave with respect to the incident voltage wave at a specified port of a circuit.

$$
\Gamma=\frac{\text { Reflected Wave }}{\text { Incident Wave }}
$$

Eq. (2) defines the load reflection coefficient in terms of the load impedance referenced to the characteristic impedance of the transmission line.

$$
\Gamma_{l}=\frac{Z_{l}-Z_{o}}{Z_{l}+Z_{o}}
$$


And similarly the source reflection coefficient is

$$
\Gamma_{s}=\frac{Z_{s}-Z_{o}}{Z_{s}+Z_{o}}
$$

It is well known that maximum power transfer occurs when the impedance of the source matches the impedance of the load. For $\Gamma=0$, maximum power transfer occurs, and $\Gamma$ equals unity when there is no power transfer since then all the incident power is reflected back.

To facilitate computations, the source impedance and the load impedance are normalized to the characteristic impedance of the transmission line, $\bar{Z}_{l}=\frac{Z_{l}}{Z_{o}}$. Expressed in terms of normalized impedances, the reflection coefficients at the load and at the source are

$$
\Gamma_{l}=\frac{\bar{Z}_{l}-1}{\bar{Z}_{l}+1}
$$

and

$$
\Gamma_{s}=\frac{\bar{Z}_{s}-1}{\bar{Z}_{s}+1}
$$

\section{S-parameters}

For a two-port network shown in Fig. 3, the following new variables are defined [1]:

$$
\begin{aligned}
& a_{1}=\frac{E_{i 1}}{\sqrt{Z_{o}}} \\
& a_{2}=\frac{E_{i 2}}{\sqrt{Z_{o}}} \\
& b_{1}=\frac{E_{r 1}}{\sqrt{Z_{o}}} \\
& b_{2}=\frac{E_{r 2}}{\sqrt{Z_{o}}}
\end{aligned}
$$




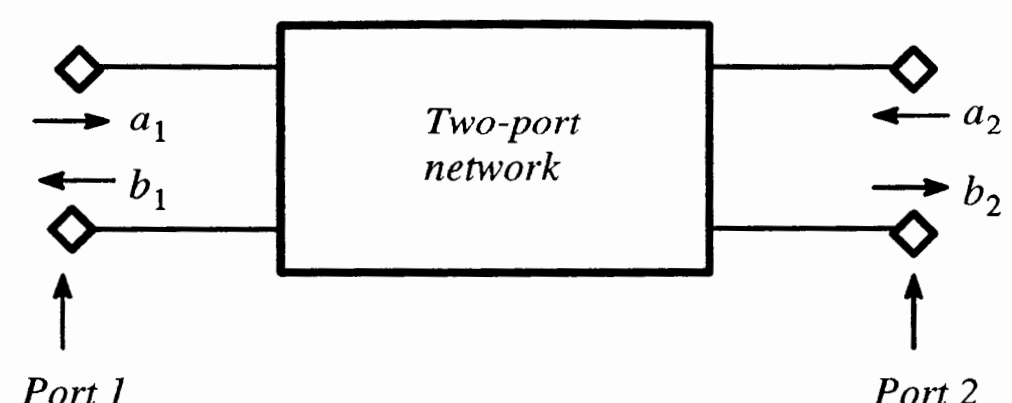

Figure 3. Incident and reflected waves in a two-port network.

where $E_{i}$ and $E_{r}$ are the incident voltage wave and reflected voltage wave respectively. Notice that the square of the magnitude of these new variables has the dimension of power. By measuring incident and reflected power from a two-port network, we avoid the primary problem in microwave measurement, which is the voltage variation along the line between the device under test and the test equipment. A new set of parameters relate these four waves in the following fashion:

$$
\begin{aligned}
& b_{1}=S_{11} a_{1}+S_{12} a_{2} \\
& b_{2}=S_{21} a_{1}+S_{22} a_{2}
\end{aligned}
$$

where

$$
\begin{aligned}
& S_{11}=\left.\frac{b_{1}}{a_{1}}\right|_{a_{2}=0} \quad \text { input reflection coefficient with the output matched } \\
& S_{21}=\left.\frac{b_{2}}{a_{1}}\right|_{a_{2}=0} \begin{array}{l}
\text { forward transmission coefficient with the output } \\
\text { matched }
\end{array} \\
& S_{22}=\left.\frac{b_{2}}{a_{2}}\right|_{a_{1}=0} \quad \text { output reflection coefficient with the input matched } \\
& S_{12}=\left.\frac{b_{1}}{a_{2}}\right|_{a_{1}=0} \begin{array}{l}
\text { reverse transmission coefficient with the input } \\
\text { matched }
\end{array}
\end{aligned}
$$

This set of new parameters is called "scattering parameters," since they relate those waves scattered or reflected from the network to those waves incident upon the 
network. These scattering parameters are commonly referred to as s-parameters.

Although s-parameters completely characterize a linear network, sources and loads need to be attached before the network can be used. The new input reflection coefficient $\Gamma_{\text {in }}$ an the new output reflection coefficient $\Gamma_{\text {out }}$ take the form [2]:

$$
\Gamma_{\text {in }}=S_{11}+\frac{S_{12} S_{21} \Gamma_{l}}{1-S_{22} \Gamma_{l}}
$$

and

$$
\Gamma_{\text {out }}=S_{22}+\frac{S_{12} S_{21} \Gamma_{s}}{1-S_{11} \Gamma_{s}}
$$

The second terms in the above equations show the interaction between the two ports. If $S_{12}$ is equal to zero, the two-port network is called unilateral because whatever happens to one port does not affect the other port.

For a bilateral linear two-port network, the input reflection coefficient is not just function of $S_{11}$. It is a function of $\Gamma_{l}$ as well. Therefore, the effect of the load must be considered when an input match circuit is built to match the signal source. Also, $\Gamma_{\text {out }}$ is a function of the s-parameters and $\Gamma_{s}$, and this should be kept in mind when an output matching network is built to have all the power delivered to the load.

\section{Smith Chart}

The Smith chart (Fig. 4) is a design and analysis tool that can provide insight into the impedance and reflection characteristics of a circuit. The chart is a graphical representation that provides a transformation between the complex reflection coefficient in a polar format to the real and imaginary parts of the impedance, Eq. (2) and Eq. (3). The chart has the property of being able to graphically display the entire range of real and-imaginary values of input impedances of a network. Therefore, on a Smith chart a point simultaneously represents three different things. Depending on the coordinate system used as a reference, they are: reflection coefficient, impedance, and admittance. 
Impedance matching circuits can be easily and quickly designed using the Smith chart. The chart is also used to present the source impedance dependence of noise figure and power gain of microwave amplifiers. A description on the construction and usage of a Smith chart is presented in Appendix A.

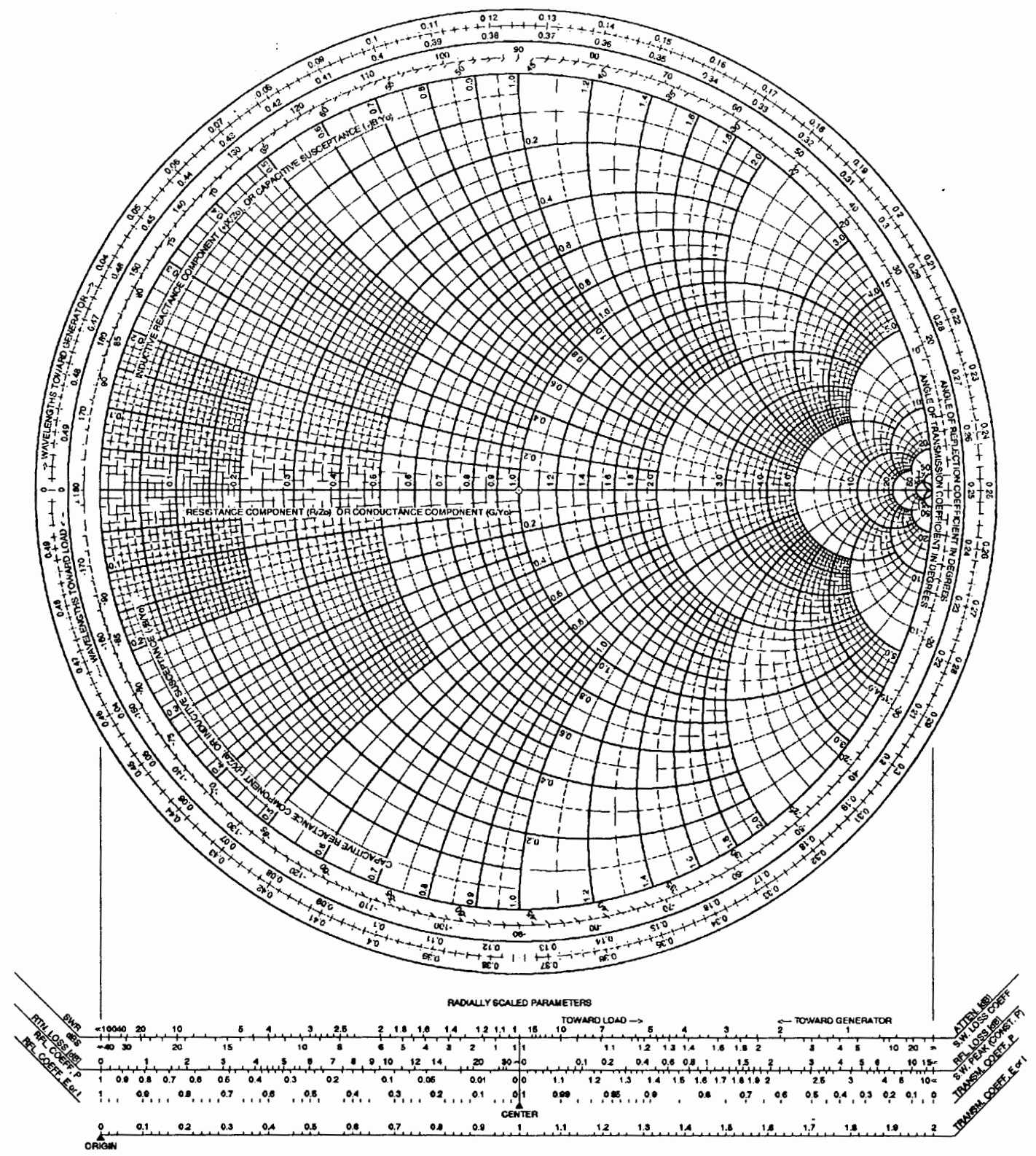

Figure 4. The Smith chart. 


\section{NOISE}

\section{Common Types of Noise}

Any undesired signals present in signal processing systems and their components are called electrical noise. Common types of electrical noise occurring in nature are impulse noise, quantizing noise, thermal noise, and shot noise.

Impulse noise is generated by e.g. automobile ignition, lightning in the atmosphere, neon signs, and power-line hum. As the name implies, these noises are occurring as impulses in the frequency spectrum. Power-line hum constitutes a spike at $60 \mathrm{~Hz}$, and automobile ignition may produce spikes from several hundred hertz to several thousand hertz. This type of noise can be reduced or eliminated by proper shielding of the system.

Quantizing noise is usually associated with digital systems, and their interfaces with the outside world. It is caused by sampling amplitude errors in digitizing systems. Errors introduced by digital to analog converter and analog to digital converter are the best examples of quantizing noise. This noise can be reduced to a negligible level by using more discrete levels to represent the continuous analog signal, but it can not be eliminated completely.

Thermal noise is caused by the thermal agitation of electrons. As the name implies, the amount of thermal noise is proportional to the temperature of a conducting device. With temperature above $O K$, electrons have sufficient energy to move within a conductor in all directions. Due to the randomness of electron motion, the voltage across the device or the current through the device fluctuates about an average value. Because its power spectral density is constant over frequency thermal noise is also called white noise.

Shot noise originates in the discrete nature of electron flow. Current is defined as change of charge $d q$ passes through any cross section of the conductor in an infinitesimal 
interval of time $d t$.

$$
i=\frac{d q}{d t}
$$

The number of electrons crossing at time interval $d t_{1}$ will be slightly different from the number of electrons crossing at time interval $d t_{2}$. These differences constitute fluctuations in current, and shot noise is a measure of these fluctuations. In semiconductors, shot noise is due to the electron flow across junctions. Like thermal noise, it also has uniform spectral density at all frequencies.

Impulse noise and quantizing noise are man-made and can be controlled. The amount of thermal noise is proportional to the temperature and the resistance of the device, therefore it is always present in a system because the operating temperature is not at absolute zero nor are there perfect conductors. The last type of electrical noise, shot noise is basically due to the fact that electrical charge is not continuous but is carried in discrete amounts equal to the electron charge. Noise is thus associated with fundamental processes in the integrated circuit devices.

\section{Noise Figure}

To compare the performance of high frequency devices, an important figure of merit is the noise factor/figure, which is a measure of how the thermal noise and the shot noise generated in semiconductors degrade the signal-to-noise ratio. The noise factor $F$ of the network is defined as the ratio of the available signal-to-noise ratio at the signal generator terminals to the available signal-to-noise ratio at its output terminals.

$$
F=\frac{S_{i} / N_{i}}{S_{o} / N_{o}}
$$

The current definition for noise factor applies whether it is linear or logarithmic. The term "noise factor" is used when referring to the ratio in linear terms. The term "noise figure" is used when referring to the ratio in logarithmic terms. The relationship 
for noise factor $(F)$ and noise figure $(N F)$ is shown in the following equation:

$$
N F=10 \log _{10} F
$$

The term noise figure $(N F)$ is more commonly used because signal-to-noise ratios are often expressed in logarithmic forms. A simple subtraction of two signal-to-noise ratios in logarithmic form will give us noise figure.

$$
N F=10 \log _{10} \frac{S_{i}}{N_{i}}-10 \log _{10} \frac{S_{o}}{N_{o}}
$$

From the definition in Eq. (13) the ideal noise factor is unity or noise figure is $0 \mathrm{~dB}$, where there is no degradation in signal-to-noise ratio after the signal passes through the network.

Noise figure is a parameter that applies both to components and systems. The overall system performance can be predicted from the noise figure of the components that go into it. For a number of networks in cascade, as shown in Fig. 5, the system spot

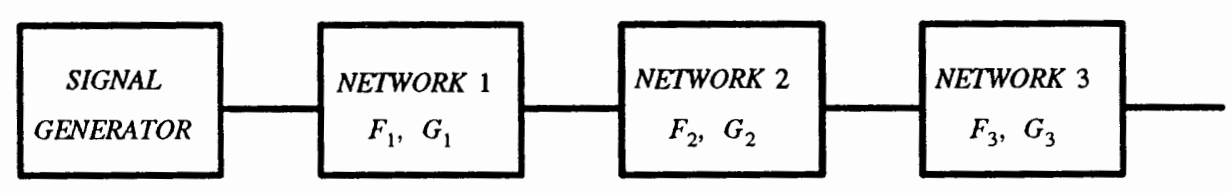

Figure 5. Noise figure of network in cascade.

noise factor is given in terms of the component spot noise factors $\left(F_{i}\right)$ and available gain $\left(G_{i}\right)$ by

$$
F=F_{1}+\frac{F_{2}-1}{G_{1}}+\frac{F_{3}-1}{G_{1} G_{2}}+\cdots
$$

From Eq. (16), the significance of the first stage gain and noise factor are evident. The first stage in the chain has the most significant contribution to the total noise factor of the chain. The total noise factor of the system is at least equal to the noise factor of the first stage. If the first stage gain is significantly large, then the noise contributions from the succeeding stages will be small. 


\section{AVAILABLE POWER GAIN}

A signal generator with an internal impedance $R_{s}$ ohms and output voltage $V_{s}$ volts can deliver $\frac{V_{s}^{2} R_{l}}{\left(R_{s}+R_{l}\right)^{2}}$ watts into a resistance of $R_{l}$ ohms. This power is maximum and equal to $\frac{V_{s}^{2}}{4 R_{s}}$ when the output circuit is matched to the generator impedance, that is when $R_{l}=R_{s}$. Therefore, $\frac{V_{s}^{2}}{4 R_{s}}$ is called the available power of the generator, and it is, by definition, independent of the impedance of the circuit to which it is connected. The output power is smaller than the available power when $R_{l}$ is not equal to $R_{s}$, since there is a mismatch loss. In amplifier input circuits a mismatch condition may be beneficial due to the fact that it may decrease the output noise more than the output signal. It is the presence of such mismatch conditions in amplifier input circuits that makes it desirable to use the term available power.

The symbol $S_{g}$ will be used for the available signal power at the output terminals of the signal generator shown in Fig. 6. The symbol $S$ will be used for the available

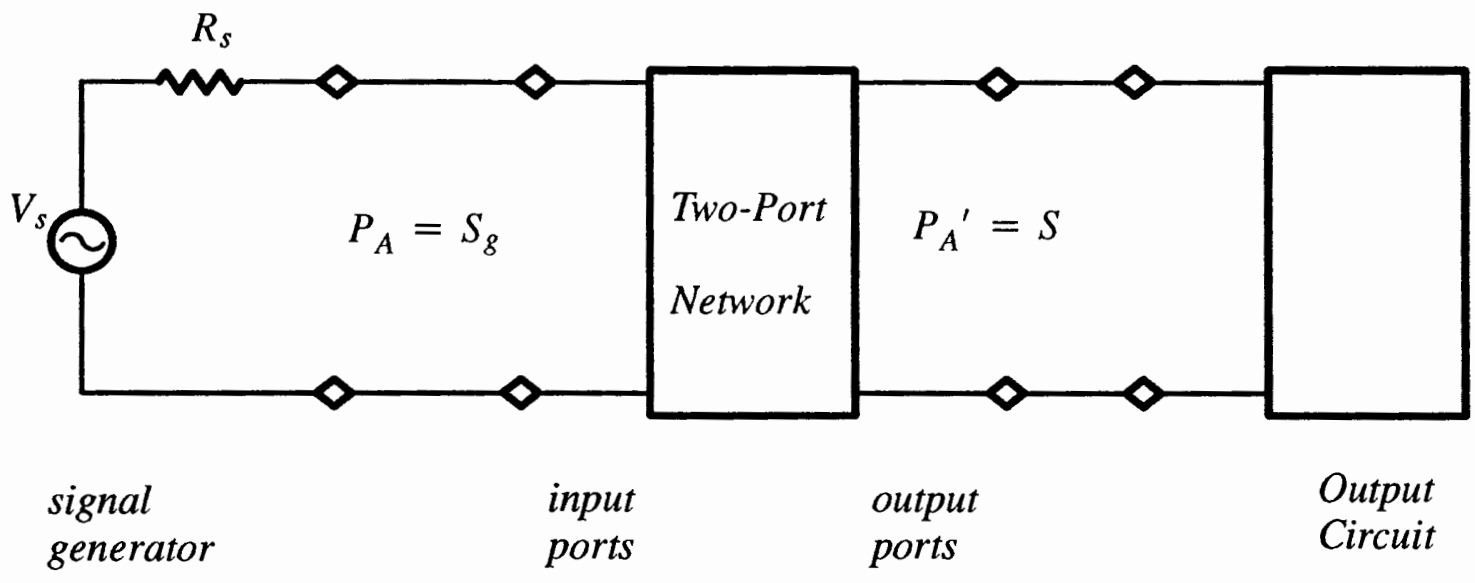

Figure 6. Available power gain of a two-port network.

signal power at the output terminals of the two-port network. 
The gain of the network is defined as the ratio of the available signal power at the output terminals of the network to the available signal power at the output terminals of the signal generator. Hence

$$
G_{A}=\frac{\text { power available from the network }}{\text { power available from the source }}=\frac{S}{S_{8}}
$$

This is an unusual definition of gain since the gain of an amplifier is generally defined as the ratio of its output and input powers. This new definition is introduced here for the same reason that made it desirable to use the term available power. Note that while the gain is independent of the impedance which the output circuit presents to the network, it does depend on the impedance of the signal generator. 


\section{CHAPTER III}

\section{NOISE FIGURE MEASUREMENT}

In this chapter, noise sources inherent in a bipolar transistor will be investigated. The method for obtaining noise parameters from measured or simulated data is presented.

\section{NOISE IN BIPOLAR TRANSISTOR}

The noise sources in a bipolar junction transistor are categorized into four major types:

1. Flicker noise;

2. Burst noise;

3. Shot noise;

4. Thermal noise.

\section{Flicker Noise}

Flicker noise is caused by traps associated with contamination and crystal defects in the emitter-base depletion layer. It is associated with a flow of direct current and displays a spectral density of the form [3]:

$$
\overline{i^{2}}=\frac{K_{F} I^{A_{F}}}{f} \Delta f
$$

where $K_{F}$ is a constant for a particular device, $A_{F}$ is a constant between 0.5 and 2, and $\Delta f$ is the bandwidth in Hertz. This expression shows that the noise spectral density has a $(1 / f)$ frequency dependence, therefore, it is also called $1 / f$ noise. Since flicker noise is most significant at low frequencies, it is not discussed here. 


\section{Burst Noise}

It has been found experimentally that the low frequency noise spectrums of some bipolar transistors show a different frequency dependence than flicker noise. This could be the result of the existence of burst noise. It is caused by the imperfection in the crystal structure. The power spectrum of such signal is given by [4]:

$$
\overline{i^{2}}=\frac{K_{B} I}{1+\left(\frac{\pi f}{2 k}\right)^{2}} \Delta f
$$

where $K_{B}$ is a technological dependent constant for a particular device, and $k$ is the mean repetition rate of the signal. This noise is insignificant at microwave frequencies because it is inversely proportional to $f^{2}$, and it will not be addressed further.

\section{Shot Noise}

Shot noise is due to generation and recombination in the pn junction and injection of carriers across the potential barriers, therefore it is present in all semiconductor diodes and bipolar transistors. Each carrier crosses the junction in a purely random fashion. Thus the current $I$, which appears to be a steady current, is, in fact, composed of a large number of random independent current pulses. The fluctuation in $I$ is termed shot noise and is generally specified in terms of its mean-square variation about the average value $I_{D}$, and it is represented by [3]:

$$
\overline{i^{2}}=2 q I_{D} \Delta f
$$

where $q$ is the electronic charge $\left(1.6 \times 10^{-19} C\right)$. Eq. (21) shows that the noise spectral density is independent of frequency. In a transistor, there are two such noise sources. They are the shot noise in the emitter-base junction $\left(i_{b}\right)$ and in the collector-base junction $\left(i_{c}\right)$. 
Thermal Noise

Thermal noise is due to the random thermal motion of electrons in a resistor, and it is unaffected by the presence or absence of direct current, since typical electron drift velocities in a conductor are much less than electron thermal velocities. As the name indicates, thermal noise is related to absolute temperature $T$.

In a resistor $R$, thermal noise can be represented by a series voltage generator $\overline{v^{2}}$. It is represented by [3]:

$$
\overline{v^{2}}=4 k T R \Delta f
$$

where $k$ is Boltzmann's constant $\left(1.38 \times 10^{-23} \mathrm{~J} / K\right)$, and $T$ is temperature in Kelvin. Like shot noise, thermal noise is also independent of frequency. Thermal noise is a fundamental physical phenomenon and is present in any linear passive resistor.

\section{Equivalent Circuit}

Fig. 7 is the full small-signal equivalent circuit, including noise sources for the bipolar transistor at high frequency [3]. Three noise sources are evident from the figure.

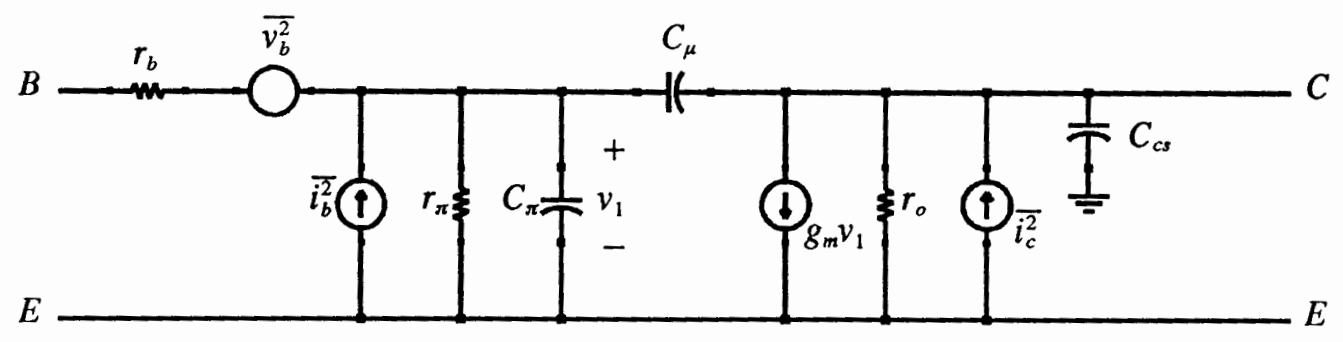

Figure 7. Bipolar transistor small-signal equivalent circuit with noise sources.

They are thermal noise from the series input resistance $\left(r_{b}\right)$ and shot noise due to the base and collector currents $\left(I_{b}, I_{c}\right)$. and their values are:

$$
\begin{aligned}
\overline{v_{b}^{2}} & =4 k T r_{b} \Delta f \\
\overline{i_{b}^{2}} & =2 q I_{b} \Delta f
\end{aligned}
$$




$$
\overline{i_{c}^{2}}=2 q I_{c} \Delta f
$$

The resistors $r_{\pi}$ and $r_{o}$ in Fig. 7 are equivalent circuit elements, not physical resistors, and they do not produce any thermal noise.

Neither thermal noise nor shot noise is frequency dependent, and both exhibit uniform noise output through the entire useful frequency range of the transistor. The internal gain of the transistor does vary with frequency, however, and it falls off as frequency increases. As a result the noise figure begins to rise when the reduction in gain becomes appreciable. Since the power gain falls inversely as frequency squared, the noise figure rises as frequency squared, or $40 d B$ per decade [5]. Fig. 8 graphically shows

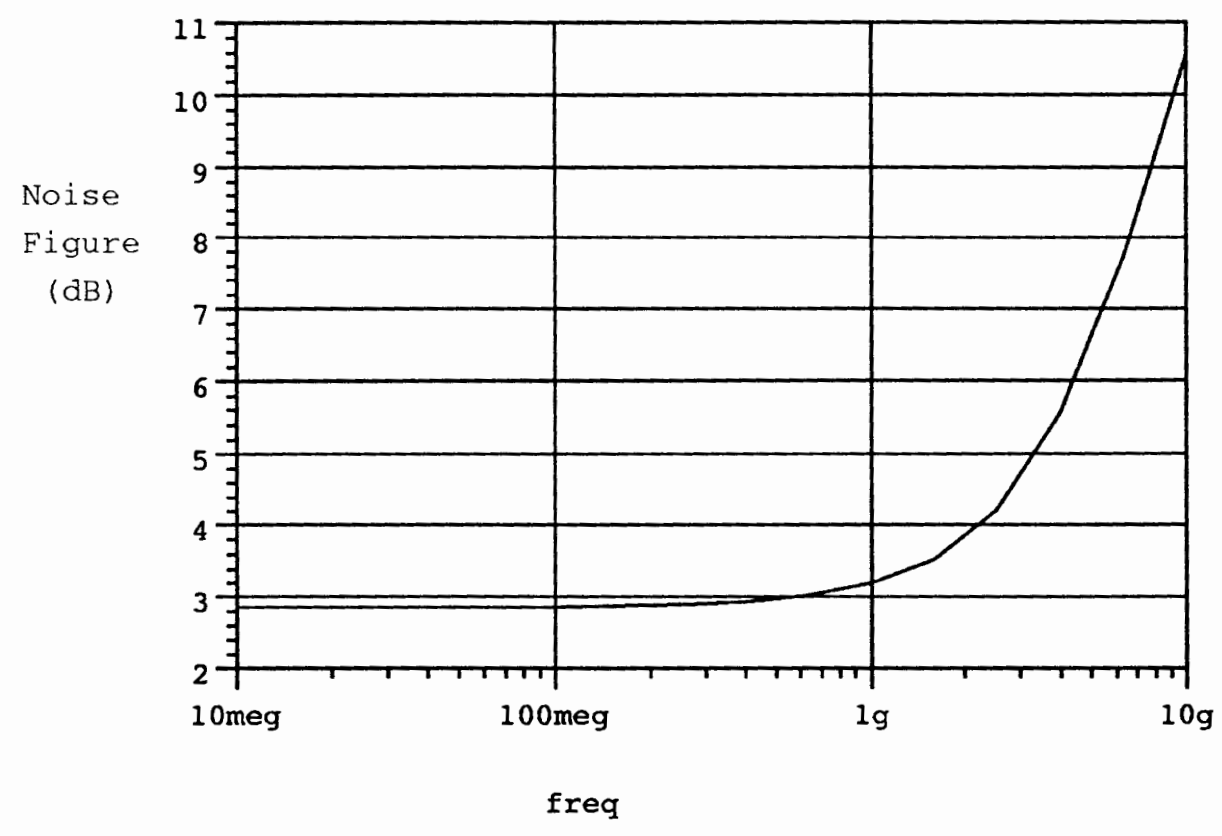

Figure 8. Noise figure vs. frequency for N16.

the noise figure of a N16 transistor with $V_{B E}=0.8 V, V_{C E}=4 V$, and $R_{s}=50 \Omega$ in common emitter configuration. 


\section{DETERMINATION OF NOISE PARAMETERS}

\section{Definition}

As defined in 1960 by the Institute of Radio Engineers Subcommittee on Noise [6], the noise figure depends upon the internal structure of the transducer and upon its input termination, but not upon its output termination. Thus, the noise performance of a transducer is meaningfully characterized by its noise figure only if the input termination is specified. The noise factor $F$ of any linear transducer, at a given operating point and input frequency, varies with the admittance $Y_{s}$ of its input termination in the following manner [7]:

$$
F=F_{o}+\frac{R_{n}}{G_{s}}\left|Y_{s}-Y_{o}\right|^{2}
$$

where $G_{s}$ is the real part of $Y_{s}$, and the parameters $F_{o}, Y_{o}$, and $R_{n}$ characterize the noise properties of the transducer and are independent of its input termination. Thus the noise performance of a transducer can be meaningfully characterized for all input terminations through specification of the parameters $F_{o}, Y_{o}$, and $R_{n}$. This source dependence of noise figure takes the form of the parabolic surface of Fig. 9.

The "optimum noise factor" $F_{o}$, at the given transducer operating point and frequency, is the lowest noise factor that can be obtained through adjustment of the source admittance $Y_{s}$. The "optimum source admittance" $Y_{o}$ is that particular value of source admittance $Y_{s}$ which results in optimum noise factor $F_{o}$. The parameter $R_{n}$ is positive and has the dimensions of a resistance. It is called the equivalent noise

resistance. This parameter appears as the coefficient of the $\left|Y_{s}-Y_{o}\right|^{2}$ term in the general expression for $F$ and, therefore, characterizes the rapidity with which $F$ increases above $F_{o}$ as $Y_{s}$ departs from $Y_{o}$. 


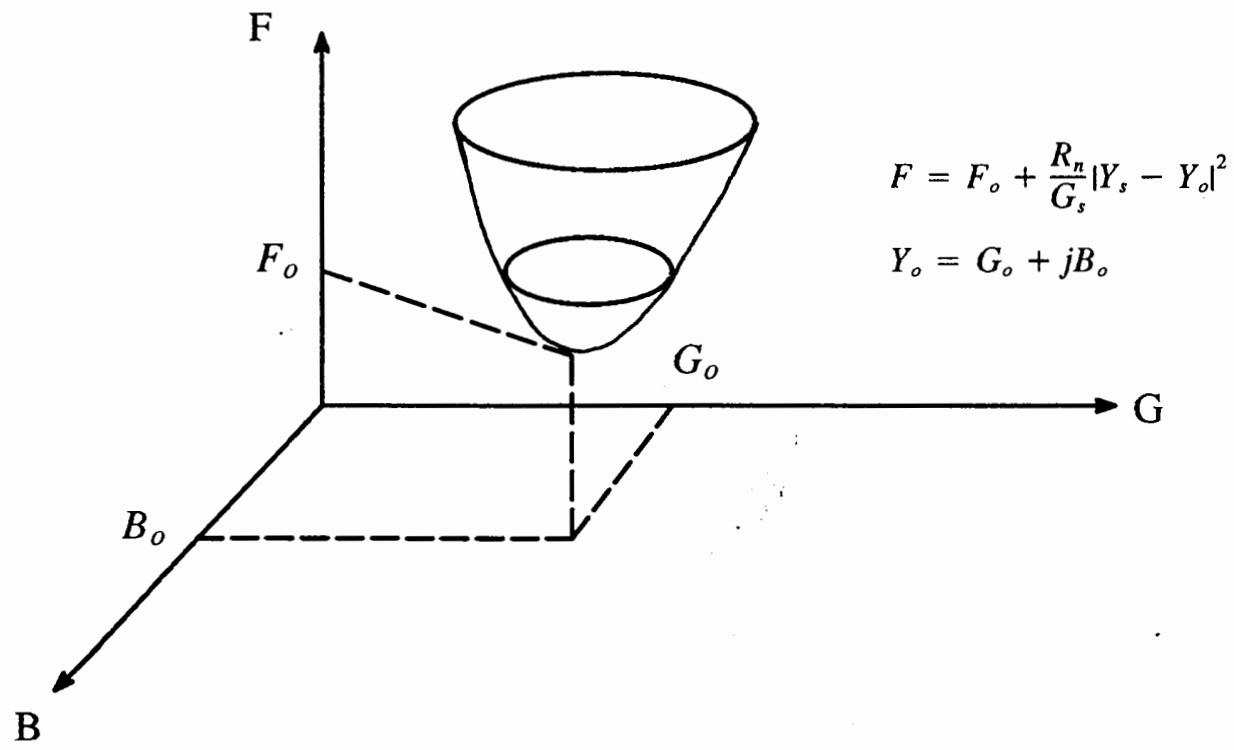

Figure 9. Parabolic noise surface.

\section{Source Impedance Selections}

The parameters $F_{o}, Y_{o}$, and $R_{n}$ can be calculated if the noise theory of the transducer is known or, alternatively, can be determine empirically from noise measurements.

Two methods of computer-aided determination of noise parameters have been reported in the literature.

One of them, Kokyczka et al [8], can be thought of as an automatic version of the graphic procedure suggested by the Institute of Radio Engineers [6], which required tedious and time-consuming adjustment of some input termination admittances with constant real part and of some other with constant imaginary part.

The other one, Lane [9], is an application of the least-squares method, which reduces the determination of noise parameters to the solution of a four linear equation system, obtained as fit of noise figures measured for different source admittances. In order to avoid singularity for the four linear equations, the proper selection of source 
admittances is essential for the accuracy of the noise parameters obtained. Garuso and Sannino [10] had suggested rules to choose source admittances to avoid singularity in the four linear equations. To completely define the parabolic surface given Eq. (25), the following conditions are to be AVOIDED in the selection of source admittance.

$$
\begin{aligned}
& G_{s}=\text { const } \\
& B_{s}=\text { const } \\
&\left|Y_{s}\right|^{2}=\text { const } \\
& \frac{\left|Y_{s}\right|^{2}}{G_{s}}=\text { const } \\
& \frac{\left|Y_{s}\right|^{2}}{B_{s}}=\text { const } \\
& \frac{\left|Y_{s}\right|^{2}}{G_{s}}=c+\frac{c^{\prime}}{G_{s}} \quad\left(c \text { and } c^{\prime} \text { are constants }\right)
\end{aligned}
$$

where $Y_{s}=G_{s}+j B_{s}$.

The reason for this will be explained in more detail under the topic "noise parameters computations".

\section{Noise Figure Measurement From TEKSPICE}

Noise figure is usually the way in which the noise performance of a component or system is quantified. A circuit designer requires device noise parameters to produce a circuit with specific noise figure performance. SPICE simulation can provide the needed noise figure data.

For noise figure testing in TEKSPICE, the schematic shown in Fig. 10 is used. 


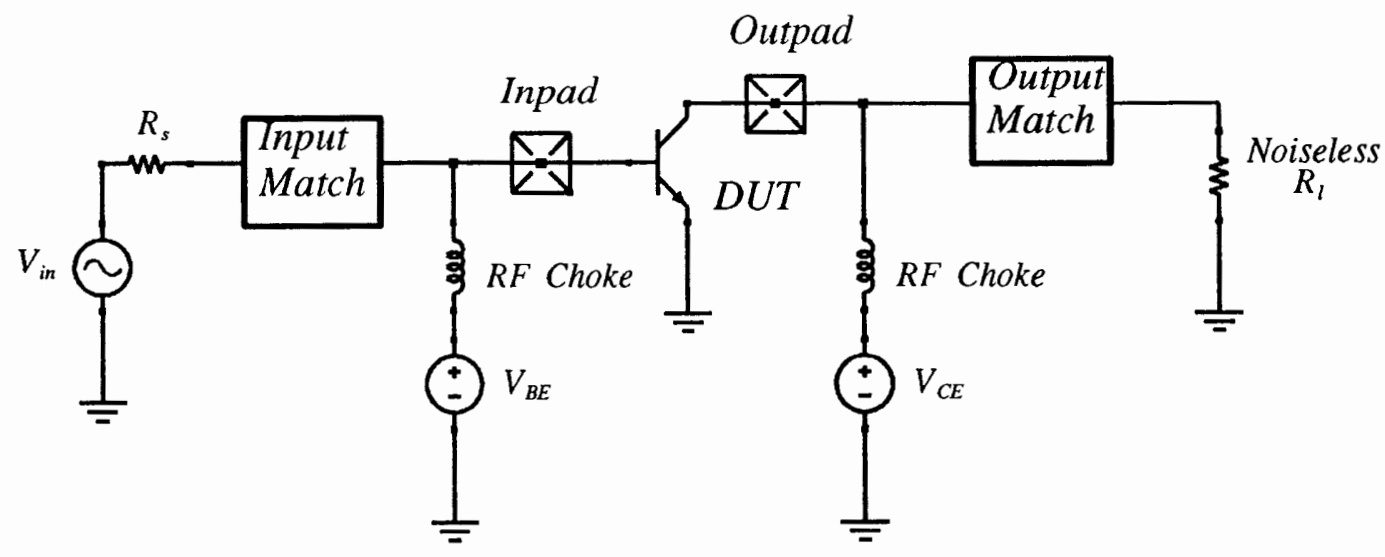

Figure 10. Test circuit for noise figure.

Noise figure is simulated by running a small-signal "AC analysis" with a "noise" statement included in the analysis block. Because SPICE linearizes the circuit in AC analysis, the input voltage source, $\mathrm{V}_{\mathrm{in}}$, is chosen to be $1 \mathrm{~V}$ to simplify calculations. The $\mathrm{AC}$ analysis calculates all the noise generated by the components in the circuit and refers it back to the input port. This noise is named as variable inoise. The algorithm to calculate noise figure in TEKSPICE is derived from the definition of noise figure.

$$
\begin{aligned}
F & =\frac{S_{i} / N_{i}}{S_{o} / N_{o}}=\frac{S_{i}}{S_{o}} \times \frac{N_{o}}{N_{i}}=\frac{1}{A_{v}} \times \frac{N_{o}}{N_{i}} \\
& =\frac{N_{o}}{A_{v}} \times \frac{1}{N_{i}} \\
N F & =10 \log _{10} F=10 \log _{10}\left(\frac{\text { inoise }^{2}}{4 K T R_{s}}\right)
\end{aligned}
$$

Since we are only interested in the noise generated by the transistor, we need to take out the noise generated by the load resistance. A "noiseless" resistive load is used. The noiseless resistor model is merely a "Current Controlled Voltage Source" (CCVS) with its transfer function set equal to $50 \Omega$, Fig. 11. A "noiseless" source resistance is not used because noise figure is defined as a ratio of signal-to-noise ratios, Eq. (34). A complete listing of the TEKSPICE script including the use of "noiseless" load is in Appendix B. 


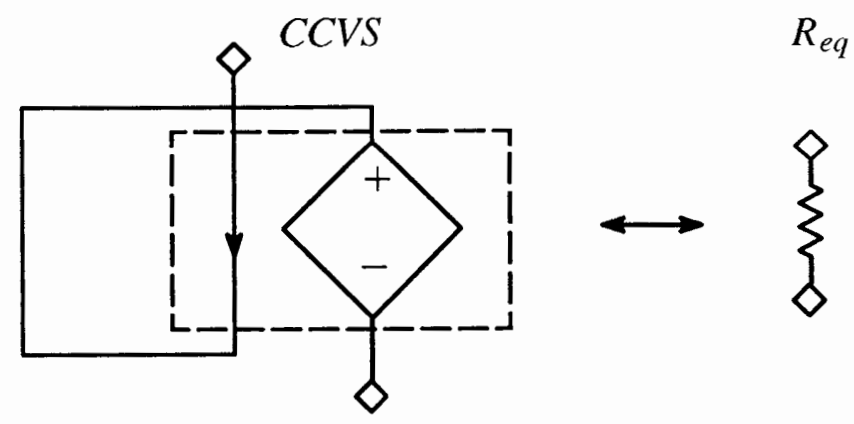

Figure 11. Noiseless resistor model.

Ten sets of data for the N16 and the G14V102 are obtained with different source impedance according to the criteria specified previously. They are tabulated in table I and will be used to calculate the noise parameters of the devices.

\section{TABLE I}

NOISE FIGURE MEASURED AT VARIOUS SOURCE IMPEDANCES WITH $V_{\mathrm{BE}}=0.8 \mathrm{~V}, \mathrm{~V}_{\mathrm{CE}}=4 \mathrm{~V}$ AT FREQUENCY $=1 \mathrm{GHZ}$

\begin{tabular}{|c|c|c|c|}
\hline \multirow{2}{*}{$\begin{array}{c}G_{s} \\
\text { mmhos }\end{array}$} & \multirow{2}{*}{$\begin{array}{c}B_{s} \\
\text { mmhos }\end{array}$} & \multicolumn{2}{|c|}{$\mathrm{d}$} \\
\cline { 3 - 4 } & & $\mathrm{N} 16$ & $\mathrm{G} 14 \mathrm{~V} 102$ \\
\hline \hline 6.33 & -2.26 & 2.258 & 2.724 \\
\hline 17.8 & 6.24 & 3.316 & 2.675 \\
\hline 24.9 & 6.64 & 3.783 & 2.830 \\
\hline 18.4 & 24.1 & 5.514 & 4.192 \\
\hline 7.14 & -7.14 & 2.702 & 2.697 \\
\hline 13.5 & -18.9 & 4.446 & 3.205 \\
\hline 44.9 & -28.1 & 5.751 & 3.948 \\
\hline 14.2 & -1.02 & 2.714 & 2.365 \\
\hline 15.7 & -3.92 & 2.861 & 2.362 \\
\hline 22.8 & -7.12 & 3.509 & 2.585 \\
\hline
\end{tabular}




\section{Noise Parameters Computation}

After the noise figure data for different source impedances are obtained, the minimum noise figure, the optimum source resistance, and the equivalent noise resistance can be determined. Eq. (25) is rewritten to be the following to eliminate the magnitude sign:

$$
F=F_{o}+\frac{R_{n}}{G_{s}}\left[\left(G_{s}-G_{o}\right)+j\left(B_{s}-B_{o}\right)\right]^{2}
$$

In order to use a readily available subroutine for solving simultaneous equation solution, Eq. (35) is transformed to a form that is linear with respect to four new parameters $A, B$, $C$, and $D[11]$.

$$
F=A+B G_{s}+\frac{C+B B_{s}^{2}+D B_{s}}{G_{s}}
$$

where

$$
\begin{gathered}
F_{o}=A+\sqrt{4 B C-D^{2}} \\
R_{n}=B \\
G_{o}=\frac{\sqrt{4 B C-D^{2}}}{2 B} \\
B_{o}=\frac{-D}{2 B} .
\end{gathered}
$$

In principle, four measurements of noise factor from different source admittances will determine the four real numbers ( $F_{o}, R_{n}, G_{o}$, and $B_{o}$ ). Eq. (35) becomes overdetermined if more than four measurements are taken, but by minimizing the square of error as expressed in Eq. (41), more than four measurements can be used to find those parameters which give the best least squares fit to Eq. (35). It has been shown that only slight variations of noise parameters occur versus redundancy if the number of data sets processed is greater than 7 [10]. A least-squares fit of the ten simulated noise figures from table I to the plane of Eq. (36) is sought: therefore, the following error criterion is 
established [9]:

$$
\varepsilon=\frac{1}{2} \sum_{i=1}^{n} W_{i}\left[A+B\left(G_{i}+\frac{B_{i}^{2}}{G_{i}}\right)+\frac{C}{G_{i}}+\frac{D B_{i}}{G_{i}}-F_{i}\right]^{2}
$$

where $W_{i}$ is the weighting factor to be used if certain data are known to be less reliable than the average. In this simulation, each measurement has the same reliability, therefore $\sum_{i=1}^{10} W_{i}=1$. Then:

$$
\begin{gathered}
\frac{\delta \varepsilon}{\delta A}=\sum_{i=1}^{10} W_{i} P=\sum_{i=1}^{10} P=0 \\
\frac{\delta \varepsilon}{\delta B}=\sum_{i=1}^{10} W_{i}\left(G_{i}+\frac{B_{i}^{2}}{G_{i}}\right) P=\sum_{i=1}^{10}\left(G_{i}+\frac{B_{i}^{2}}{G_{i}}\right) P=0 \\
\frac{\delta \varepsilon}{\delta C}=\sum_{i=1}^{10} W_{i} \frac{P}{G_{i}}=\sum_{i=1}^{10} \frac{P}{G_{i}}=0 \\
\frac{\delta \varepsilon}{\delta D}=\sum_{i=1}^{10} W_{i} \frac{B_{i}}{G_{i}} P=\sum_{i=1}^{10} \frac{B_{i}}{G_{i}} P=0
\end{gathered}
$$

where

$$
P=\left[A+B\left(G_{i}+\frac{B_{i}^{2}}{G_{i}}\right)+\frac{C}{G_{i}}+\frac{D B_{i}}{G_{i}}-F_{i}\right]
$$

The coefficient matrix of the four linear equations is established from Eq. (42) through Eq. (45): 


$$
\left[\begin{array}{llll}
10 & \sum_{i=1}^{10}\left(G_{i}+\frac{B_{i}^{2}}{G_{i}}\right) & \sum_{i=1}^{10} \frac{1}{G_{i}} & \sum_{i=1}^{10} \frac{B_{i}}{G_{i}} \\
\sum_{i=1}^{10}\left(G_{i}+\frac{B_{i}^{2}}{G_{i}}\right) & \sum_{i=1}^{10}\left(G_{i}+\frac{B_{i}^{2}}{G_{i}}\right)^{2} & \sum_{i=1}^{10}\left(1+\frac{B_{i}^{2}}{G_{i}^{2}}\right) & \sum_{i=1}^{10} B_{i}+\frac{B_{i}^{3}}{G_{i}^{2}} \\
\sum_{i=1}^{10} \frac{1}{G_{i}} & \sum_{i=1}^{10}\left(1+\frac{B_{i}^{2}}{G_{i}^{2}}\right) & \sum_{i=1}^{10} \frac{1}{G_{i}^{2}} & \sum_{i=1}^{10} \frac{B_{i}}{G_{i}^{2}} \\
\sum_{i=1}^{10} \frac{B_{i}}{G_{i}} & \sum_{i=1}^{10} B_{i}+\frac{B_{i}^{3}}{G_{i}^{2}} & \sum_{i=1}^{10} \frac{B_{i}}{G_{i}^{2}} & \sum_{i=1}^{10}\left(\frac{B_{i}}{G_{i}}\right)^{2}
\end{array}\right]
$$

By inspection of this matrix, some values of the source admittance $Y_{s}$ can be recognized which can cause the matrix to become singular. Some of these values are the one for which a column (row) is proportional to another one or can be obtained as a linear combination of the other ones. The values of such source admittance are listed earlier in Eq. (26) to Eq. (31).

A Pascal program is written to process the noise data. The matrix Eq. (47) is solved by using a subroutine available from "Numerical Recipes Example Book" [12]. A complete description and a listing of the entire program are included in Appendix C. The N16 and G14V102 are simulated under the same conditions for comparison. These data are then processed by the developed Pascal program. Table II shows the noise parameters for the N16 and G14V102 transistors with $V_{B E}=0.8 \mathrm{~V}, V_{C E}=4 \mathrm{~V}$ at $f=1 G H z$. 
TABLE II

NOISE PARAMETERS OF N16 AND G14V102

TRANSISTORS

\begin{tabular}{|c|c|c|c|c|}
\hline & $\begin{array}{c}F_{o} \\
\mathrm{~dB}\end{array}$ & $\begin{array}{c}R n \\
\text { ohms }\end{array}$ & $\begin{array}{c}G o \\
\text { mmhos }\end{array}$ & $\begin{array}{c}\text { Bo } \\
\text { mmhos }\end{array}$ \\
\hline \hline $\mathrm{N} 16$ & 2.25 & 42.67 & 6.31 & -1.66 \\
\hline G14V102 & 2.34 & 21.32 & 13.12 & -3.43 \\
\hline
\end{tabular}

\section{NOISE FIGURE CIRCLES}

As stated previously, the noise figure of a two port network is given by

$$
F=F_{o}+\frac{R_{n}}{G_{s}}\left|Y_{s}-Y_{o}\right|^{2}
$$

To plot noise figures on a Smith chart, $Y_{s}$ and $Y_{o}$ are expressed in terms of reflection coefficients $\Gamma_{s}$ and $\Gamma_{o}$, which are:

$$
Y_{s}=\frac{1-\Gamma_{s}}{1+\Gamma_{s}}
$$

and

$$
Y_{o}=\frac{1-\Gamma_{o}}{1+\Gamma_{o}}
$$

Substituting Eq. (49) and Eq. (50) into Eq. (48) results in the following relation:

$$
F=F_{o}+\frac{4 R_{n}\left|\Gamma_{s}-\Gamma_{o}\right|^{2}}{\left(1-\left|\Gamma_{s}\right|^{2}\right)\left|1+\Gamma_{o}\right|^{2}}
$$

This equation can be used to design $\Gamma_{s}$ for a given noise figure. To determine a family of noise figure circles, an intermediate noise figure parameter, $N_{i}$, is defined [1]. 


$$
\begin{aligned}
N_{i} & =\frac{\left|\Gamma_{s}-\Gamma_{o}\right|^{2}}{1-\left|\Gamma_{s}\right|^{2}} \\
& =\frac{F_{i}-F_{o}}{4 R_{n} / Z_{o}}\left|1+\Gamma_{o}\right|^{2}
\end{aligned}
$$

Eq. (52) is then transformed to:

$$
\left|\Gamma_{s}-\frac{\Gamma_{o}}{1+N_{i}}\right|^{2}=\frac{N_{i}^{2}+N_{i}\left(1-\left|\Gamma_{o}\right|^{2}\right)}{\left(1+N_{i}\right)^{2}}
$$

Eq. (54) is recognized as a family of circles with $N_{i}$ as a parameter. The center and radius of the circle can be found from:

$$
C_{F_{i}}=\frac{\Gamma_{o}}{1+N_{i}}
$$

and

$$
R_{F_{i}}=\frac{1}{1+N_{i}} \sqrt{N_{i}^{2}+N_{i}\left(1-\left|\Gamma_{o}\right|^{2}\right)}
$$

Eq. (53), Eq. (55), and Eq. (56) show that when $F_{i}=F_{o}$, then $N_{i}=0, C_{F_{o}}=\Gamma_{o}$, and $R_{F_{o}}=0$. That is, the center of the $F_{o}$ circle is located at $\Gamma_{o}$ with zero radius. From Eq. (55), the centers of the other noise figure circles are located along the $\Gamma_{o}$ vector.

The developed Pascal program, which is also used to calculate noise parameters, calculates the constant noise figure circles (with an increment of $0.5 \mathrm{~dB}$ from the minimum noise figure). A set of constant noise figure circles for the N16 is shown in Fig. 12. This plot gives information about the noise figure of the device for different source impedances at $1 \mathrm{GHz} . \mathrm{A} F_{i} d B$ noise figure circle on the plot specifies the values of source impedance at which the device will produce a noise figure of $F_{i}$. This set of curves show that the minimum noise figure, $F_{o}=2.25 \mathrm{~dB}$ is obtained when $\Gamma_{s}=\Gamma_{o}=0.52 \angle 10.6^{\circ}$, and at point A, $\Gamma_{s}=0.31 \angle 80^{\circ}$ produces $F_{i}=3.25 \mathrm{~dB}$. 
One point of interest is on the center of the Smith chart, which corresponds to a source impedance of $50 \Omega$. The noise figure at this point is what we can have if the device is put into a $50 \Omega$ environment without any tuning circuitry at the input ports. 


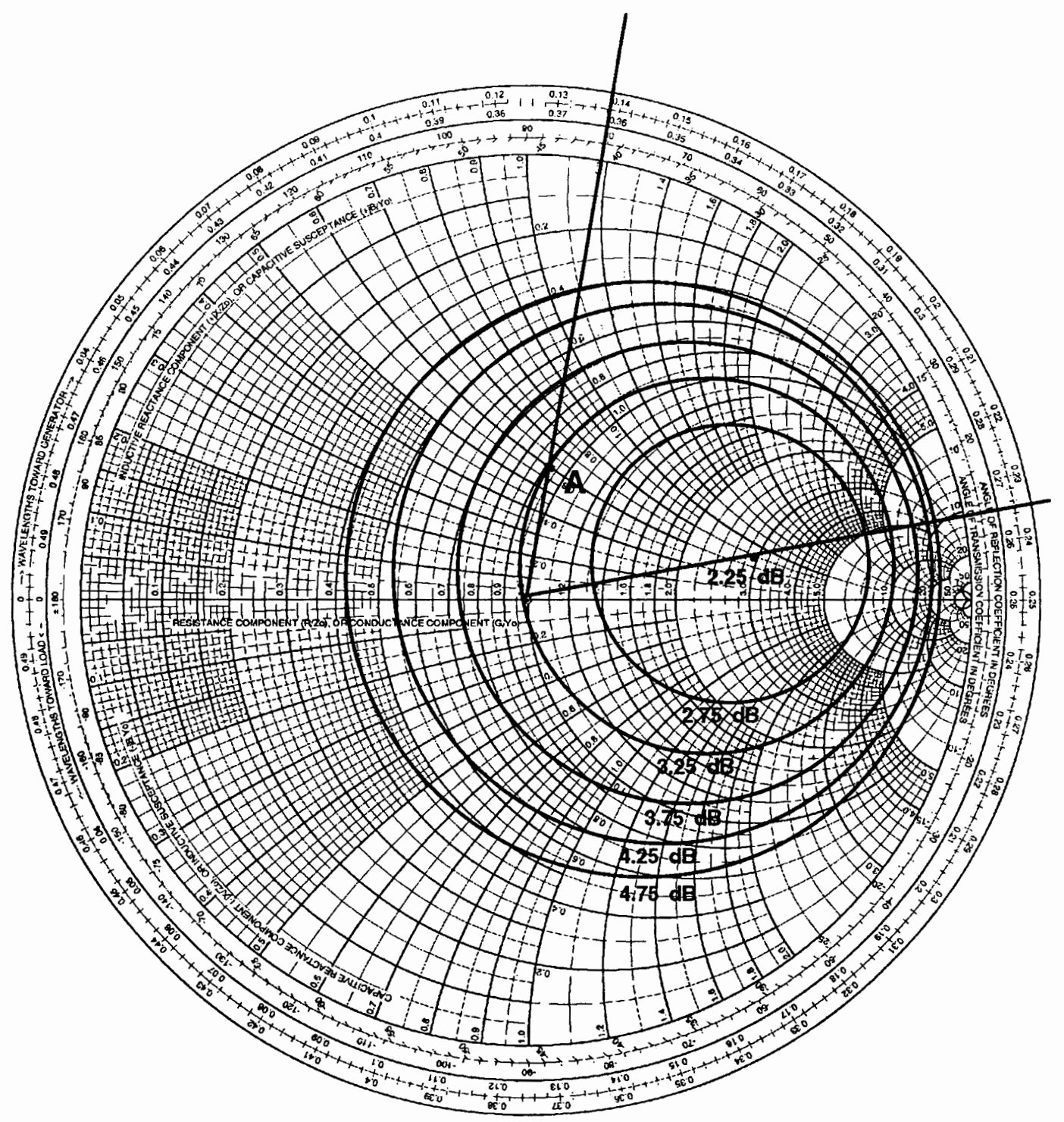

Figure 12. Constant noise figure circles for $\mathrm{N} 16$ with $\mathrm{V}_{\mathrm{BE}}=0.8 \mathrm{~V}$, $\mathrm{V}_{\mathrm{CE}}=4 \mathrm{~V}$ at Frequency $=1 \mathrm{GHz}$. 


\section{COMPARISON}

From the data obtained, the equivalent noise resistance $\left(R_{n}\right)$ of the G14V102 is smaller than the one of the N16. This is verified by wider spacing of noise figure circles of the G14V102 as compare to the ones of the N16 (Fig. 13 vs. Fig. 14, Fig. 15 vs. Fig. 16). The lower of $R_{n}$ will result in reduced sensitivity of the noise figure to changes in source impedance. Therefore, a circuit designer can have more freedom on choosing source impedances for better power gain and/or better input matching for a given noise figure. 


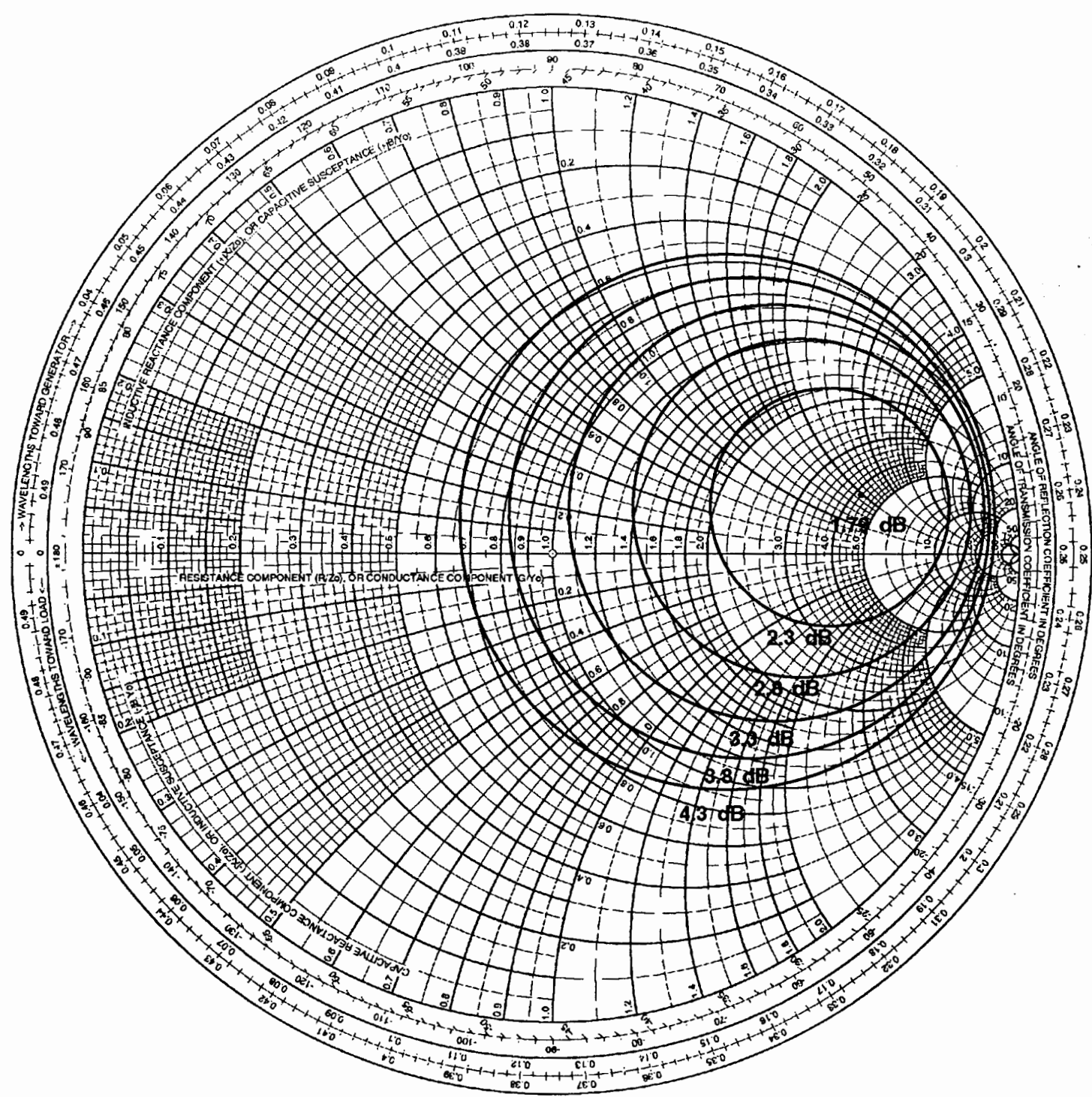

Figure 13. Constant noise figure circles for $\mathrm{N} 16$ with $\mathrm{V}_{\mathrm{BE}}=0.76 \mathrm{~V}$, $\mathrm{V}_{\mathrm{CE}}=4 \mathrm{~V}$ at frequency $=900 \mathrm{MHz}$. 


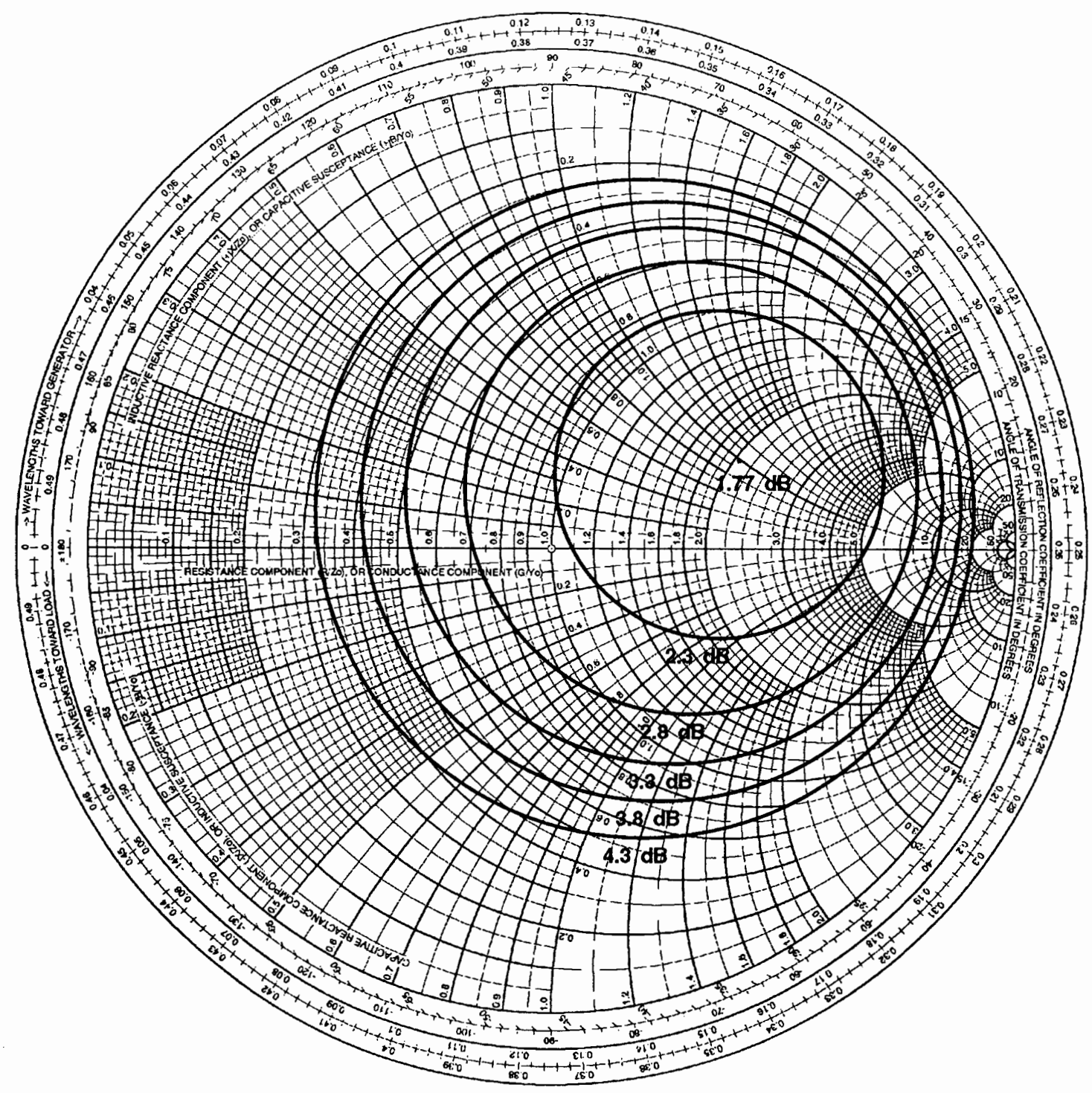

Figure 14. Constant noise figure circles for G14V102 with $\mathrm{V}_{\mathrm{BE}}=0.76 \mathrm{~V}$, $\mathrm{V}_{\mathrm{CE}}=4 \mathrm{~V}$ at frequency $=900 \mathrm{MHz}$. 


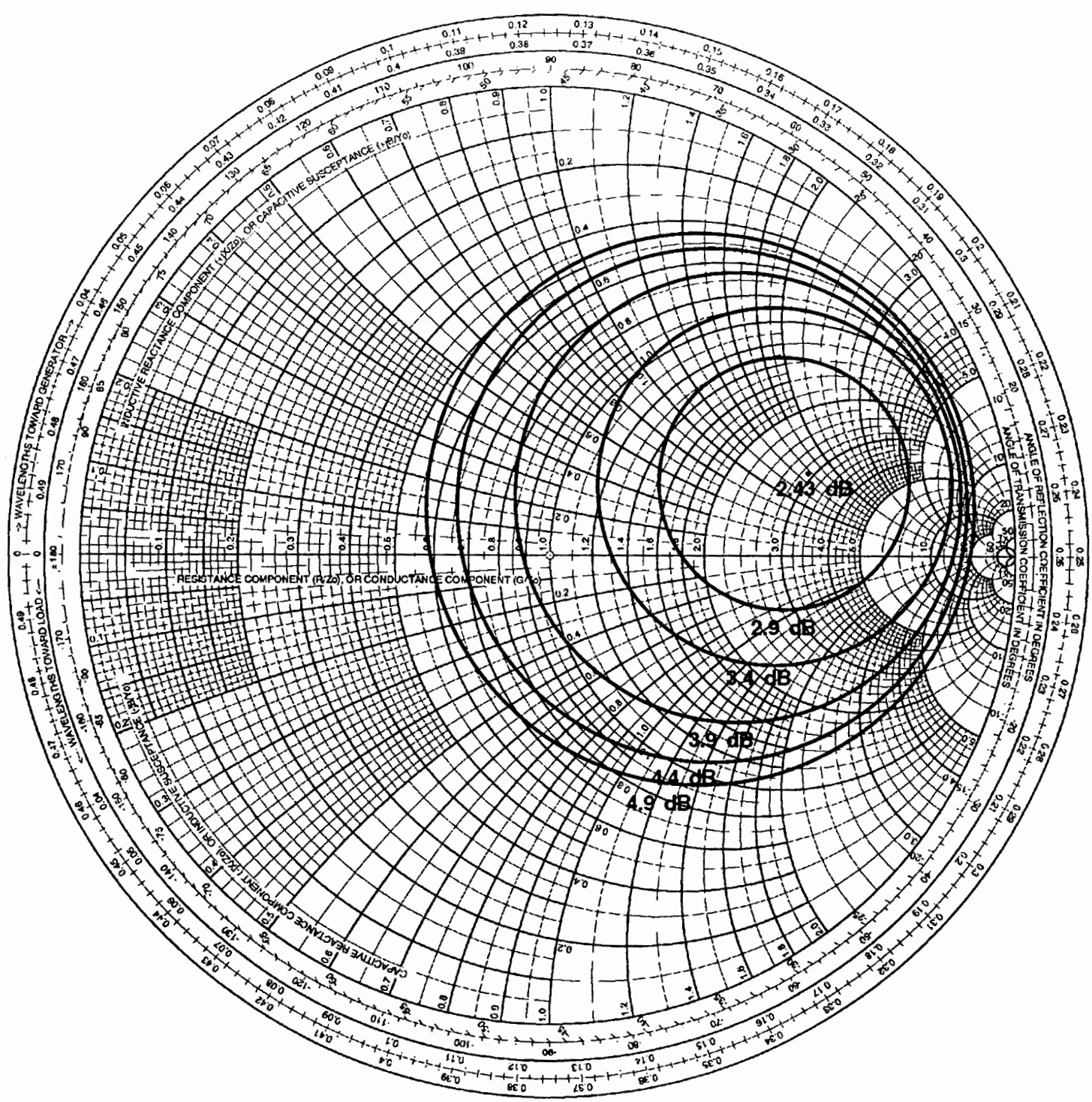

Figure 15. Constant noise figure circles for N16 with $\mathrm{V}_{\mathrm{BE}}=0.76$, $\mathrm{V}_{\mathrm{CE}}=4 \mathrm{~V}$ at frequency $=1.6 \mathrm{GHz}$. 


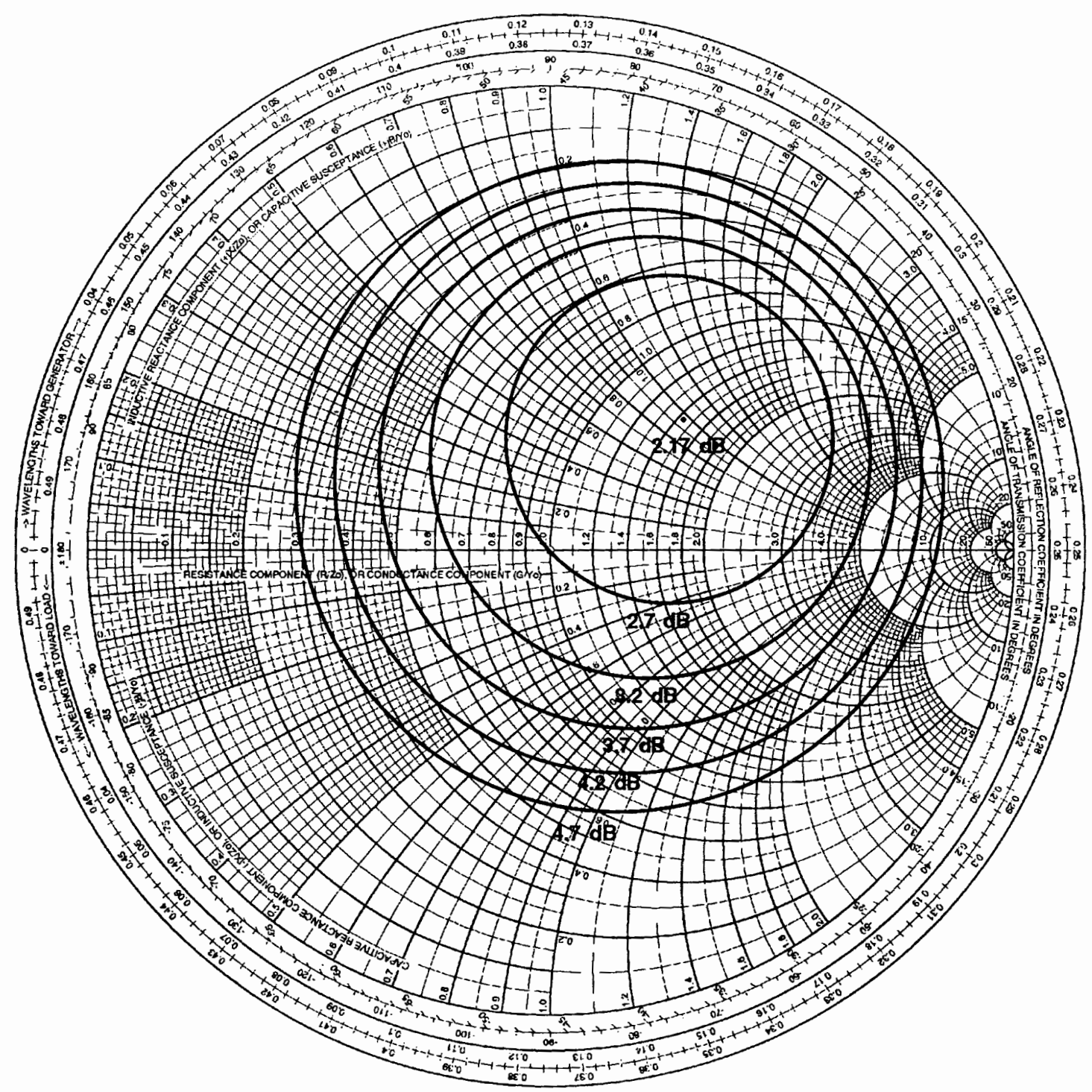

Figure 16. Constant noise figure circles for G14V102 with $\mathrm{V}_{\mathrm{BE}}=0.76 \mathrm{~V}, \mathrm{~V}_{\mathrm{CE}}=4 \mathrm{~V}$ at Frequency $=1.6 \mathrm{GHz}$. 


\section{CHAPTER IV}

\section{GAIN MEASUREMENT}

In this chapter, the gain and stability of the bipolar transistors in terms of s-parameters will be investigated. A procedure for extracting s-parameters from SPICE is presented. Finally, a comparison between the simulated and measured s-parameter data will be made.

\section{GAIN AND STABILITY}

\section{Stability Criteria}

As the operating frequency of the transistor is being pushed upward, the transistor is more prone to unwanted oscillation due to parasitic elements. From feedback theory, we know that the input resistance of the common emitter circuit shown in Fig. 17 is

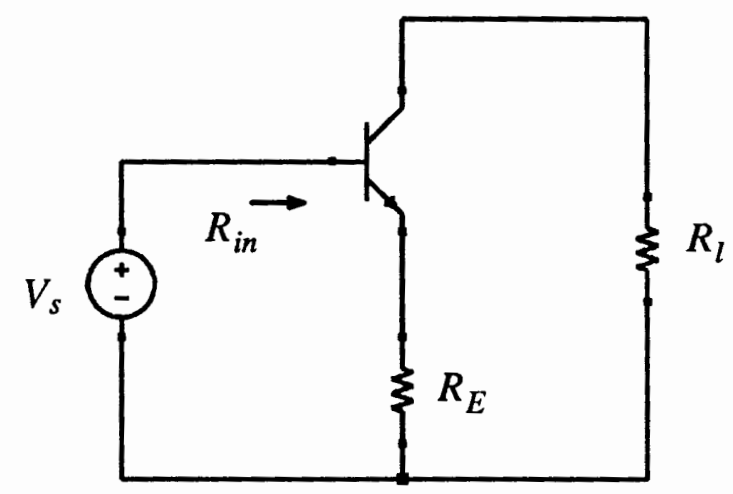

Figure 17. Common emitter amplifier with local series feedback.

$R_{\text {in }}=R_{\text {in }}{ }^{\prime}+(1+\beta) R_{E}$, where $R_{\text {in }}{ }^{\prime}$ is the input resistance of the circuit without the emitter degeneration resistor $R_{E}$. There is a multiplying factor of $(1+\beta)$ for $R_{E}$. This 
is obvious because the base current is $\frac{1}{1+\beta}$ times the emitter current. The resistive parasitic in the emitter, which can be treated the same way as $R_{E}$ in Fig. 17, is being reflected to the base by the complex $\beta$ as an inductive component at high frequencies. This inductive reactance and the parasitic capacitance in the transistor may cause the transistor to oscillate at high frequencies.

The necessary conditions for stability of a two-port device like bipolar transistors, had been studied by Kurokawa[13], Bodway[14], and Woods[15]. In terms of s-parameters, they are:

$$
|\Delta|=\left|S_{11} S_{22}-S_{12} S_{21}\right|<1
$$

and

$$
K=\frac{1-\left|S_{11}\right|^{2}-\left|S_{22}\right|^{2}+|\Delta|^{2}}{2\left|S_{12} S_{21}\right|}>1
$$

Two-port devices that meets the above criteria are unconditionally stable for any positive source and load impedance.

\section{Stability Circles}

To maximize the gain, we must conjugately match the input and the output. For unconditionally stable two-ports networks there is no unwanted oscillation to worry about. But for those networks which cannot meet the above stability criteria, we will have to look at what might happen to the network in terms of stability--will the amplifier oscillate with certain values of impedance used in the matching process?

In a two-port network, oscillations are possible when either the input or output port presents a negative resistance, since noise generated in the adjoining network enters the port, the negative resistance generates more noise rather than dissipating the incident noise, and some of this generated noise combines with the incoming noise to input more noise. Negative resistances correspond to the points outside the Smith chart, which 
imply either $\left|\Gamma_{\text {in }}\right|>1$ or $\left|\Gamma_{\text {out }}\right|>1$. Therefore, we have the boundary for the input and output stability circles defined:

$$
\left|\Gamma_{\text {in }}\right|=\left|S_{11}+\frac{S_{12} S_{21} \Gamma_{l}}{1-S_{22} \Gamma_{l}}\right|=1
$$

and

$$
\left|\Gamma_{\text {out }}\right|=\left|S_{22}+\frac{S_{12} S_{21} \Gamma_{s}}{1-S_{11} \Gamma_{s}}\right|=1
$$

Solving for the values of $\Gamma_{l}$ and $\Gamma_{s}$ in Eq. (59) and Eq. (60) shows that the solutions for $\Gamma_{l}$ and $\Gamma_{s}$ lie on circles [2].

The radius and center of the input stability circles are:

$$
\begin{aligned}
& r_{s}=\left|\frac{S_{12} S_{21}}{\left|S_{11}\right|^{2}-|\Delta|^{2}}\right| \\
& C_{s}=\frac{\left(S_{11}-\Delta S_{22}^{*}\right)^{*}}{\left|S_{11}\right|^{2}-|\Delta|^{2}}
\end{aligned}
$$

and the radius and center of the output stability circles are:

$$
\begin{aligned}
& r_{l}=\left|\frac{S_{12} S_{21}}{\left|S_{22}\right|^{2}-|\Delta|^{2}}\right| \\
& C_{l}=\frac{\left(S_{22}-\Delta S_{11}^{*}\right)^{*}}{\left|S_{22}\right|^{2}-|\Delta|^{2}}
\end{aligned}
$$

where

$$
\Delta=S_{11} S_{22}-S_{12} S_{21}
$$

Having measured the s-parameters of a two-port device at one frequency, Eq. (61) to Eq. (64) can be evaluated, and plotted on a Smith chart. Fig. 18 illustrates the graphical construction of the stability circles where $\left|\Gamma_{\text {in }}\right|=1$. On one side of the stability circles boundary, in the $\Gamma_{l}$ plane, we will have $\left|\Gamma_{\text {in }}\right|<1$ and on the other side $\left|\Gamma_{\text {in }}\right|>1$. 


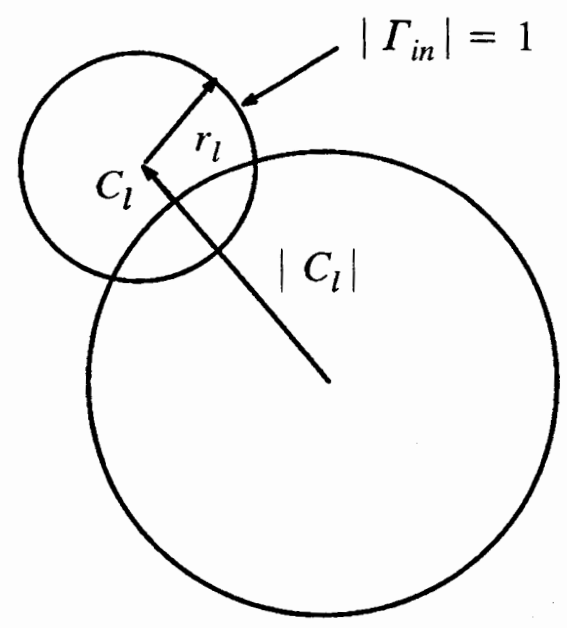

Figure 18. Stability circles construction on a Smith chart.

To determine which area represents the stable operating condition, we make $\Gamma_{l}=0$, which is to terminate the output port with a $50 \Omega$ load through a $50 \Omega$ transmission line. This represents the point at the center of the Smith chart. Under these conditions,

$$
\left|\Gamma_{i n}\right|=\left|S_{11}\right|
$$

For N16, the magnitude of $S_{11}$ measured is less than unity, therefore, the center of the Smith chart represents a stable operating point. That is, the shade area on Fig. 19 represents $\left|\Gamma_{\text {in }}\right|<1$. The same procedure applies for finding the output stability region.

When the input and output stability circles lie completely outside the Smith chart the network is called unconditionally stable for all $\Gamma_{s}$ and $\Gamma_{l}$. This comes from the fact that no matter what positive termination is put at the input or output of the network $\left|\Gamma_{\text {in }}\right|$ and $\left|\Gamma_{\text {out }}\right|$ will be always less than unity.

The TEKSPICE script for calculating input and output stability circles based on Eq. (61) to Eq. (65) is listed in Appendix D. 


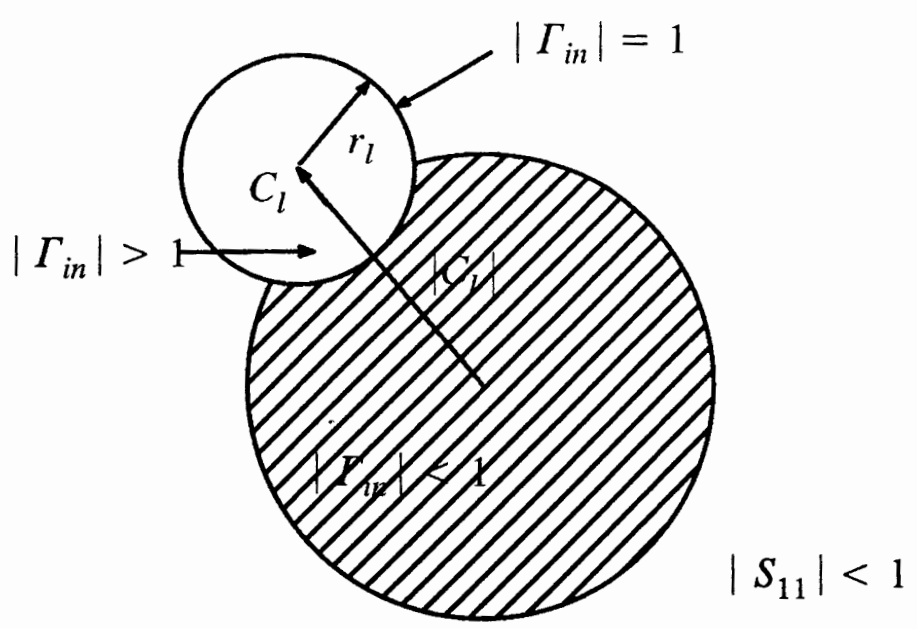

Figure 19. Stability region for $\Gamma_{\mathrm{s}}$.

\section{Gain Circles}

S-parameters can be used to predict the available power gain of a transistor for any input termination $\Gamma_{s}$. This available gain is that gain achieved when a transistor is driven from some source reflection $\Gamma_{s}$ while terminated with a load impedance equal to $\Gamma_{\text {out }}^{*}$ (matched output). The available power gain in terms of reflection coefficients is [1]:

$$
G_{A}=\frac{\left(1-\left|\Gamma_{s}\right|^{2}\right)}{\left|1-S_{11} \Gamma_{s}\right|^{2}}\left|S_{21}\right|^{2} \frac{1}{\left(1-\left|\Gamma_{\text {out }}\right|^{2}\right)}
$$

where

$$
\Gamma_{\text {out }}=S_{22}+\frac{S_{12} S_{21} \Gamma_{s}}{1-S_{11} \Gamma_{s}}
$$

Because $G_{A}$ is a function of the source reflection coefficient, constant available power gain circles can be plotted on a Smith chart together with the constant noise figure circles, and the trade-off result between gain and noise figure can be analyzed.

For a given gain $G_{A}$, the radius $R_{a}$ and the center $C_{a}$ of the circle can be calculated using the relations [2] . 


$$
\begin{gathered}
g_{a}=\frac{G_{A}}{\left|S_{21}\right|^{2}} \\
C_{1}=S_{11}-\Delta S_{22}^{*} \\
R_{a}=\frac{\sqrt{1-2 K\left|S_{12} S_{21}\right| g_{a}+\left|S_{12} S_{21}\right|^{2} g_{a}^{2}}}{\left|1+g_{a}\left(\left|S_{11}\right|^{2}-|\Delta|^{2}\right)\right|}
\end{gathered}
$$

and

$$
C_{a}=\frac{g_{a} C_{1}^{*}}{1+g_{a}\left(\left|S_{11}\right|^{2}-|\Delta|^{2}\right)}
$$

For a given $G_{A}$, the constant available power gain circle can be plotted. All $\Gamma_{s}$ on this circle produce the gain $G_{A}$. A TEKSPICE script for calculating the radius and center of gain circles is given in Appendix $\mathrm{E}$.

\section{S-PARAMETERS FROM TEKSPICE}

From the previous discussion, gain and stability circles of a two-port network can be obtained from manipulation of s-parameters of the network. In addition to noise figure data, SPICE simulation can provide s-parameters of a two-port network as well. S-parameters are ratios of incident and reflected wave at the ports of a network. In order to get s-parameters from TEKSPICE simulation, they need to be calculated in terms of input and output voltages and currents [16]. For the two-port network shown in Fig. 20, the input reflection coefficient can be calculated from node voltages:

$$
\begin{aligned}
S_{11} & =\frac{V_{1}-Z_{s} I_{1}}{V_{1}+Z_{s} I_{1}} \\
& =\frac{V_{1}-\left(V_{s}-V_{1}\right)}{V_{1}+\left(V_{s}-V_{1}\right)} \\
& =\frac{2 V_{1}-V_{s}}{V_{s}}
\end{aligned}
$$

where $V_{1}, V_{s}$ and $S_{11}$ are complex variables. 


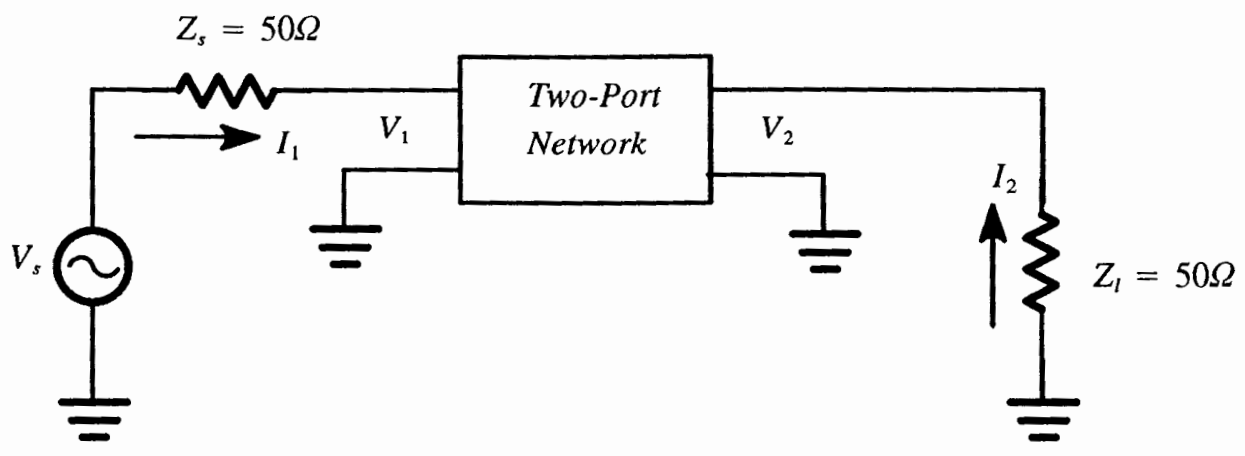

Figure 20. Test circuit for obtaining $S_{11}$ and $S_{21}$.

If $V_{s}=2 V \angle 0^{\circ}$, then Eq. (75) is simplified to:

$$
S_{11}=V_{1}-1
$$

For the forward transmission coefficient, the deviation is followed:

$$
\begin{aligned}
S_{21} & =\frac{V_{2}-Z_{l} I_{2}}{V_{1}+Z_{s} I_{1}} \\
& =\frac{V_{2}-\left(-V_{2}\right)}{V_{1}+\left(V_{s}-V_{1}\right)} \\
& =\frac{2 V_{2}}{V_{s}}
\end{aligned}
$$

where $V_{2}, V_{s}$ and $S_{21}$ are also complex variables.

If $V_{s}=2 V \angle 0^{\circ}$, then $S_{21}$ is just simply:

$$
S_{21}=V_{2}
$$

For $S_{22}$ and $S_{12}$, the same technique is employed. This time the network is reversed, as shown in Fig. 21. 


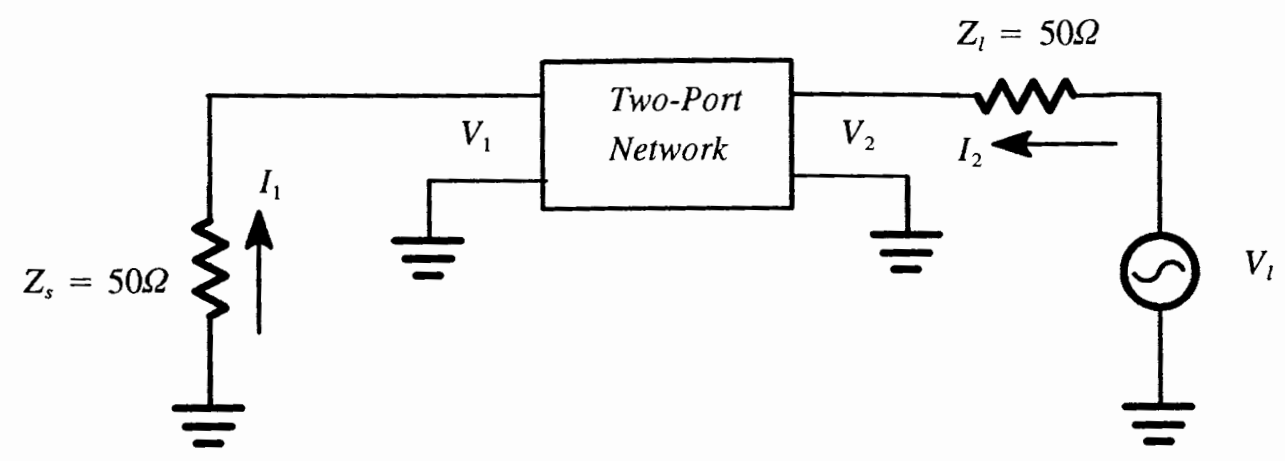

Figure 21. Test circuit for obtaining $S_{22}$ and $S_{12}$.

With $V_{l}=2 V \angle 0^{\circ}$, they are:

$$
\begin{aligned}
& S_{22}=V_{2}-1 \\
& S_{12}=V_{1}
\end{aligned}
$$

A TEKSPICE script for performing the above arithmetic is listed in detail in Appendix F.

\section{COMPARISON}

For an N16 with $V_{B E}=0.76 \mathrm{~V}, V_{C E}=4 \mathrm{~V}$ and $f=900 \mathrm{MHz}$, the measured and simulated s-parameter data are plotted in Fig. 22 to Fig. 29. The simulated data are based on the device models provided by Tektronix. Those models have been proven to be representing the actual devices closely by many successful ICs manufactured by Tektronix. Some deviations between the simulated data and the measured data can be noticed, (Fig. 23, Fig. 25, and Fig. 29). These are all happening in the phase of the measured s-parameters. This is traced to a problem with the network analyzer and its frequency generator. The frequency from the generator is not locked onto the network analyzer. Even though the frequencies are the same, they are not necessarily in phase. When the frequencies are matched, measured and simulated data agree with each other, (Fig. 27). The magnitudes of the measured s-parameters (Fig. 22, Fig. 24, Fig. 26, and Fig. 28) are in agreement with the simulated data. This proves the method employed to 
obtain s-parameters from SPICE is valid. To avoid this frequency mismatch problem, a frequency synthesizer under the control of the network analyzer needs to replace the frequency sweep generator currently used.

Gain and stability circles for the N16 and the G14V102 are plotted in Fig. 30 and Fig. 31. Under the conditions $V_{B E}=0.76 \mathrm{~V}, V_{C E}=4 \mathrm{~V}$ at $f=900 \mathrm{MHz}$, these two transistors can provide similar power gains.

Finally, the gain and noise figure circles are plotted in Fig. 32 and Fig. 33. From Fig. 32, the N16 will provide a gain of $16 \mathrm{~dB}$ and a noise figure of $3.5 \mathrm{~dB}$ with $50 \Omega$ source impedance under the conditions $V_{B E}=0.76 \mathrm{~V}, V_{C E}=4 \mathrm{~V}$ at $f=900 \mathrm{MHz}$, while a G14V102 will provide about the same gain but with a superior noise figure of $2.4 \mathrm{~dB}$ under the same condition, Fig. 33.

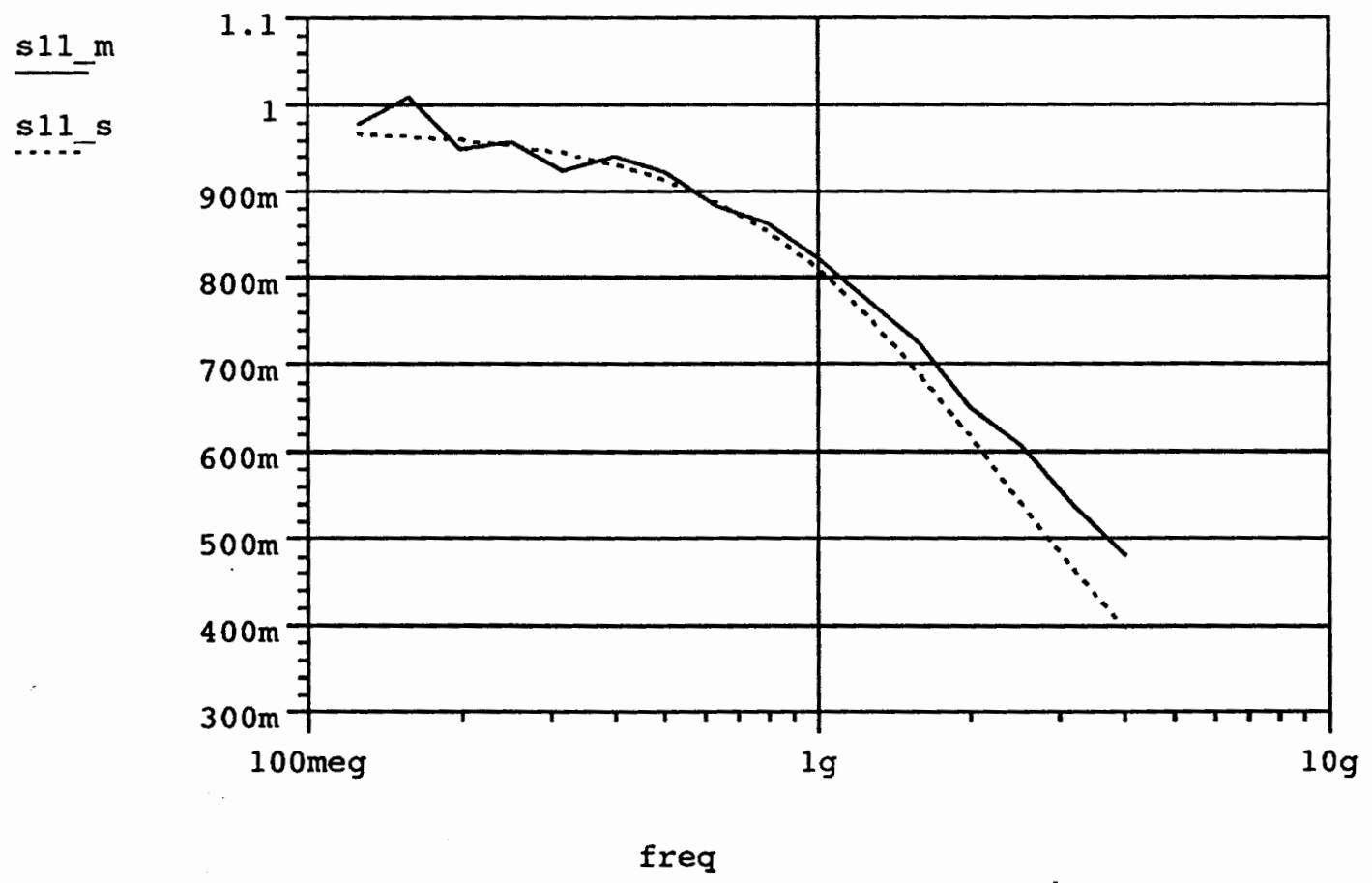

Figure 22. Magnitude of $S_{11}$ for N16. 


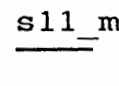

s11_s

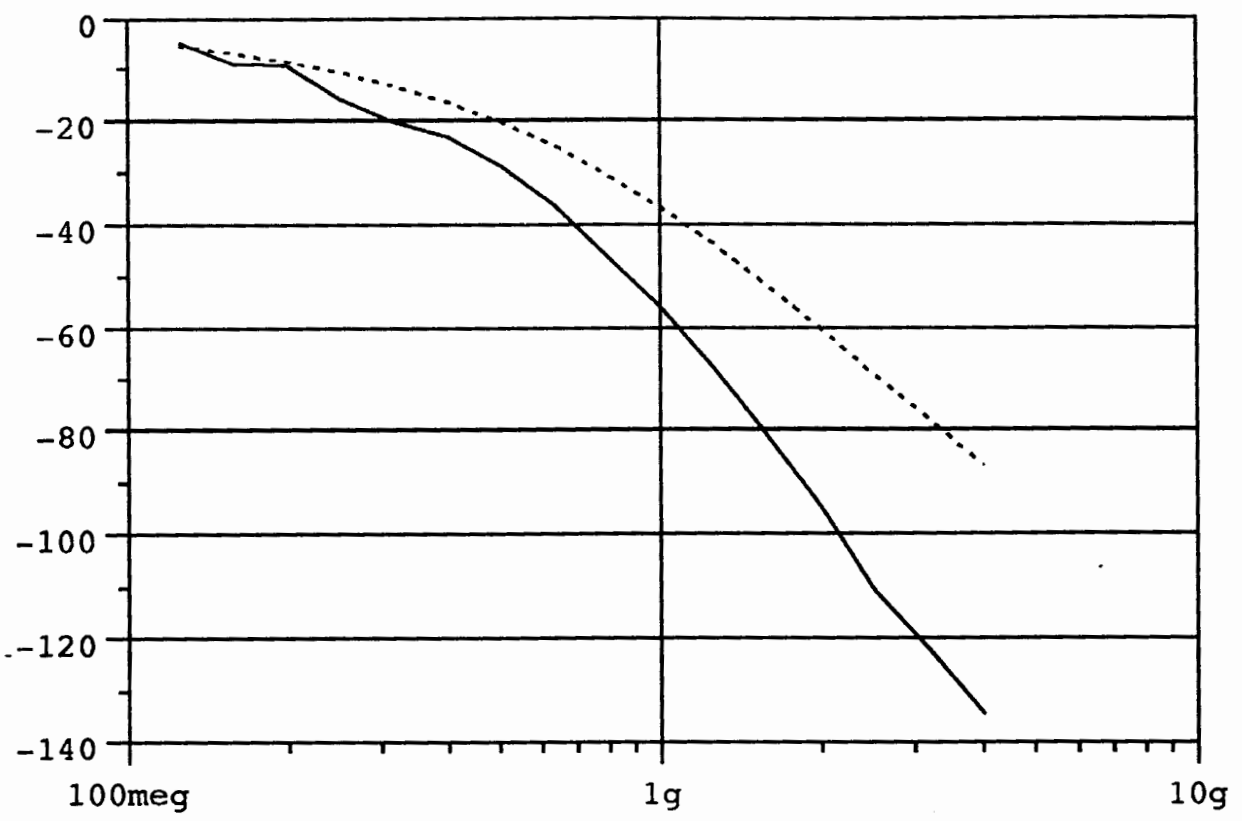

freq

Figure 23. Phase of $S_{11}$ for N16.

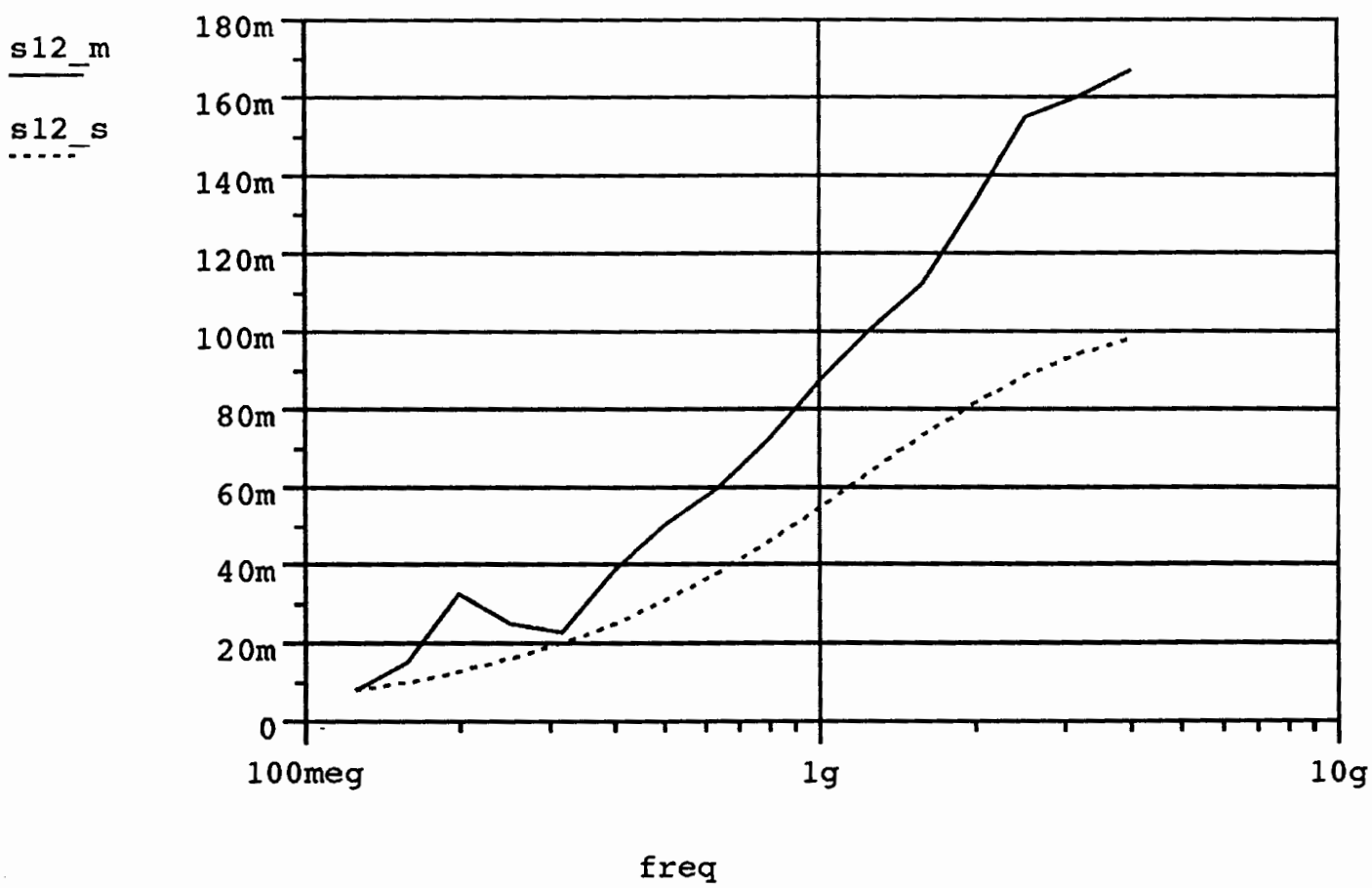

Figure 24. Magnitude of $S_{12}$ for N16. 
$\mathrm{s} 12 \mathrm{~m}$

s 12 s

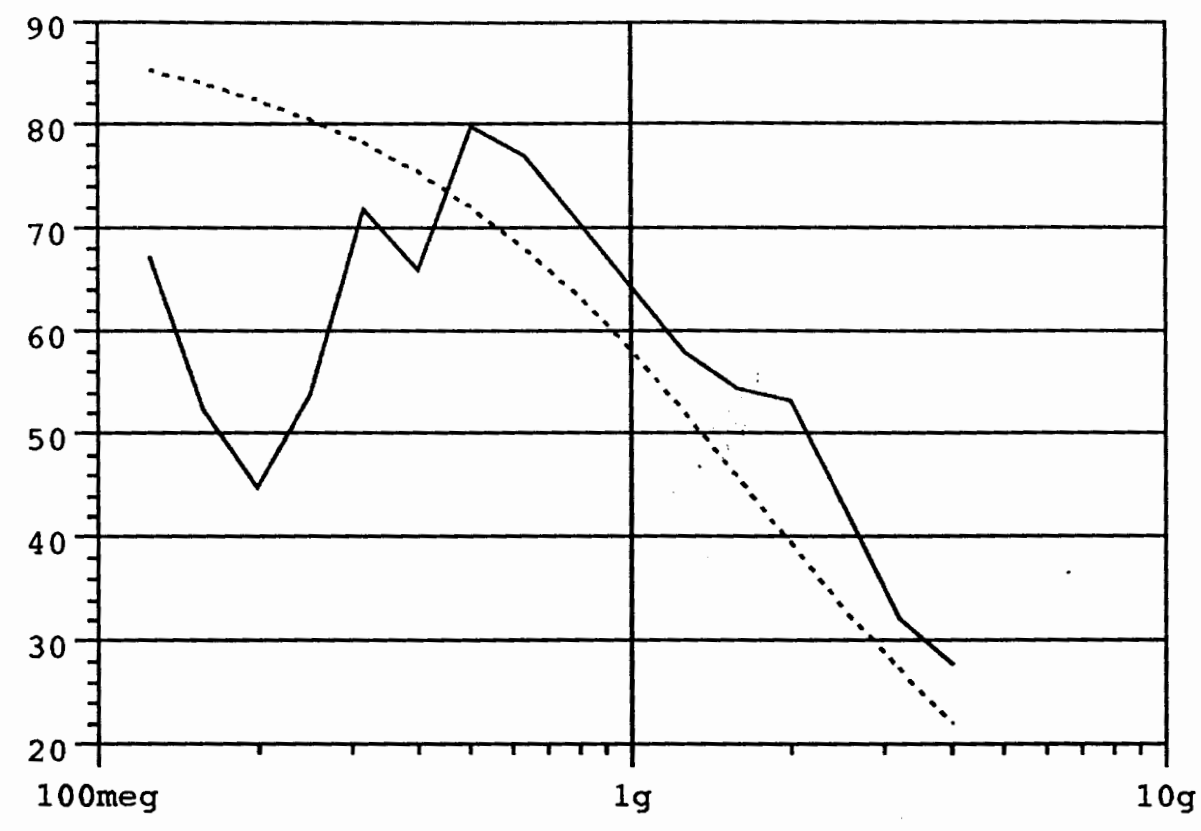

freq

Figure 25. Phase of $S_{12}$ for N16.

s21_m
s21_s

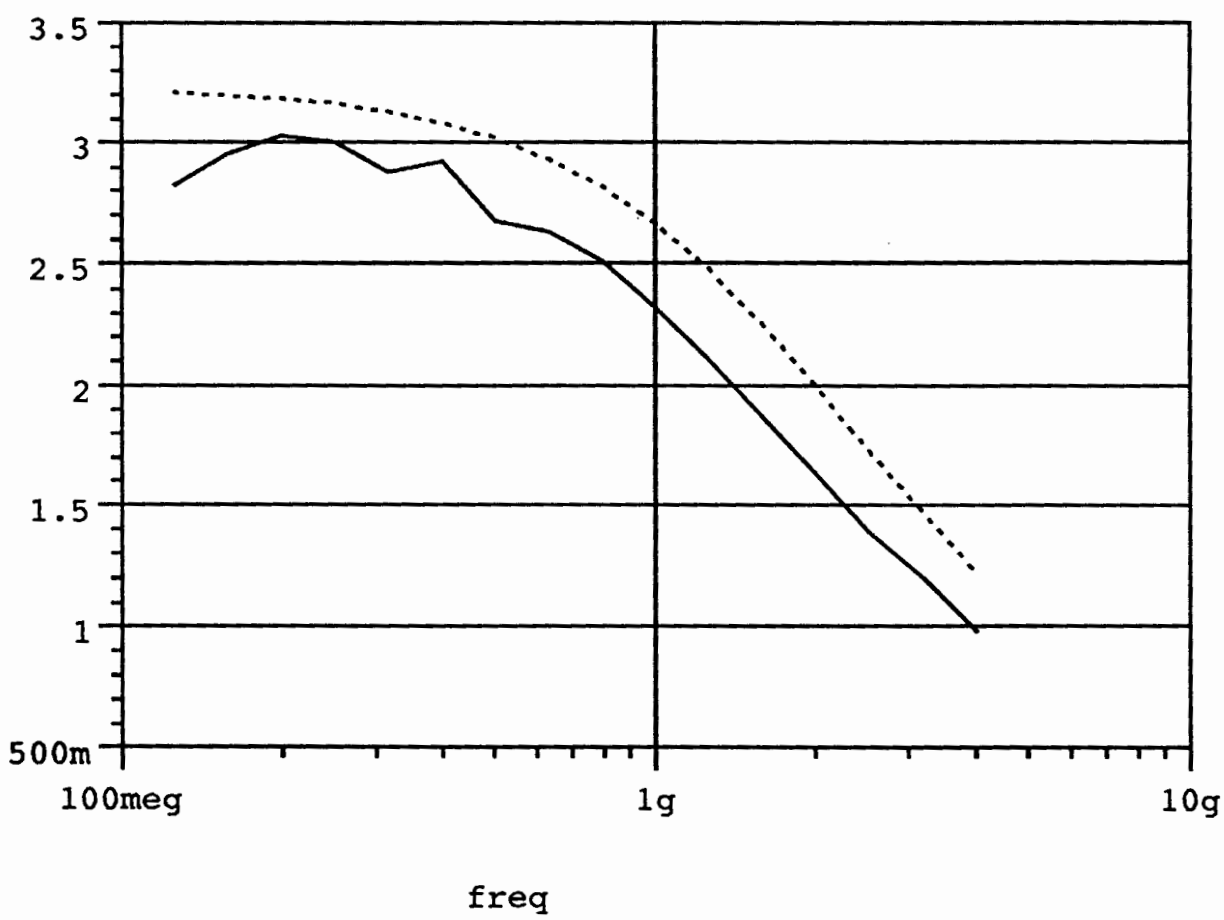

Figure 26. Magnitude of $S_{21}$ for N16. 


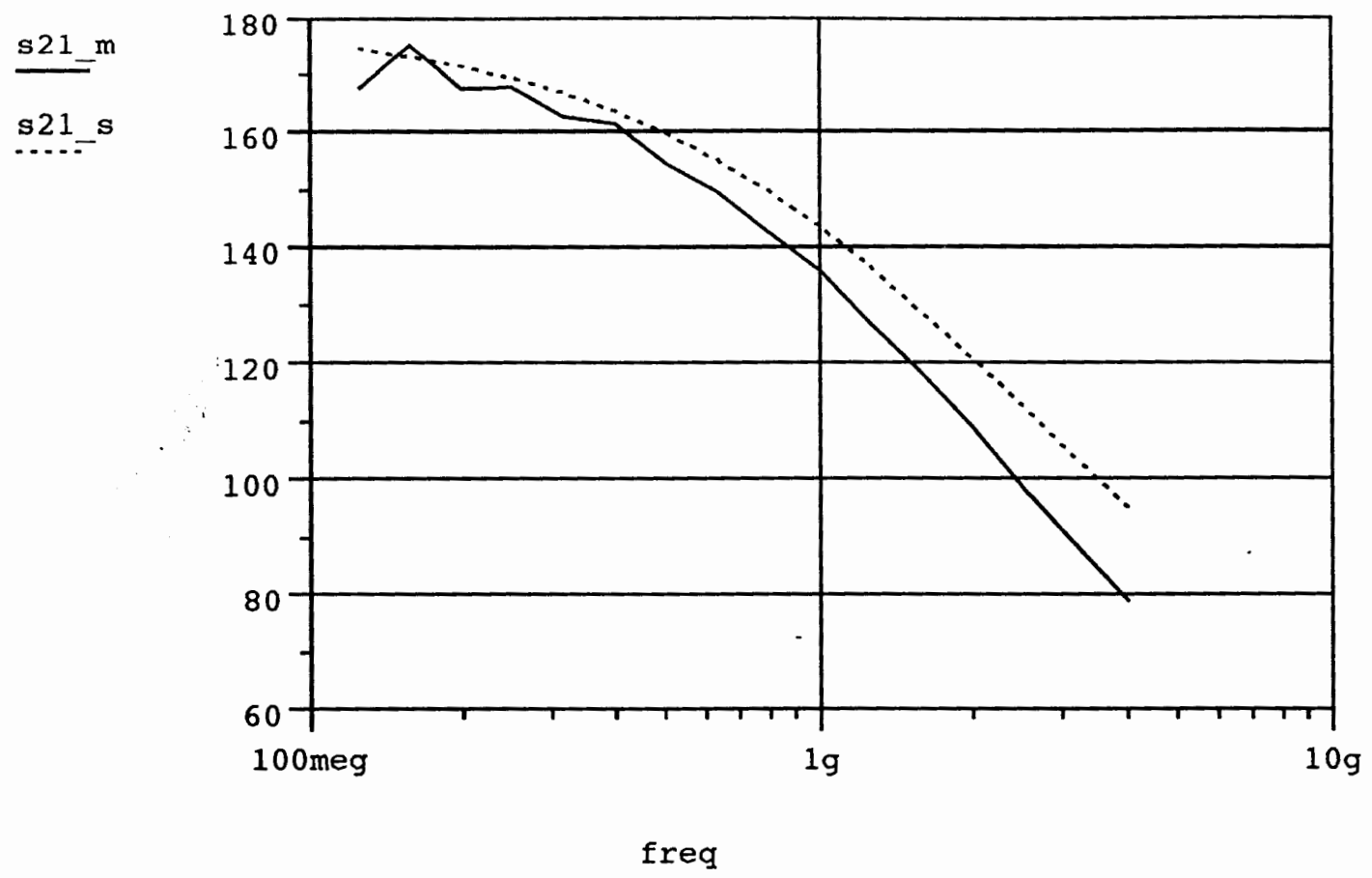

Figure 27. Phase of $S_{21}$ for N16.
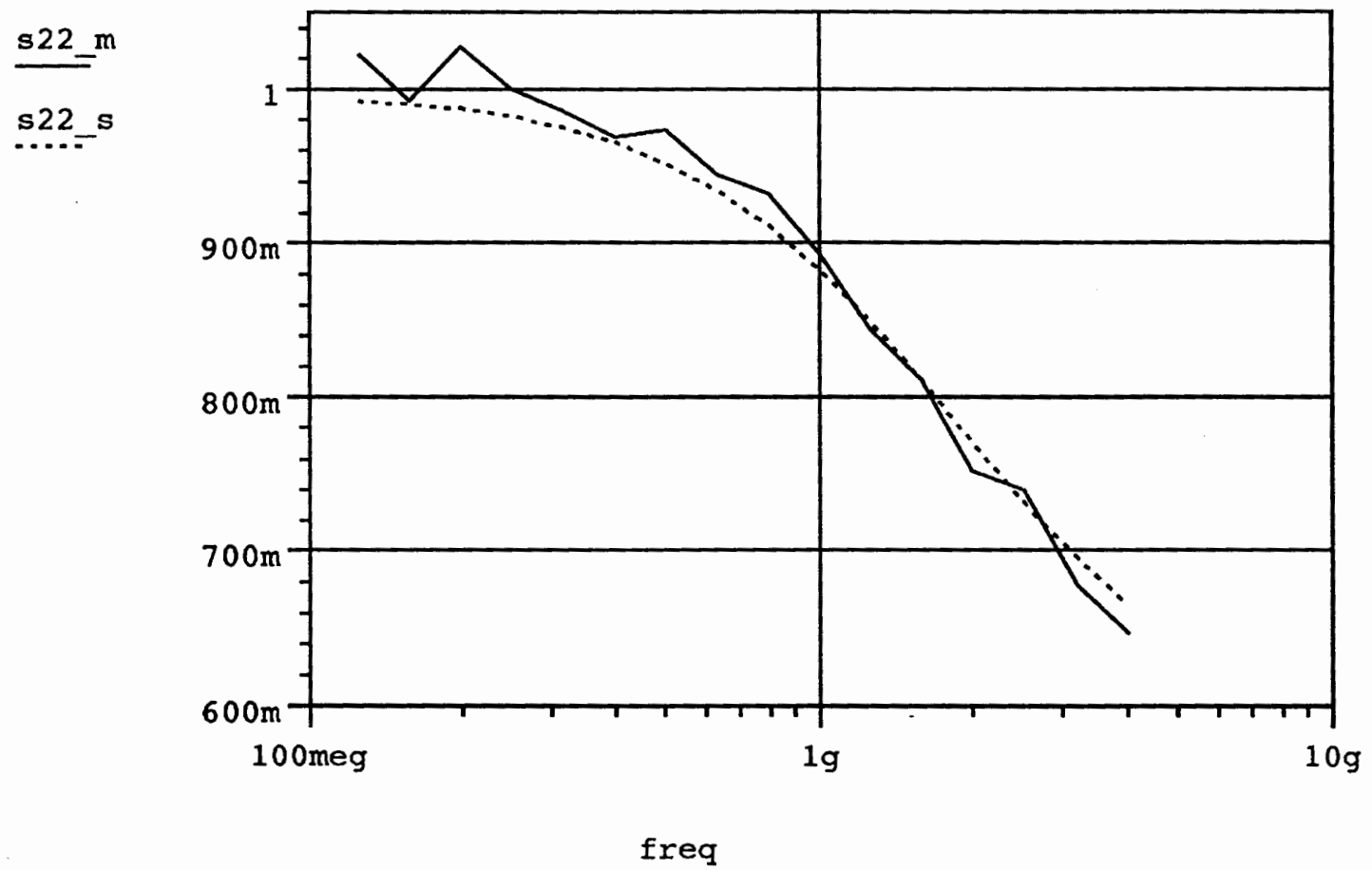

Figure 28. Magnitude of $\mathrm{S}_{22}$ for N16. 

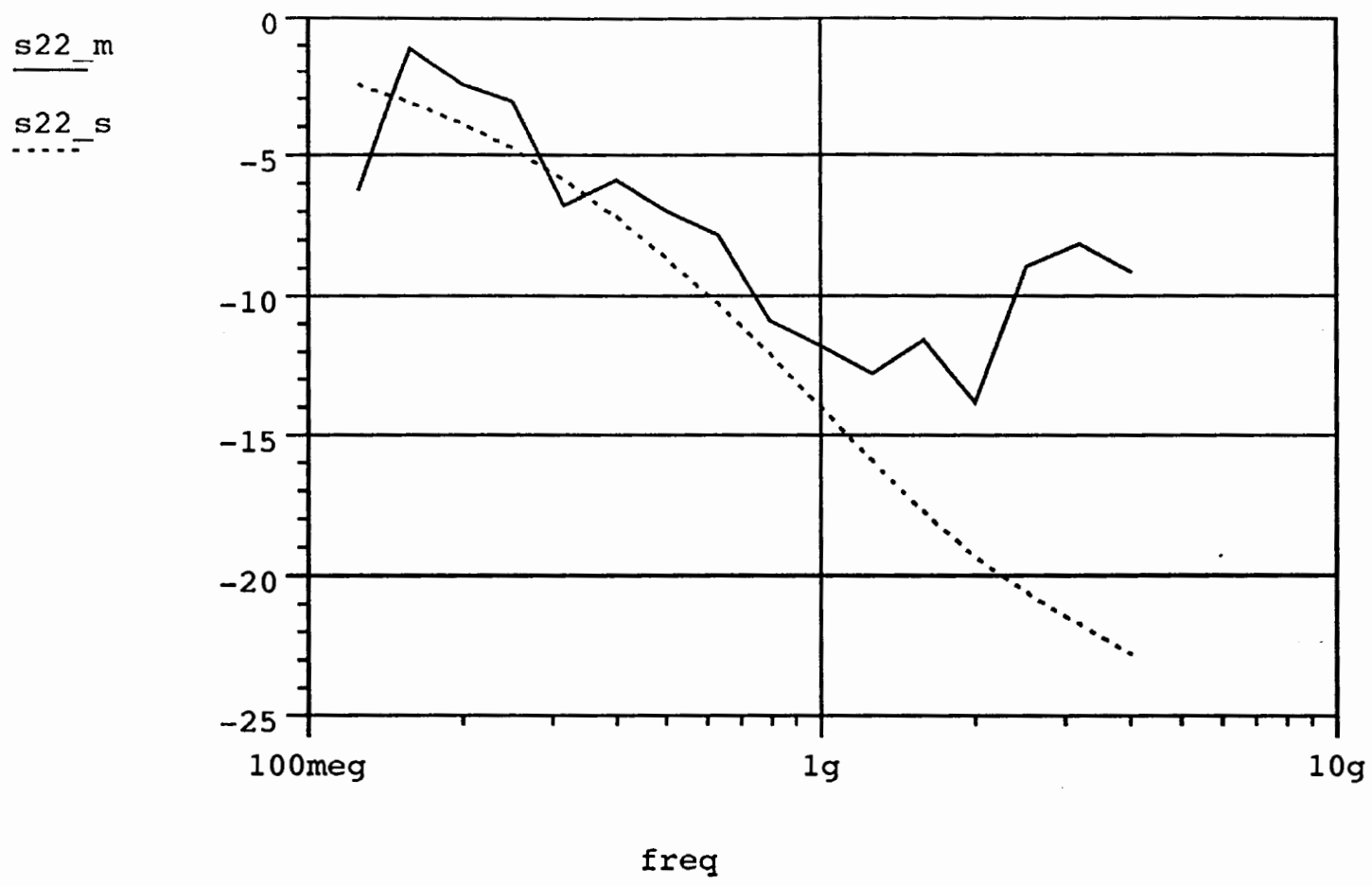

Figure 29. Phase of $S_{22}$ for N16. 


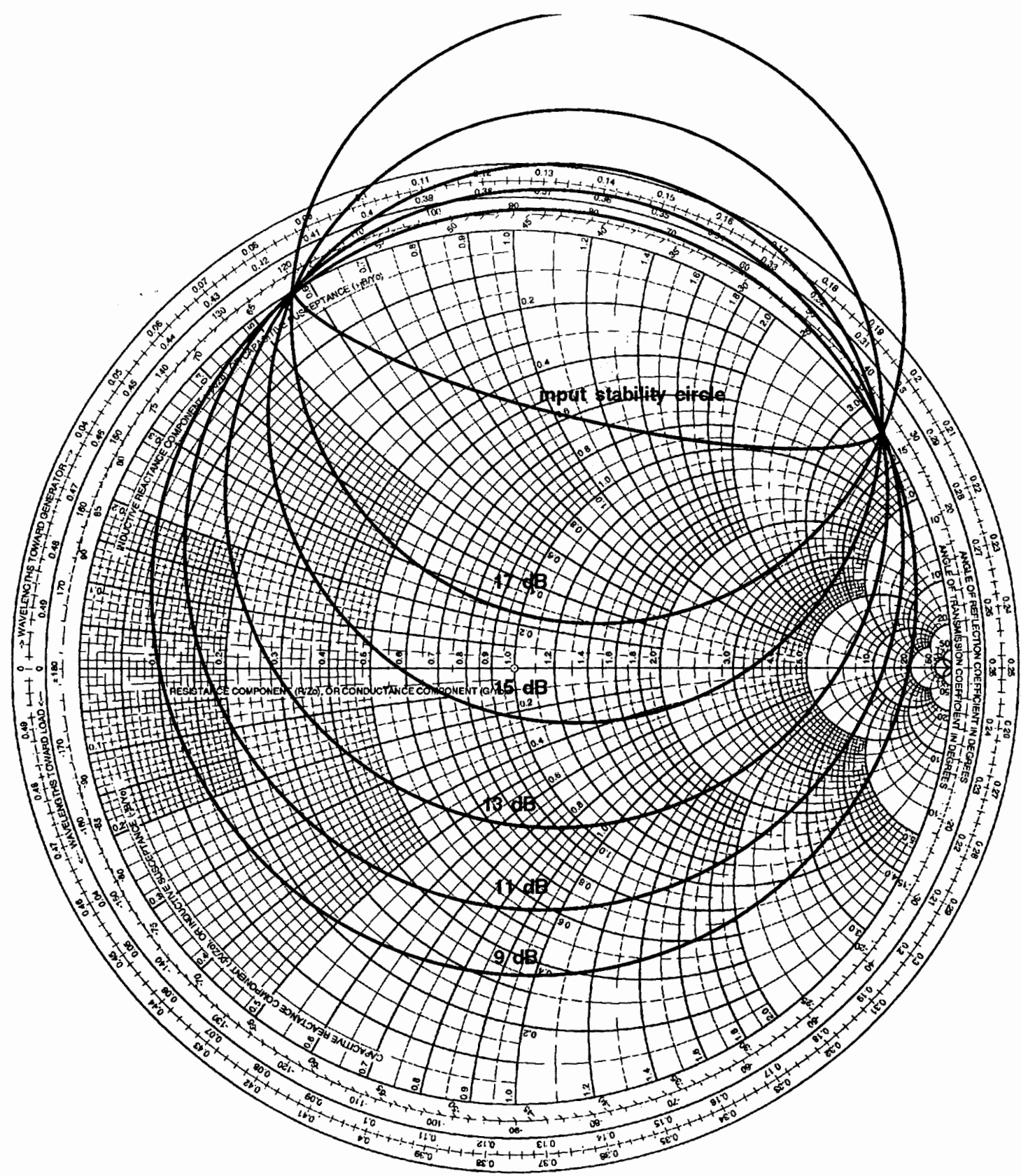

Figure 30. Gain and stability circles for N16 at frequency $=900 \mathrm{MHz}$. 


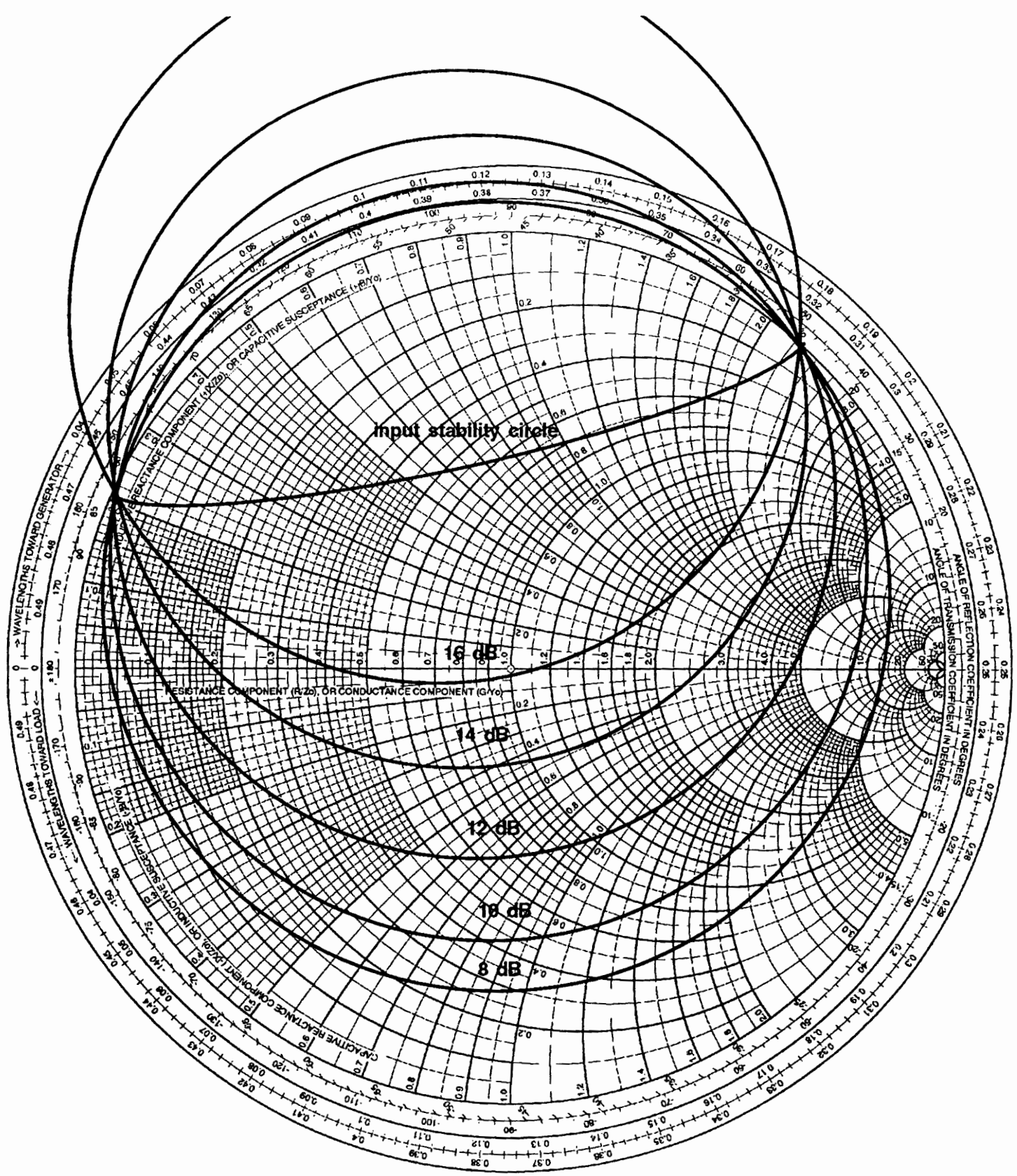

Figure 31. Gain and stability circles for G14V102 at frequency $=900 \mathrm{MHz}$. 


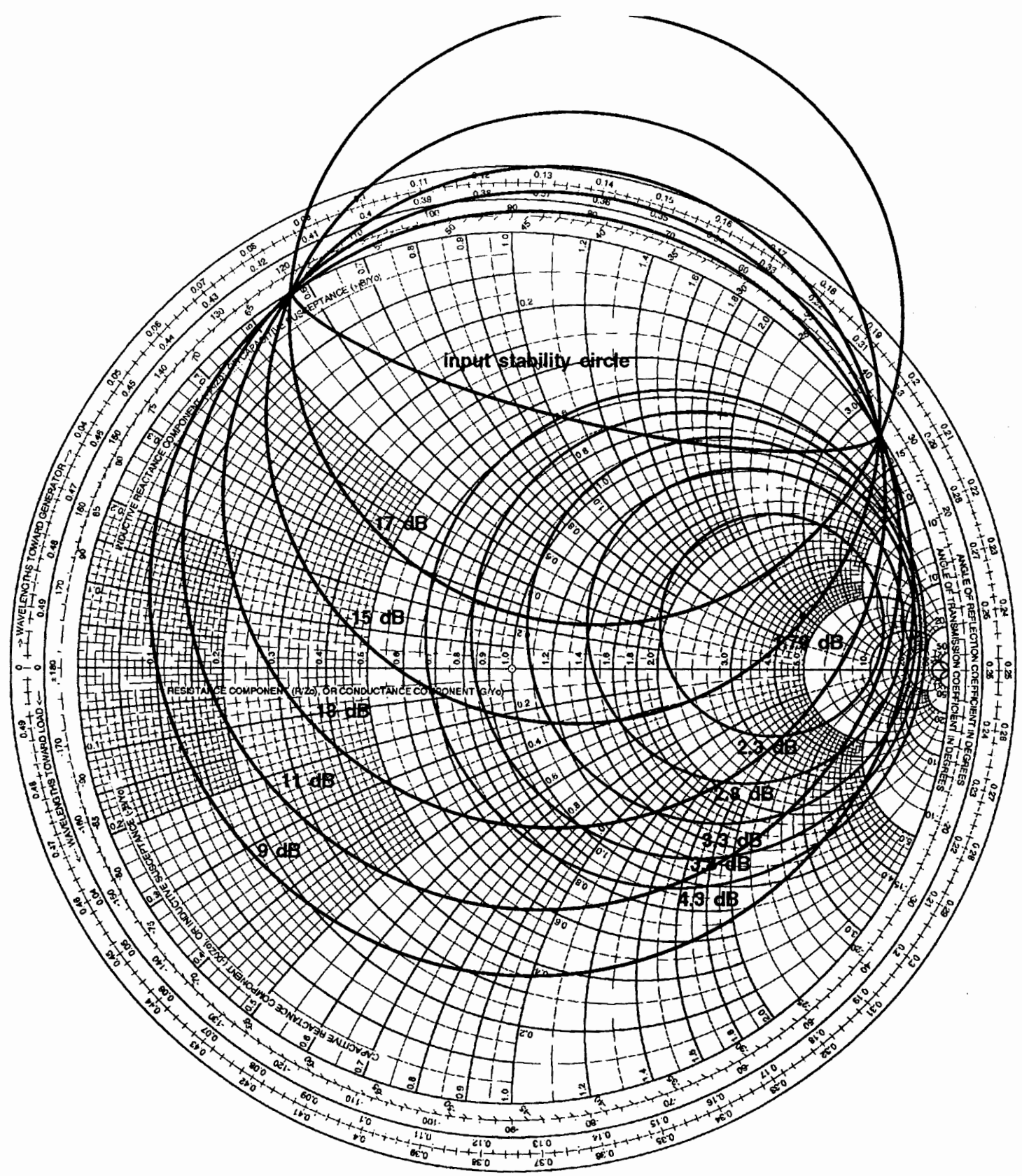

Figure 32. Gain and noise figure circles for N16 at frequency $=900 \mathrm{MHz}$. 


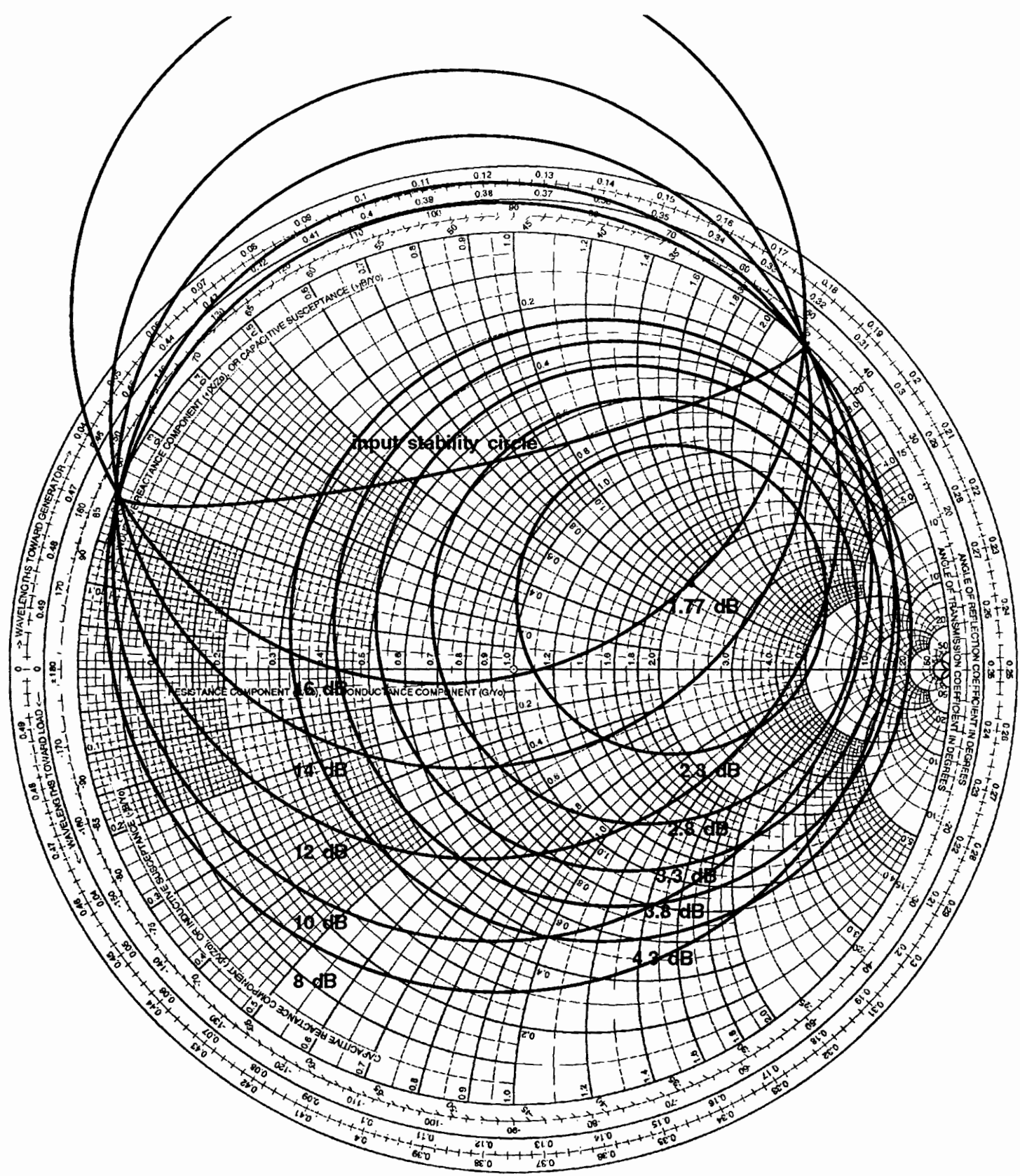

Figure 33. Gain and noise figure circles for G14V102 at frequency $=900 \mathrm{MHz}$. 


\section{CHAPTER V}

\section{CONCLUSION}

Noise is created by many physical processes which cannot be avoided. Living with noise means we must be able to measure and predict it. The noise sources in a bipolar transistor have been identified. At microwave frequencies they are thermal noise due to base resistance and shot noise from the base and collector currents. The base resistance consists of two parts. The external base resistance, $R_{b 1}$, is the resistance of the path between the base contact and the edge of the emitter diffusion. The active base resistance, $R_{b 2}$, is that resistance between the edge of the emitter and the site within the base region at which the current is actually flowing. $R_{b 1}$ can be reduced by decreasing the separation between the base and the emitter. This method is straightforward, but it is technology-limited. While the effect of current crowding in the base at high current level will reduce the effect of $R_{b 2}$, but more shot noise will be generated by the higher current.

The technique of measuring noise parameters $\left(F_{\min }, R_{n}, G_{o}\right.$, and $\left.B_{o}\right)$ of a bipolar transistor has been presented. This same measurement technique can also be employed to measure the noise parameters of a general two-port network.

This measurement technique can still be improved. The least-square-fit method is applied to measure $F$ only. The same method will also need to be applied to the measurement of $G_{o}$, and $B_{o}$. All the hardware and software need to be integrated together. A computer driven tuner will be needed to vary the source impedance for each noise measurement. 


\section{REFERENCE}

[1] "S-parameter Design,” Hewlett-Packard Application Note 154, Mar. 1990.

[2] Guillermo Gonzalez, Microwave Transistor Amplifiers, Prentice-Hall, New Jersey, 1984.

[3] P. R. Gray and R. G. Meyer, Analysis and Design of Analog Integrated Circuits, 2nd ed., John Wiley \& Sons, New York, 1984.

[4] Z. Y. Chang and M. C. Willy, Low-Noise Wide-Band Amplifiers in Bipolar and CMOS Technologies, Kluwer Academic Publishers, Norwell, MA, 1991.

[5] Harry F. Cooke, "Transistor Noise Figure," Solid State Design, pp. 37-42, Feb. 1963.

[6] H. A. Haus et al., "IRE Standards on Methods of Measuring Noise in Linear Twoports, 1959," Proc. IRE, vol. 48, pp. 60-68, Jan. 1960.

[7] H. A. Haus et al., "Representation of Noise in Linear Twoports," Proc. IRE, vol. 48, pp. 69-74, Jan. 1960.

[8] W. Kokyczka, A. Leupp, and M. J. O. Strutt, "Computer-aided determination of two-port noise parameters (CADON)," Proc. IEEE, vol. 58, pp. 1850-1851, Nov. 1970.

[9] Richard Q. Lane, "The Determination of Device Noise Parameters," Proc. IEEE, vol. 57, pp.1461-1462, Aug. 1969.

[10] G. Garuso and M. Sannino, "Computer-Aided Determination of Microwave Two-port Noise Parameters." IEEE Transactions on Microwave Theory and Techniques, vol. 26, pp. 639--642, Sept. 1978.

[11] H. Fukui, "The Noise Performance of Microwave Transistors," IEEE Transactions on Electron Devices, vol. 13, pp. 329-341, Mar. 1966.

[12] William Vetterling, Numerical Recipes Example Book (Pascal), Cambridge University Press, New York, 1985.

[13] K. Kurokawa, "Power Waves and the Scattering Matrix," IEEE Transactions on Microwave Theory and Techniques, vol. 13, pp. 194-202, Mar. 1965. 
[14] G. E. Bodway, “Two Port Power Flow Analysis Using Generalized Scattering Parameters," Microwave Journal, May 1967.

[15] D. Woods, "Reappraisal of the Unconditional Stability Criteria for Active 2-Port Networks in Terms of S Parameters," IEEE Transactions on Circuits and Systems, Feb. 1976.

[16] Ronald W. Kruse, "Microwave Design Using Standard Spice," Microwave Journal, pp. 164-171, Nov. 1988.

[17] W. Baechtold and M. J. O. Strutt, "Noise in Microwave Transistors," IEEE Transactions on Microwave Theory and Techniques, vol. 16, Sept. 1968.

[18] H. T. Friis, "Noise Figure of Radio Receivers," Proc. IRE, vol. 32, pp. 419-422, July 1944.

[19] - Richard Q. Lane, "A Microwave Noise and Gain Parameter Test Set," IEEE International Solid-State Circuits Conference, pp. 172-173, 1978.

[20] Neal Silence, "The Smith Chart and Its Usage in RF Design," RF Expo West Proceedings, pp. 1-7, Mar. 1992.

[21] George D. Vendelin and William C. Mueller, "Noise Parameters of Microwave Transistors," Microwave Journal, pp. 177-186, Nov. 1987.

[22] P. H. Smith, "Transmission Line Calculator," Electronics, vol. 12, pp. 29-31, Jan. 1939. 
APPENDIX A

CONSTRUCTION AND APPLICATION OF SMITH CHART 


\section{APPENDIX A \\ CONSTRUCTION AND APPLICATION OF SMITH CHART}

Equations like the one for reflection coefficient, $\Gamma=\frac{(\bar{Z}-1)}{(\bar{Z}+1)}$ are often used in microwave theory. Since all the values in this equation are complex numbers, the computations to solve this equation are tedious and "boring". Back in the thirties, Phillip Smith, a Bell Lab engineer, developed a graphical method for solving this often repeated equation [22]. In honor of his contribution, the chart is named Smith chart.

\section{CONSTRUCTION OF SMITH CHART}

The chart is essentially a mapping between two planes--the $Z$ or impedance plane and the $\Gamma$ or reflection coefficient plane. The actual mechanics of the transformation are accomplished as follows. Represent impedance $Z$ in rectangular format:

$$
Z=r+j x
$$

Divide the equation for reflection coefficient into its real and imaginary parts:

$$
\Gamma=u+j v=\frac{(r-1)+j x}{(r+1)+j x}
$$

Then separate the real and imaginary parts to obtain

$$
\begin{aligned}
& u=\frac{r^{2}-1+x^{2}}{(r+1)^{2}+x^{2}} \\
& v=\frac{2 x}{(r+1)^{2}+x^{2}}
\end{aligned}
$$

Eliminating $x$ from these equations yields

$$
\left(u-\frac{r}{r+1}\right)^{2}+v^{2}=\left(\frac{1}{r+1}\right)^{2}
$$


This equation represents a circle of radius $\frac{1}{(r+1)}$ with its center at $u=\frac{r}{(r+1)}$ and $v=0$. From this, a family of circles is obtained that represent a loci of constant resistance with their centers on the $u$ axis of Fig. 34 and a common point at $u=1$ and

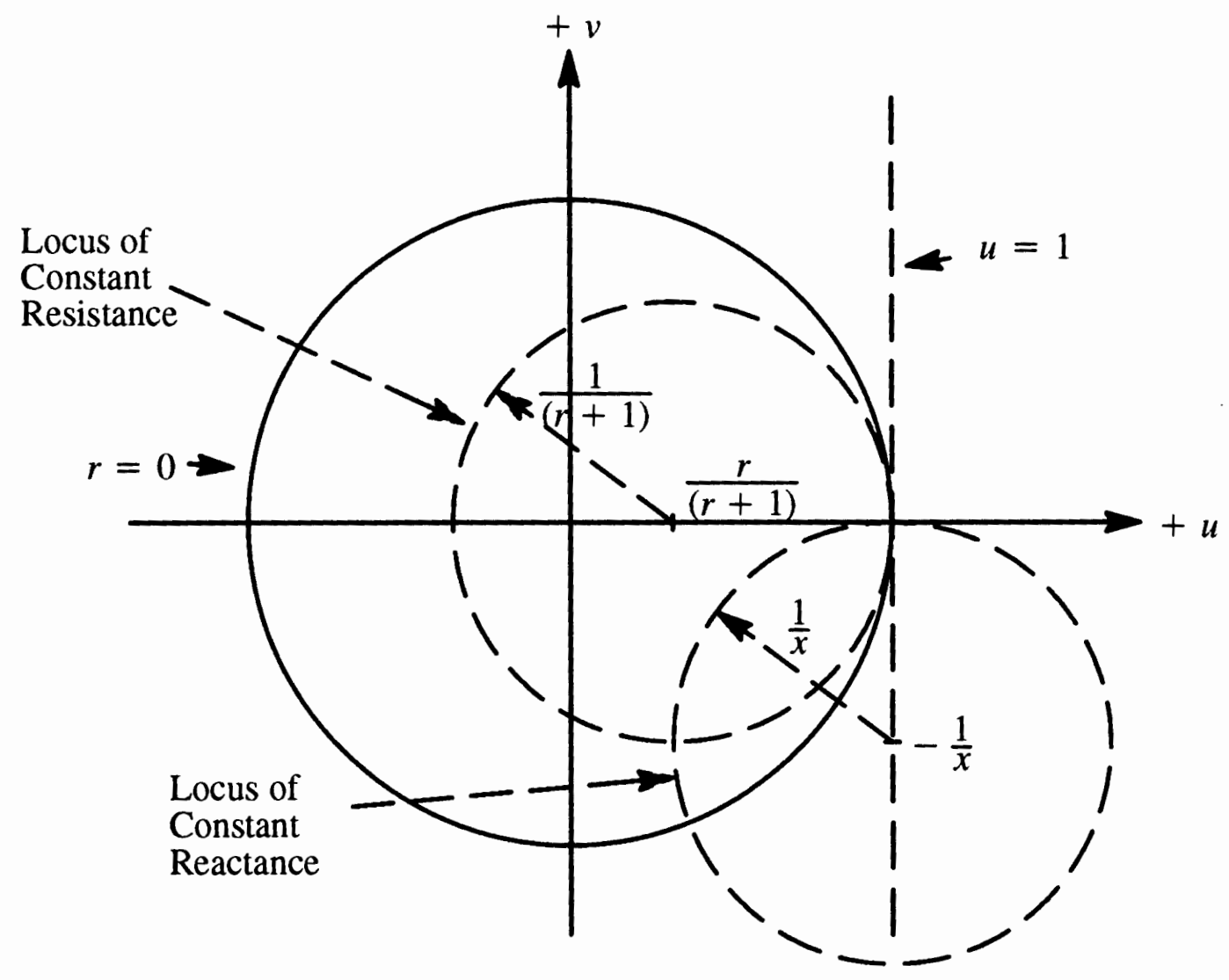

Figure 34. Smith chart construction.

$v=0$. For a range of $r$ from zero to infinity and $x=0$ all circles are contained within a circle described by a reflection coefficient of magnitude of 1 and variable phase, which is also the circle for $r=0$.

Eliminating $r$ from Eq. (85) and Eq. (86) yield the loci of constant reactance:

$$
(u-1)^{2}+\left(v-\frac{1}{x}\right)^{2}=\frac{1}{x^{2}}
$$

Again, a family of circles is obtained. However, this time the centers lie along a vertical 
line at points of $u=1$ and $v= \pm \frac{1}{x}$. Since the radii of there circles are $\frac{1}{x}$, they also have a common point of $u=1$ and $v=0$.

\section{APPLICATION OF SMITH CHART}

A point on a Smith chart simultaneously represents three different things.

Depending on the coordinate system used as a reference, they are: reflection coefficient, impedance, and admittance. For a system with input impedance of $Z_{\text {in }}=100+50 j \Omega$ in a $50 \Omega$ environment, the input reflection coefficient and the input admittance can be directly read from the chart.

Normalize $Z_{\text {in }}$ to $50 \Omega$ to get $\bar{Z}_{\text {in }}=2+1 j$, and locate this point on the Smith chart, point A. Draw a line from the center of the chart passed point $\mathrm{A}$ and intercept with the peripheral dial. The distance between the center of the chart and point $\mathrm{A}$ is the magnitude of the reflection coefficient and the angle indicated on the dial is the phase. For this case $\Gamma_{\text {in }}=0.45 \angle 26.6^{\circ}$. By looking at the admittance circles, the input admittance is $\bar{Y}_{i n}=0.4-0.2 j$.

The application of Smith is not limited to obtain the reflection coefficients of a circuit. It can be used as a graphical aid for impedance transformation, to show the variation of $\bar{Z}$ and $\bar{Y}$ with frequency, to determine the standing wave patterns, et al. For a detail explanation on the usage of the Smith chart, "Microwave Transistor Amplifiers," by Gonzalez [2] is one of the many good references to consult with. 


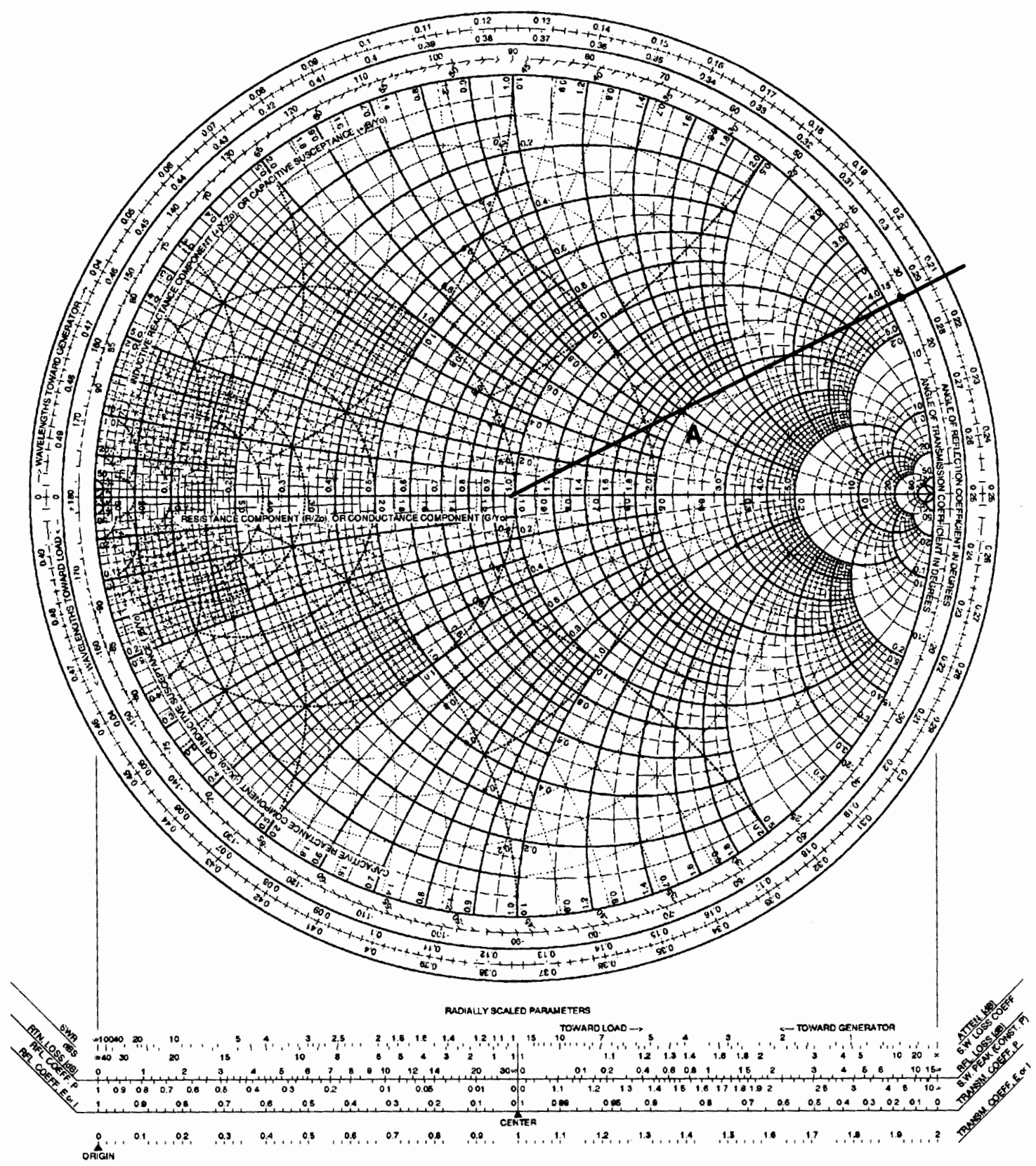

Figure 35. A complete Smith chart. 


\section{APPENDIX B}

NOISE FIGURE CALCULATIONS 


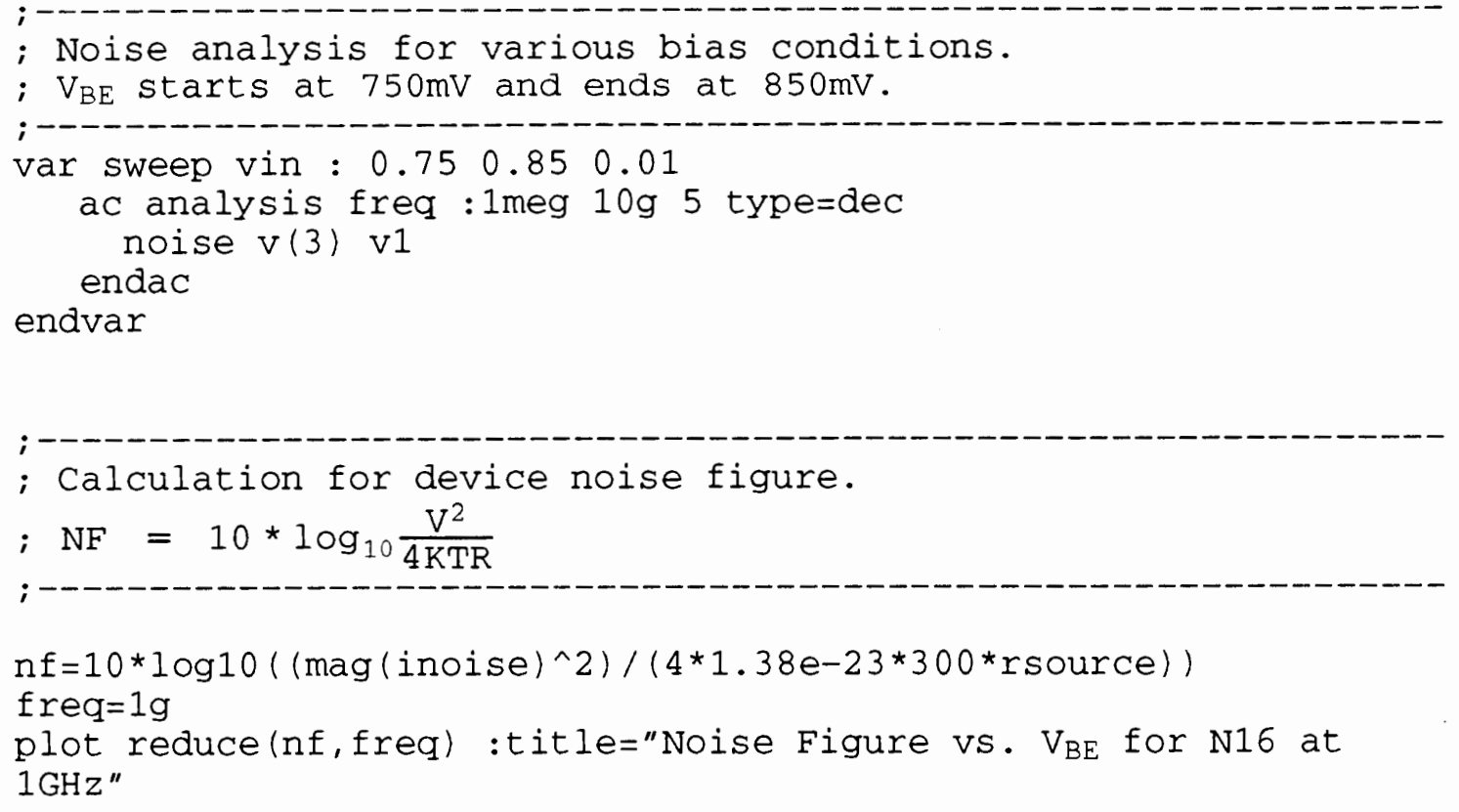


APPENDIX C

NOISE PARAMETERS AND NOISE FIGURE CIRCLES

CALCULATIONS 


\section{APPENDIX C \\ NOISE PARAMETERS AND NOISE FIGURE CIRCLES}

\section{CALCULATIONS}

This Pascal program uses the least-square-fit method and the square matrix inversion subroutine from "Numerical Recipes Example Book" [12] to calculate noise parameters $\left(F_{o}, R_{n}, B_{o}\right.$, and $G_{o}$ ) from noise figure measurements.

The input file contains the real part and imaginary part of the source impedance and the noise figure measured for that source impedance. It is in the form of $R_{s}, X_{s}, N F$. The program is written to accept a maximum of 25 sets of data.

$\begin{array}{lcl}140 & 50 & 2.004 \\ 50 & -17.5 & 3.641 \\ 37.5 & -10 & 4.210 \\ 20 & -26.25 & 6.157 \\ 70 & 70 & 2.594 \\ 25 & 35 & 4.836 \\ 16 & 10 & 6.396 \\ 70 & 5 & 2.861 \\ 60 & 15 & 3.045 \\ 40 & 12.5 & 3.856\end{array}$

The following is a sample output from the noise parameters calculation program. It consists of two sections. The first part gives the four noise parameters $N F_{\min }, R_{n}, G_{o}$, and $B_{o}$. The second part gives the coordinates of noise figure circles on a Smith chart in $0.5 d B$ increment from the minimum noise figure.

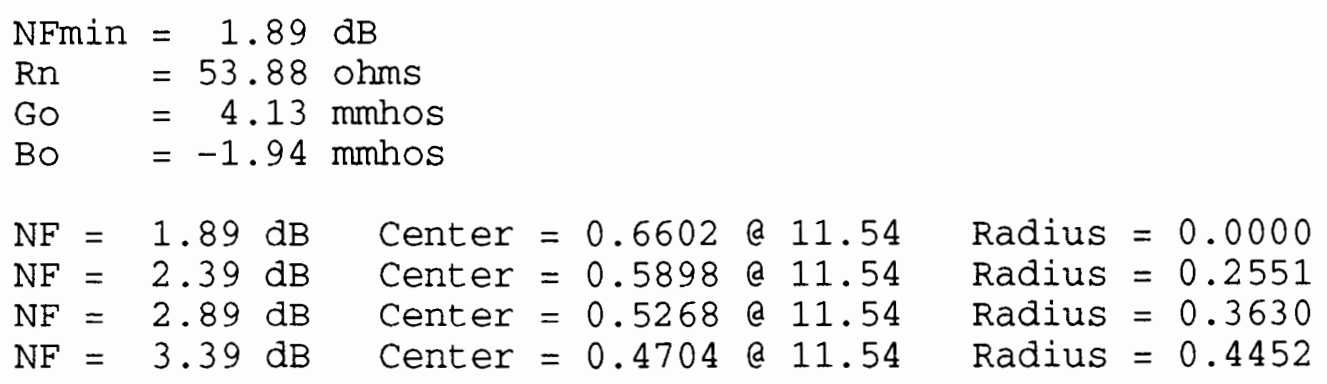




$\begin{array}{llll}\mathrm{NF}=3.89 \mathrm{~dB} & \text { Center }=0.4200 \text { @ } 11.54 & \text { Radius }=0.5128 \\ \mathrm{NF}=4.39 \mathrm{~dB} & \text { Center }=0.3749 \text { @ } 11.54 & \text { Radius }=0.5703 \\ \mathrm{NF}=4.89 \mathrm{~dB} & \text { Center }=0.3346 \text { @ } 11.54 & \text { Radius }=0.6199 \\ \mathrm{NF}=5.39 \mathrm{~dB} & \text { Center }=0.2986 \text { @ } 11.54 & \text { Radius }=0.6632 \\ \mathrm{NF}=5.89 \mathrm{~dB} & \text { Center }=0.2664 \text { @ } 11.54 & \text { Radius }=0.7011 \\ \mathrm{NF}=6.39 \mathrm{~dB} & \text { Center }=0.2376 \text { a } 11.54 & \text { Radius }=0.7346 \\ \mathrm{NF}=6.89 \mathrm{~dB} & \text { Center }=0.2120 \text { @ } 11.54 & \text { Radius }=0.7641\end{array}$

The followings are the block diagram and the complete listing of the Pascal program developed for calculating noise parameters for a two-port network.

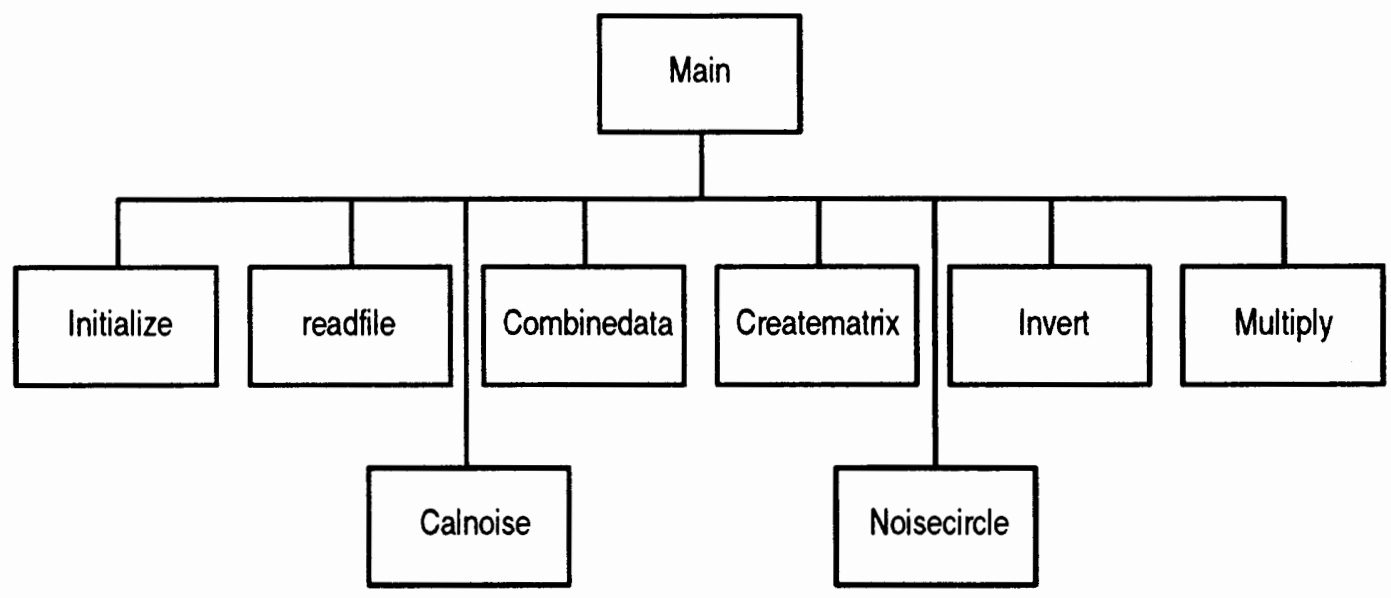

Figure 36. Flow chart for noise parameters calculation.

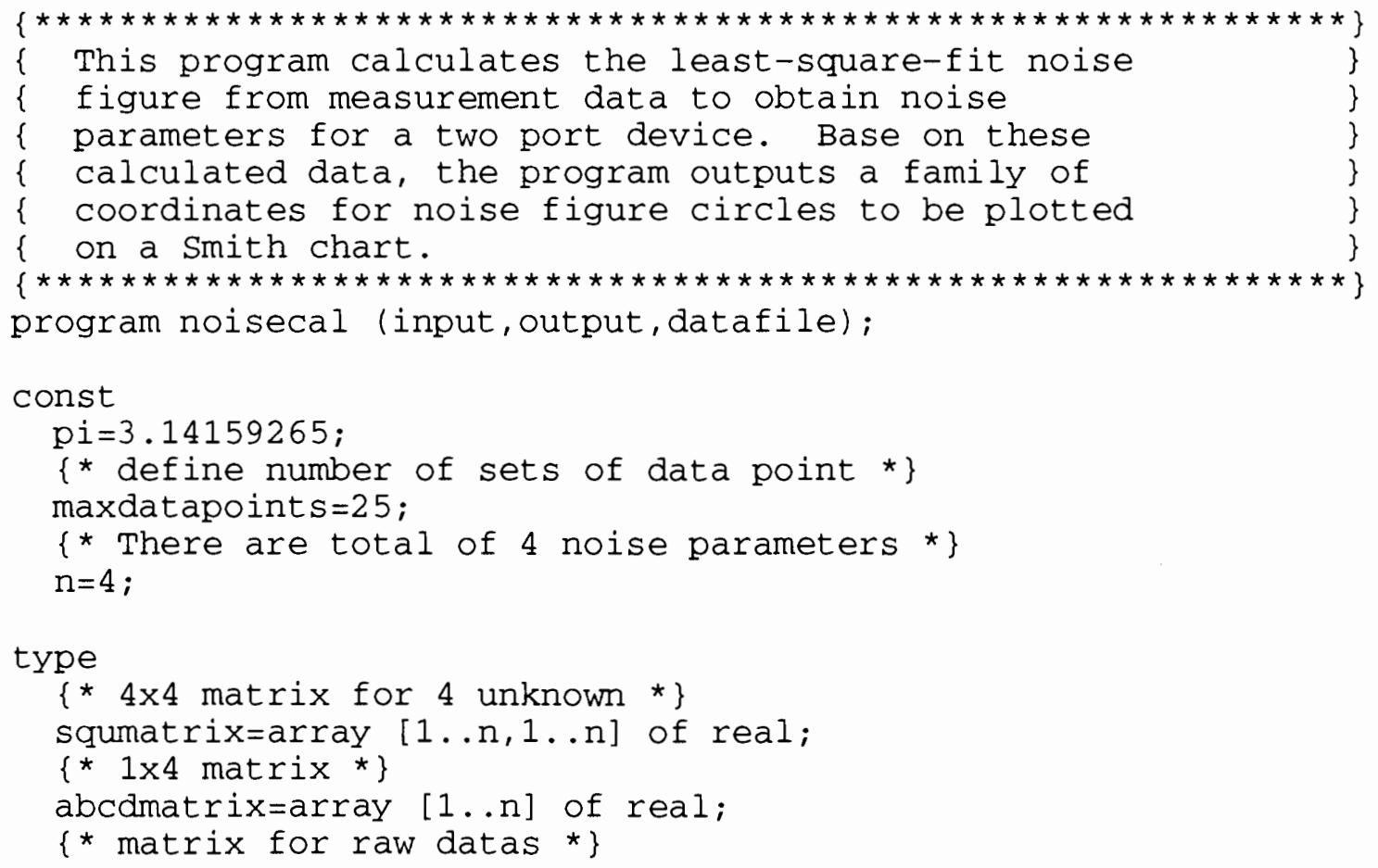


measurematrix=array [1..maxdatapoints] of real;

$\{$ * data points for NF circles *\}

circledata=array $[1 \ldots 10]$ of real;

$\operatorname{var}$

num, $i$ : integer;

fmin, rn, bopt, gopt, maggammao, phasegammao : real;

gs, bs, $f, x, y, z$ : measurematrix;

a, ai : squmatrix;

b, result : abcomatrix;

center, radius : circledata;

datafile : text;

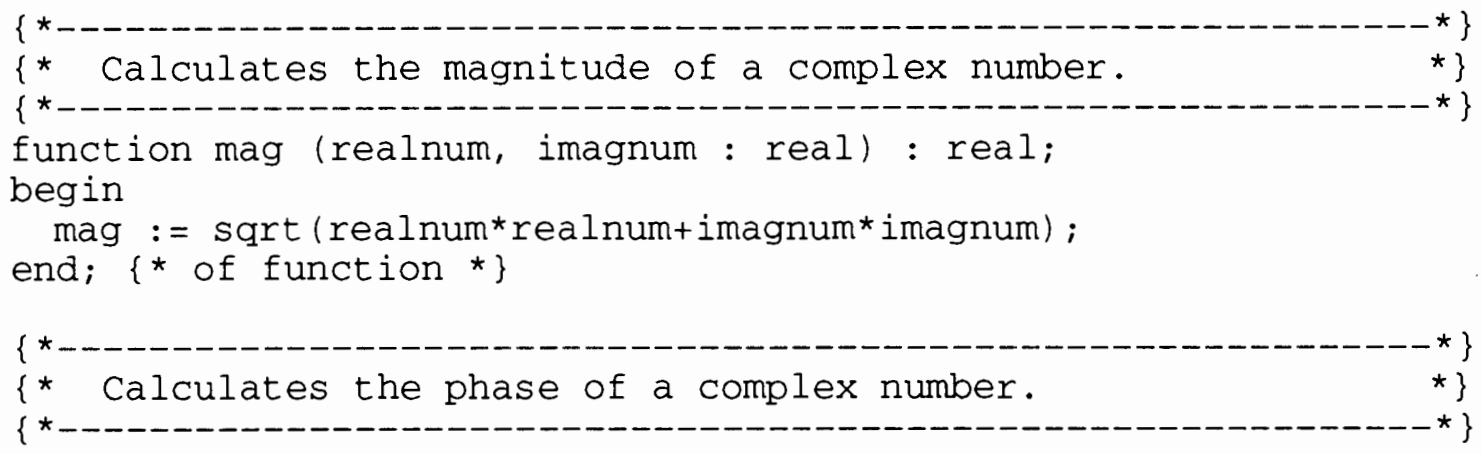

function phase (realnum, imagnum : real) : real;

var

temp : real;

begin

if (realnum $=0)$ and (imagnum $=0$ )

then

temp $:=0$;

if (realnum $=0$ ) and (imagnum $<>0$ )

then

if (imagnum $>0$ )

then

else

temp $:=\mathrm{pi} / 2$

temp $:=(-1) * \mathrm{p} i / 2$;

if (realnum $<>0$ ) and (imagnum $=0$ )

then

if $($ realnum $>0)$

then

temp $:=0$

else

temp := pi;

if (realnum $<>0$ ) and (imagnum $<>0$ )

then

begin

temp := arctan (imagnum/realnum);

if (realnum < 0 )

then

end;

$$
\text { temp := temp + pi; }
$$

phase := temp; 
end; $\{$ * of function * $\}$

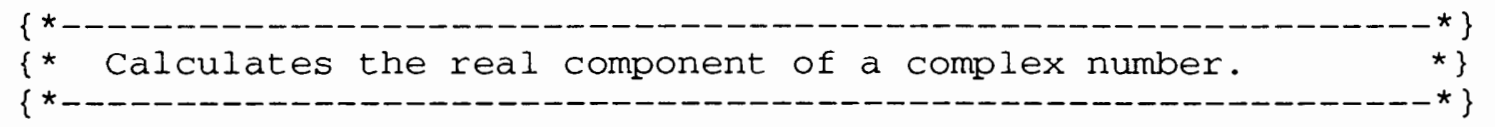

function realpart (magl, phasel : real) : real;

begin

realpart $:=\operatorname{mag} 1 * \cos ($ phasel);

end; $\{$ * of function *

$\{$ Calculates the imaginary part of a complex number.

function imagpart (magl, imagl :real) :real;

begin

imagpart := magl * $\sin (i \operatorname{mag} 1)$;

end; $\{$ * of function *

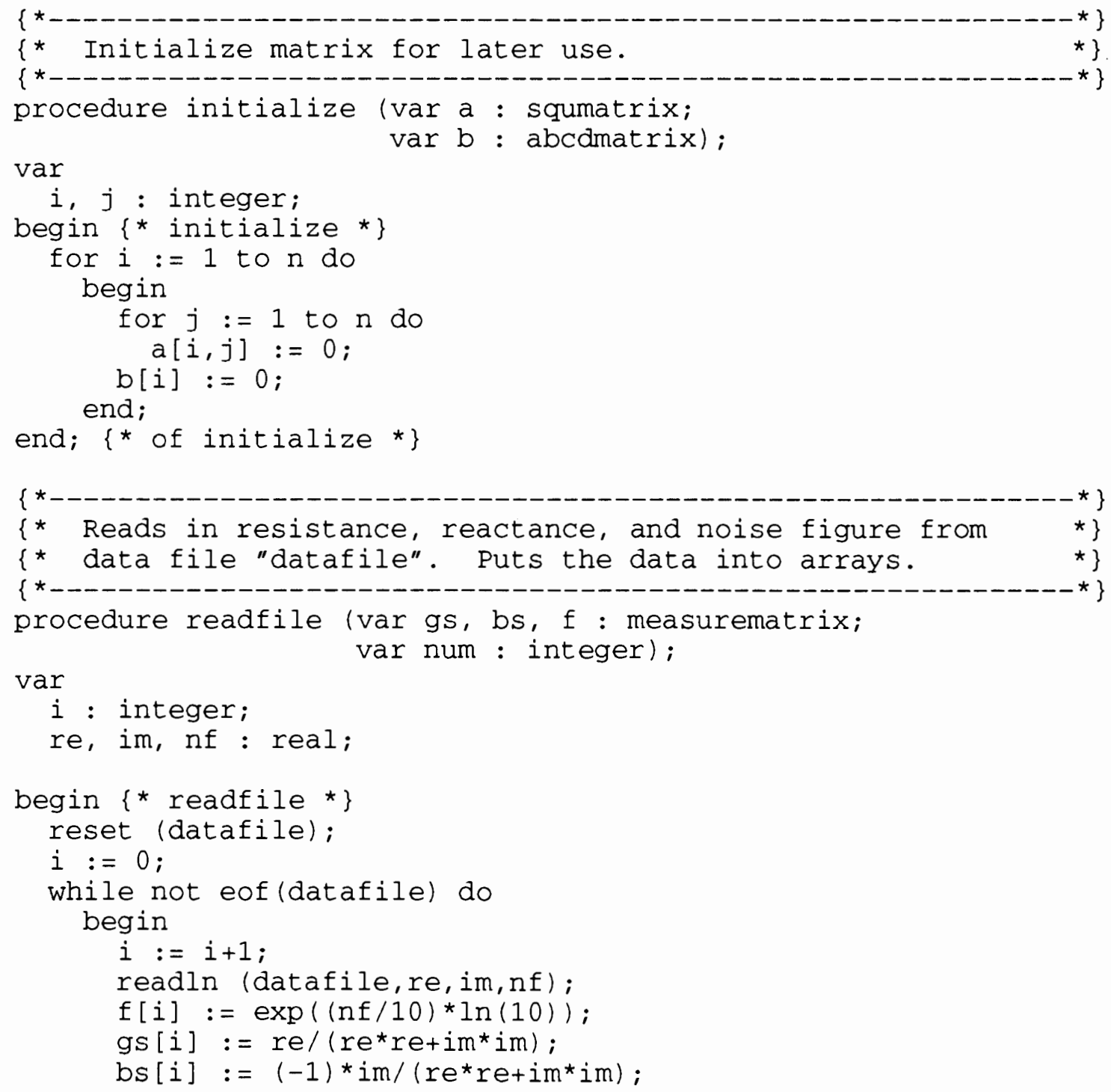


end;

num : $=i$;

end; $\{$ of readfile * $\}$

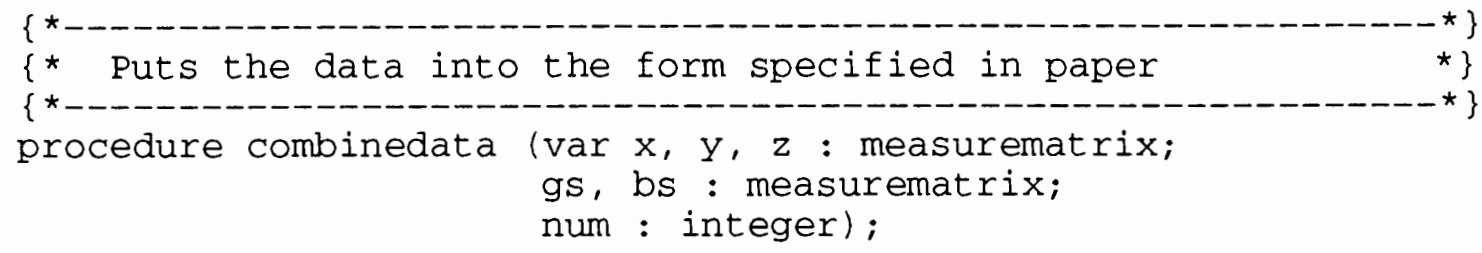

var

i : integer;

begin $\{$ * combinedata *

for $i:=1$ to num do

begin

$x[i]:=g s[i]+(\operatorname{bs}[i] * b s[i]) / g s[i]$;

$y[i]:=1 / g s[i] ;$

$z[i]:=b s[i] / g s[i]$;

end;

end; $\{$ * of combinedata * $\}$

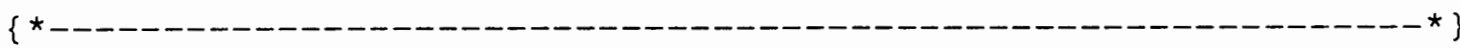

$\{$ * Creates the two matrixs to solve the four noise * $\}$

$\{$ * parameters. *

$\{$ « - - - - - - - - - - - - - - - - - - - - - - - - - - - - - - - - - - - - - - - - * $\}$

procedure creatematrix (x, $y, z$, $\mathrm{f}$ : measurematrix;

num : integer;

var a:squmatrix;

var b:abcdmatrix);

var

$i, j$ : integer;

begin $\{$ * creatematrix *

\{ Calculating a matrix \}

a $[1,1]:=$ num;

for $j:=1$ to num do

begin

$$
\begin{aligned}
& a[2,1]:=x[j]+a[2,1] ; \\
& a[3,1]:=y[j]+a[3,1] ; \\
& a[4,1]:=z[j]+a[4,1] ; \\
& a[2,2]:=x[j] \star x[j]+a[2,2] ; \\
& a[3,3]:=y[j] \star y[j]+a[3,3] ; \\
& a[4,4]:=z[j] * z[j]+a[4,4] ; \\
& a[3,2]:=x[j] \star y[j]+a[3,2] ; \\
& a[4,2]:=x[j] * z[j]+a[4,2] ; \\
& a[3,4]:=y[j] * z[j]+a[3,4] ;
\end{aligned}
$$

end;

for $i:=2$ to 4 do

$\mathrm{a}[1, i]:=\mathrm{a}[\mathrm{i}, 1]$;

$\mathrm{a}[2,3]:=\mathrm{a}[3,2]$;

$\mathrm{a}[2,4]:=\mathrm{a}[4,2]$; 
$a[4,3]:=a[3,4]$;

\{Calculating b matrix \}

for $j:=1$ to num do

begin

$\mathrm{b}[1]:=f[j]+b[1] ;$

$b[2]:=x[j] * f[j]+b[2] ;$

$b[3]:=y[j] * f[j]+b[3] ;$

$b[4]:=z[j] * f[j]+b[4] ;$

end; $\{$ * creatematrix * $\}$

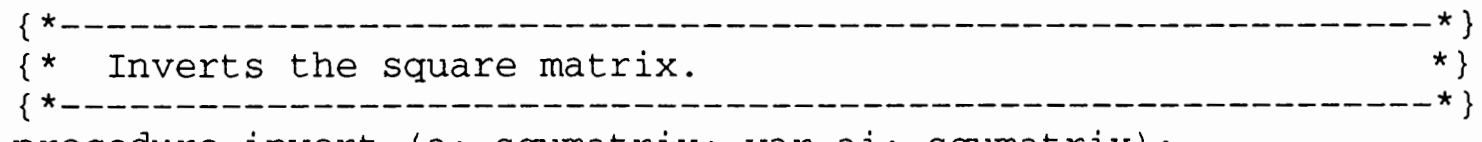

procedure invert (a: squmatrix; var ai: squmatrix);

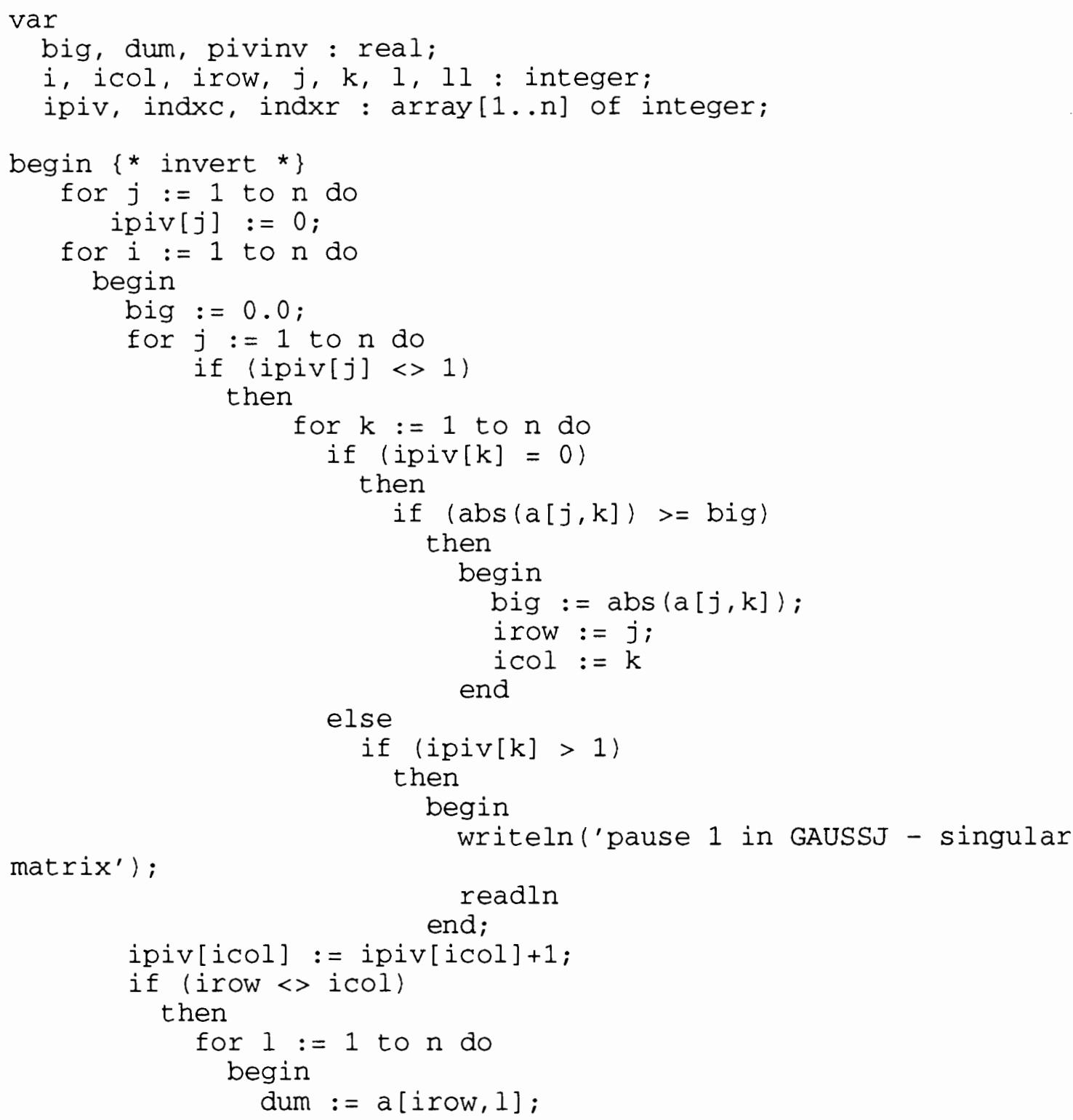




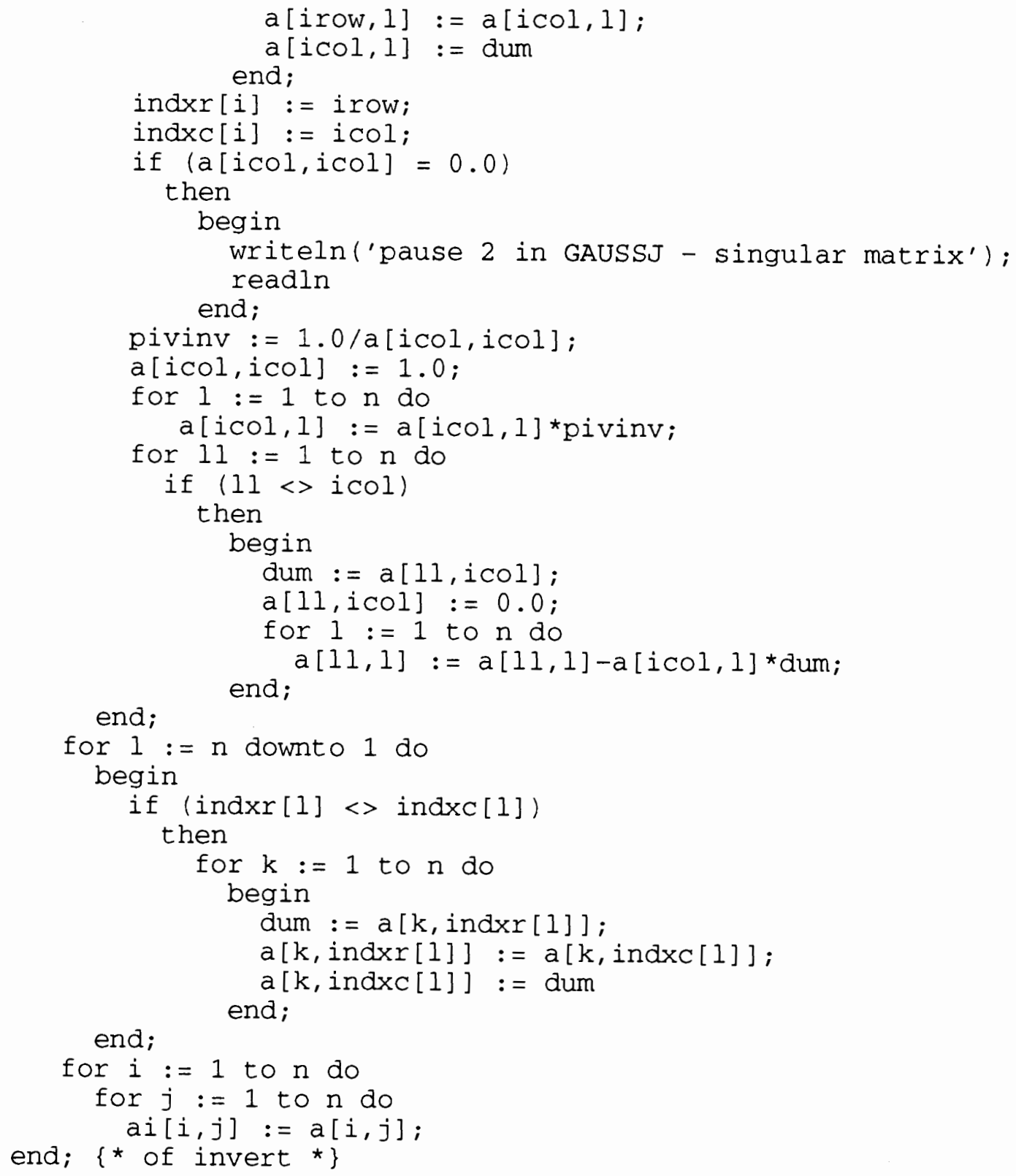




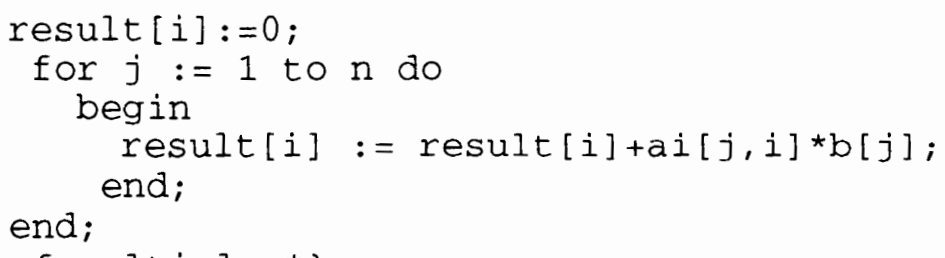

end; $\{$ * of multiply * $\}$

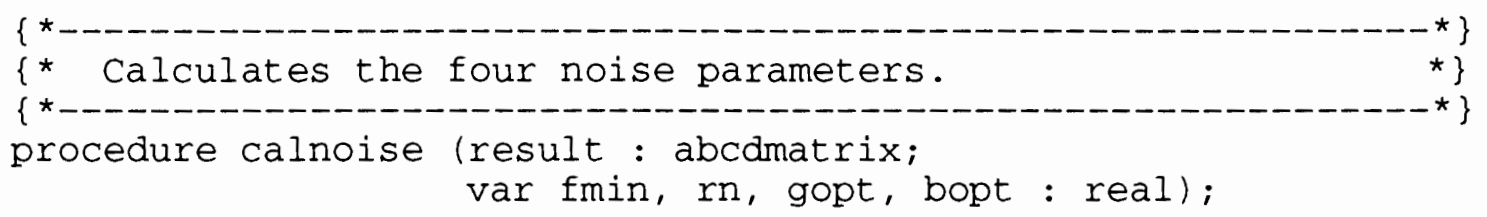

var

$$
a, b, c, d \text { : real; }
$$

begin $\{$ *alnoise * $\}$

$a:=\operatorname{result}[1]$;

$\mathrm{b}:=$ result $[2]$;

$c:=$ result $[3] ;$

$\mathrm{d}:=\operatorname{result}[4]$;

fmin $:=a+\operatorname{sqrt}\left(4 * b * c-d^{*} d\right)$;

if fmin $<=0$ then

begin

writeln ('fmin $<=0$; take more data point'); readln;

end;

rn $:=b$;

gopt $:=(\operatorname{sqrt}(4 * b * c-d * d)) /(2 * b)$;

bopt $:=(-1) * d /(2 * b)$;

end; $\{$ * of calnoise * $\}$

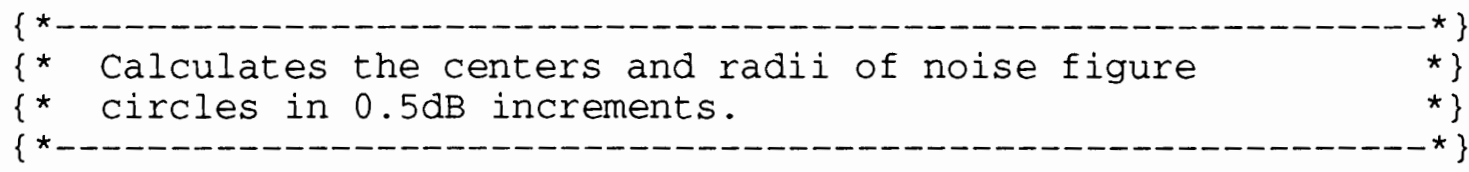

procedure noisecircle (fmin, rn, maggammao, phasegammao : real;

var center, radius : circledata);

var

$i$ : integer;

temp, ni, reg, img : real;

begin

reg := realpart (maggammao, phasegammao);

img := imagpart (maggammao, phasegammao);

temp := mag $(1+r e g, i m g) * m a g(1+r e g, i m g)$;

for $i:=1$ to 10 do

begin

ni $:=(\operatorname{fmin} *(\exp (0.05 * i * \ln (10))-1)) /(4 * r n / 50) * \operatorname{temp} ;$

center $[\mathrm{i}]:=$ maggammao $/(1+\mathrm{ni})$; radius $[i]:=$

$1 /(1+n i) * \operatorname{sqrt}(n i * n i+n i *(1-$ maggammao*maggammao $))$; 
end;

end; $\{$ * of noisecircle * $\}$

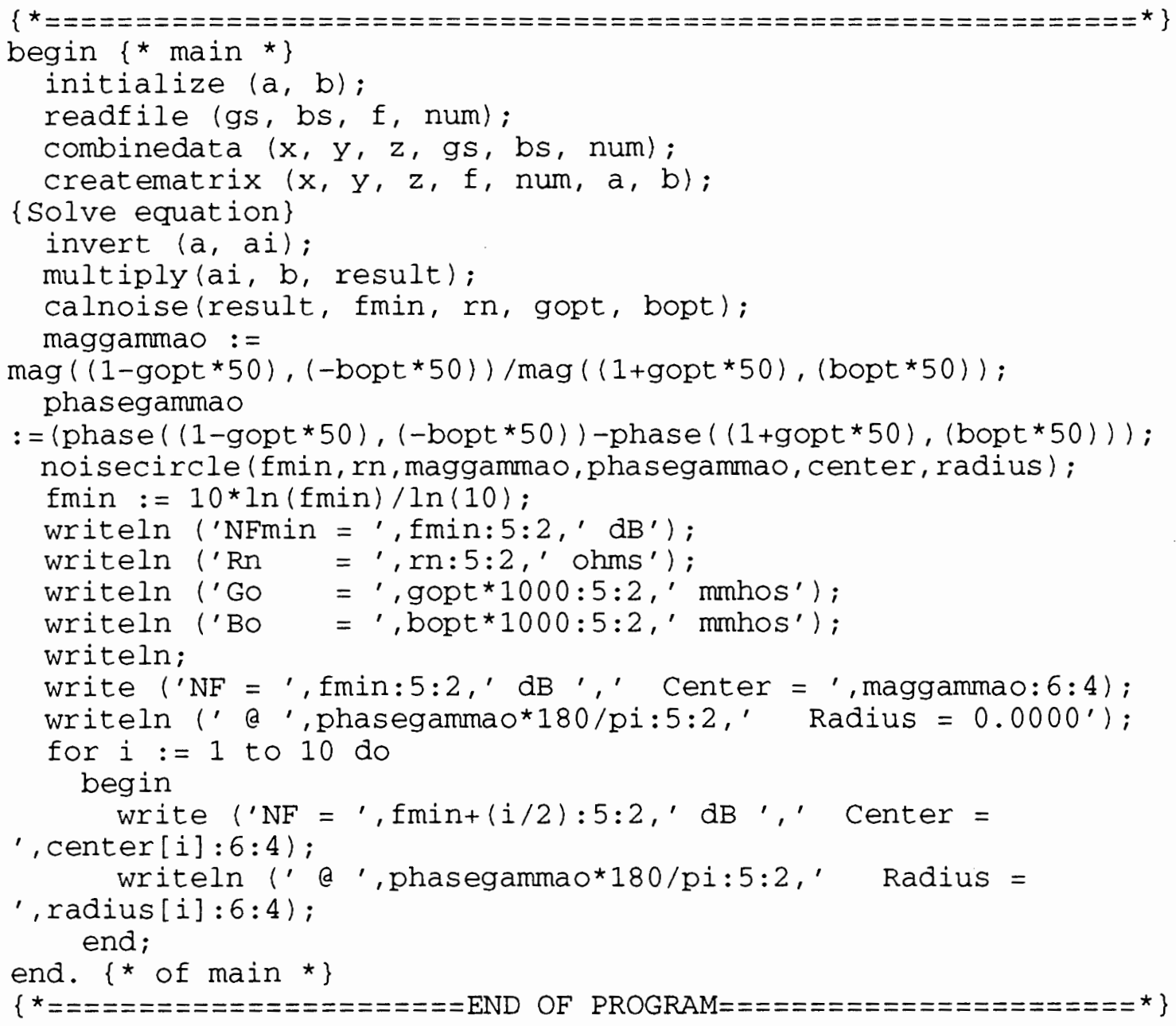




\section{APPENDIX D}

STABILITY CIRCLES COMPUTATIONS 


\section{APPENDIX D \\ STABILITY CIRCLES COMPUTATIONS}

\section{Stability Criteria}

This TEKSPICE script calculates and prints out the two stability criteria, Eq. (89) and Eq. (90), from s-parameters of a two-port network.

$$
\begin{gathered}
|\Delta|=\left|S_{11} S_{22}-S_{12} S_{21}\right|<1 \\
K=\frac{1-\left|S_{11}\right|^{2}-\left|S_{22}\right|^{2}+|\Delta|^{2}}{2\left|S_{12} S_{21}\right|}>1
\end{gathered}
$$

; Clears all variables.

clear

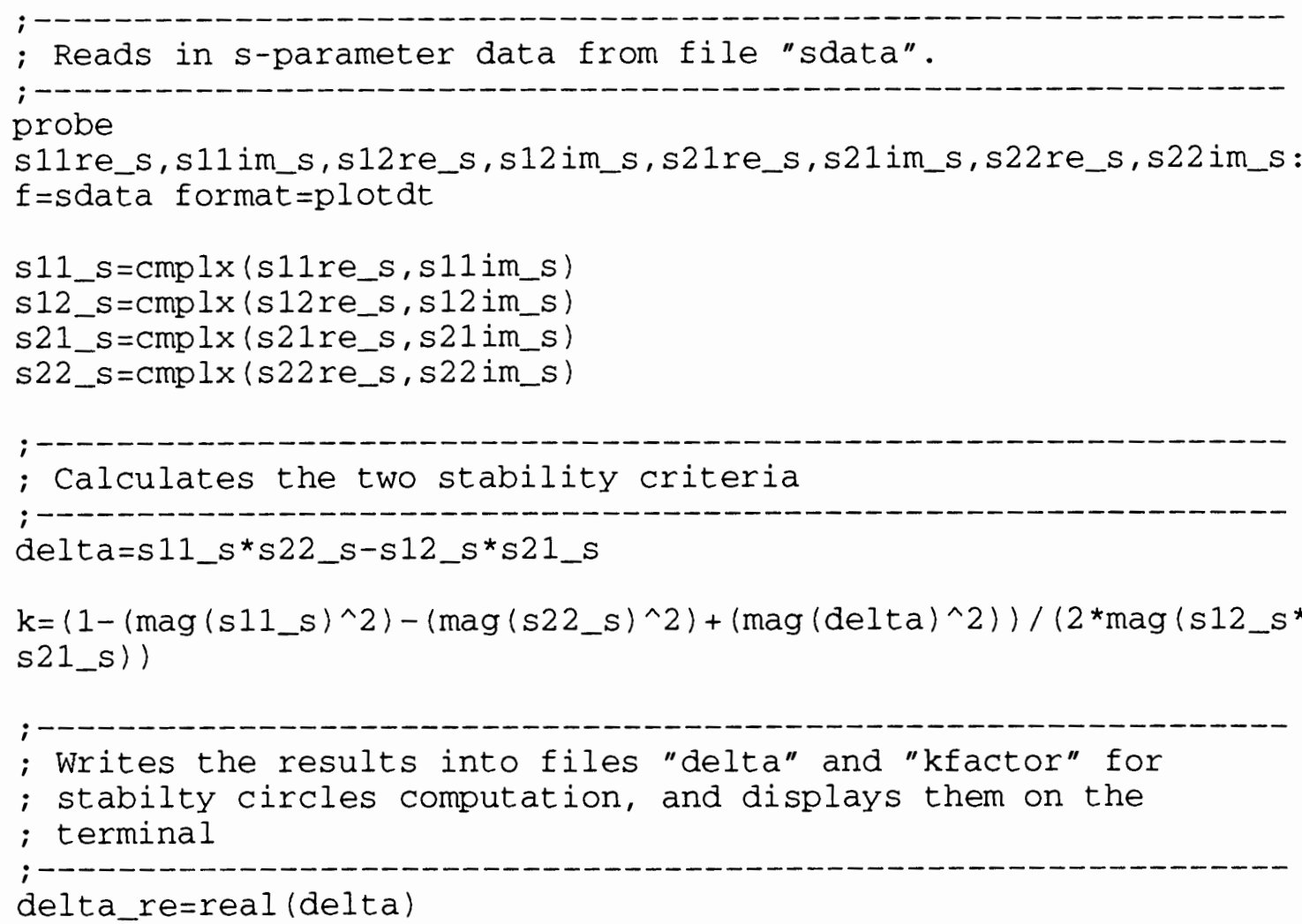


delta_im=imag (delta)

print delta_re, delta_im: $f=$ delta format=plotat

print $k: f=k f a c t o r$ format $=p l o t d t$

print $k$, mag (delta)

\section{Stability Circles Computation}

This TEKSPICE script calculates the input and output stability circles from s-parameters of a two-port network. The radius and center of the input stability circles are:

$$
\begin{aligned}
& r_{s}=\left|\frac{S_{12} S_{21}}{\left|S_{11}\right|^{2}-|\Delta|^{2}}\right| \\
& C_{s}=\frac{\left(S_{11}-\Delta S_{22}^{*}\right)^{*}}{\left|S_{11}\right|^{2}-|\Delta|^{2}}
\end{aligned}
$$

and the radius and center of the output stability circles are:

$$
\begin{aligned}
& r_{l}=\left|\frac{S_{12} S_{21}}{\left|S_{22}\right|^{2}-|\Delta|^{2}}\right| \\
& C_{l}=\frac{\left(S_{22}-\Delta S_{11}^{*}\right)^{*}}{\left|S_{22}\right|^{2}-|\Delta|^{2}}
\end{aligned}
$$

; Clears all variables.

clear

Reads in s-parameter data from file "sdata".

; - - - - - - - - - - - - - - - - - - - - - -

probe

s11re_s,s11im_s,s12re_s,s12im_s,s21re_s,s21im_s,s22re_s,s22im_s :

$\mathrm{f}=$ sdata format $=$ plotdt

s11_s=cmplx (s11re_s,s11im_s)

s12_s $=$ cmplx (s12re_s,s12im_s)

s21_s=cmplx (s21re_s,s21im_s)

s22_s =cmplx (s22re_s, s22im_s) 


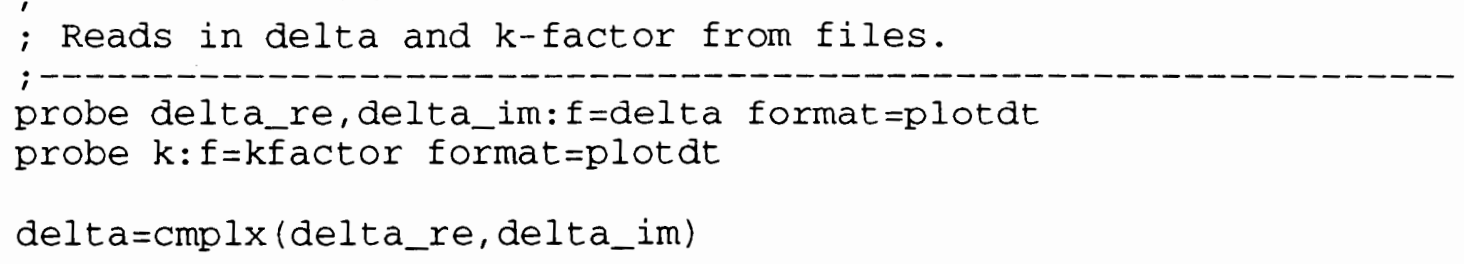

print

reduce (radius_out, freq), mag (reduce (center_out, freq)), phase (reduc e(center_out, freq)) 
APPENDIX E

GAIN CIRCLES COMPUTATION 


\section{APPENDIX E}

\section{GAIN CIRCLES COMPUTATION}

This TEKSPICE script calculates the center and radius of a gain circle from the s-parameters of a two-port network according to the following equations:

$$
\begin{gathered}
g_{a}=\frac{G_{A}}{\left|S_{21}\right|^{2}} \\
C_{1}=S_{11}-\Delta S_{22}^{*} \\
R_{a}=\frac{\sqrt{1-2 K\left|S_{12} S_{21}\right| g_{a}+\left|S_{12} S_{21}\right|^{2} g_{a}^{2}}}{\left|1+g_{a}\left(\left|S_{11}\right|^{2}-|\Delta|^{2}\right)\right|}
\end{gathered}
$$

and

$$
C_{a}=\frac{g_{a} C_{1}^{*}}{1+g_{a}\left(\left|S_{11}\right|^{2}-|\Delta|^{2}\right)}
$$

\footnotetext{
probe $\mathrm{f}=\mathrm{sdat} a$ format $=\mathrm{plot}$ lot

$$
\begin{aligned}
& \text { s11_s=cmp1x (s11re_s,s11im_s) } \\
& \text { s12_s }=\operatorname{cmplx} \text { (s12re_s, s12im_s) } \\
& \text { s21_s }=\text { cmplx (s21re_s,s21im_s) } \\
& \text { s22_s=cmplx (s22re_s,s22im_s) }
\end{aligned}
$$

; Reads in s-parameter data from file "sdata".

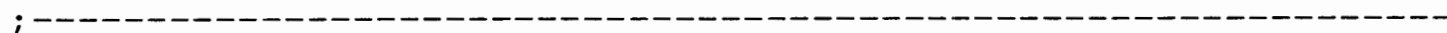

s11re_s,s11im_s,s12re_s,s12im_s,s21re_s,s21im_s,s22re_s,s22im_s:

; Reads in delta and $\mathrm{k}$-factor from files.

probe delta_re, delta_im:f=delta format=plotat

probe $k: f=k f a c t o r$ format $=p l o t d t$

delta=cmplx (delta_re,delta_im)

glinear $=10^{\wedge}(\mathrm{ga} / 10)$ 
gain=glinear $/\left(\operatorname{mag}\left(s 21 \_s\right) \wedge 2\right)$

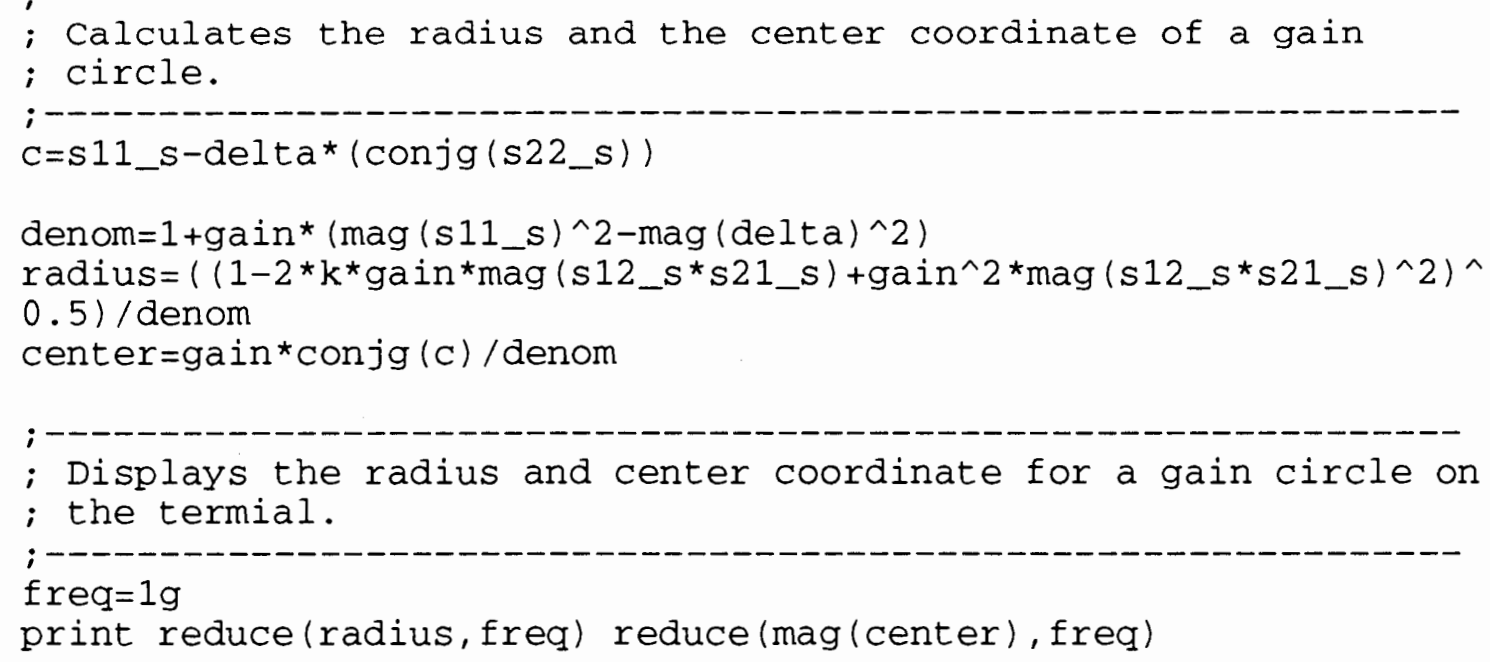




\section{APPENDIX F}

\section{S-PARAMETERS EXTRACTION}




\section{APPENDIX F}

\section{S-PARAMETERS EXTRACTION}

This TEKSPICE script provides the s-parameters for a two-port network. The calculation of s-parameters from node voltages is derived in the text. The valus of the source voltage in $\mathrm{AC}$ analysis is arbitrary with respect to s-parameter calculation. The calculation is simplified by using a $2 \mathrm{~V} \mathrm{AC}$ source.

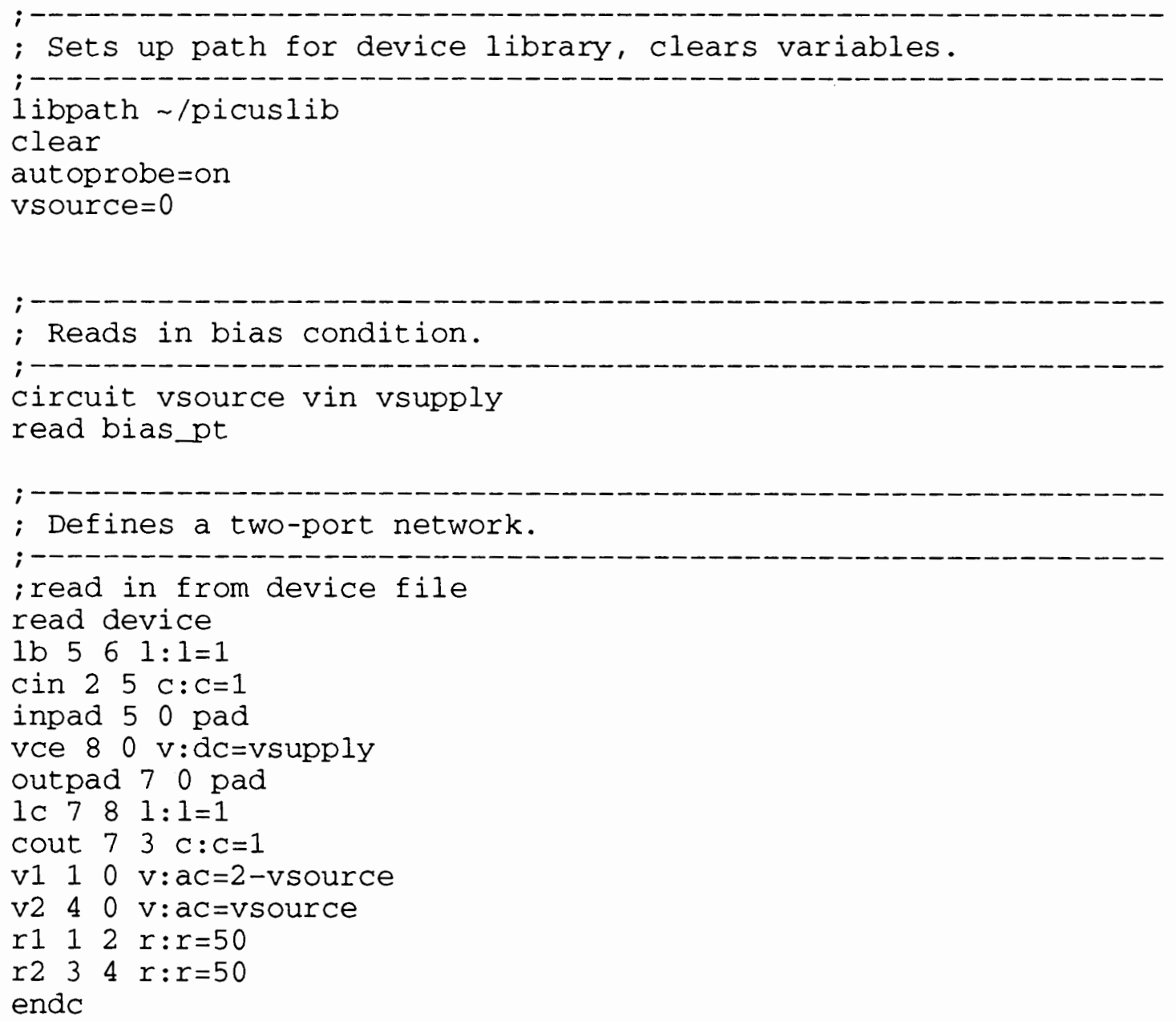




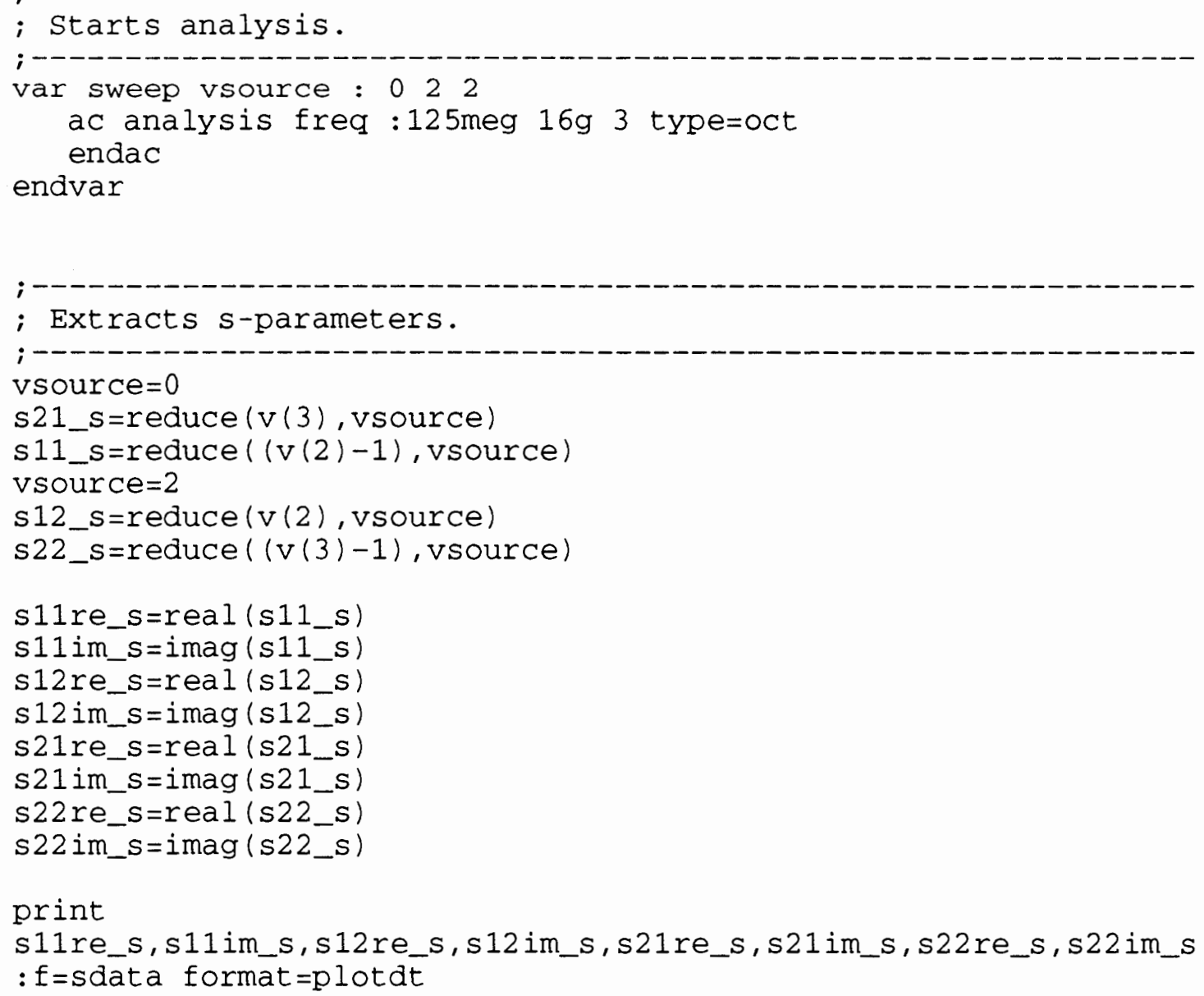

